\title{
16. RADIOLARIA FROM THE EASTERN NORTH PACIFIC, DEEP SEA DRILLING PROJECT, LEG 18
}

\author{
Stanley A. Kling, Cities Service Oil Company
}

$\begin{array}{lc} & \text { CONT } \\ & \text { Page } \\ & \\ \text { Introduction } & 617 \\ \text { Biostratigraphic Framework } & 618 \\ \text { Miocene } & 618 \\ \text { Pliocene } & 618 \\ \text { Pleistocene } & 618 \\ \text { Radiolarian Site Summaries } & 618 \\ \text { Site 172 } & 619 \\ \text { Site 173 } & 619 \\ \text { Site 174 } & 620 \\ \text { Site 175 } & 623 \\ \text { Site 176 } & 623 \\ \text { Site 177 } & 623 \\ \text { Site 178 } & 624 \\ \text { Site 179 } & 624 \\ \text { Site 180 } & 624 \\ \text { Site 181 } & 624 \\ \text { Site 182 } & 624 \\ \text { Radiolarian Events and Potential Datum Levels } & 624 \\ & \end{array}$

\section{INTRODUCTION}

Radiolarians occur at all the sites drilled on DSDP Leg 18, although two sites (172 and 174) produced only a few specimens in the surface sediment. Sites 173 and 178 produced sequences extending from Quaternary back into early Miocene, while sequences at other sites are of Pliocene-Pleistocene or Pleistocene age.

Site 173, with abundant well-preserved specimens throughout, provides the best known sequence of western North American Neogene radiolarians. Thus, it will be particularly important for establishing a radiolarian biostratigraphy for the eastern boundary current region where the equatorial zonation (Riedel and Sanfilippo, 1970, 1971; Moore, 1971) is not readily applicable. This will contribute to a better understanding of Miocene-Pliocene radiolarian sequences in nearby sections on land. Site 178 offers a potentially comparable sequence for the subarctic region, but poor preservation in much of the pre-Pleistocene section precludes a detailed comparison.

The methods of investigation and presentation of results follows closely those developed in reports for previous DSDP legs (Riedel and Sanfilippo, 1970, 1971; Riedel and Sanfilippo, in press; Kling, 1971). Because non-equatorial radiolarian assemblages have been studied relatively little, many of even the prominent species cannot yet be identified. Thus the synchronopticon (Plates 1-12), in which an effort is made to illustrate all prominent forms at
Page

$\begin{array}{ll}\text { Range Chart and Geographic Variation } & \\ \text { in Species Ranges } & 629 \\ \text { Evolutionary lineages } & 629 \\ \text { Preservation } & 633 \\ \text { Paleoecology } & 633 \\ \text { Possible Mesozoic (Franciscan) Occurrences } & 633 \\ \text { Systematics } & 633 \\ \text { Collosphaeridae } & 634 \\ \text { Actinommidae } & 634 \\ \text { Spongodiscidae } & 635 \\ \text { Litheliidae } & 635 \\ \text { Acanthodesmiidae } & 635 \\ \text { Theoperidae } & 635 \\ \text { Carpocaniidae } & 638 \\ \text { Pterocoryidae } & 638 \\ \text { Artostrobiidae } & 639 \\ \text { References } & 640 \\ \text { Plates } & 641\end{array}$

ten stratigraphic horizons at Site 173 , becomes particularly important.

Forms that could be identified with some confidence are tabulated for those sites in which radiolarians undergo observable stratigraphic change. The distinction among morphotypic, morphotypic-evolutionary, and evolutionary stratigraphic limits of species, suggested by Riedel and Sanfilippo (1971; Riedel and Sanfilippo, in press), is made in this study as nearly as possible. This concept has been useful in defining limits of a number of species with rather broadly overlapping morphotypic ranges. Thus tables present the ranges of morphotypes from which a list of events is constructed which leads to a range chart for Site 173 .

Although the interplay between evolution and ecology in determining vertical sequences of fossil organisms is generally well known, it is of particular significance for some of the sites cored on Leg 18. Sites 173 through 176 underlie the California current where mixing of various neighboring water masses take place. The southward flowing surface current brings a major component from the subarctic region while components from the Central Water Mass and, to a lesser extent, the Equatorial Water Mass are also present (Ried, Roden, and Wyllie, 1958). Furthermore, fluctuations in the relative importance of these various components take place on various scales from days to seasonal and longer terms. Thus one must be cautious in interpreting vertical sequences from limited numbers of locations. This is evident in the discontinuous ranges of 
many species and in apparent restricted ranges of species known to have longer ranges elsewhere.

\section{BIOSTRATIGRAPHIC FRAMEWORK}

Because of the mixing of equatorial and high-latitude assemblages, zonations proposed for both regions have been employed for parts of the Leg 18 sequences where they are applicable. In addition, the Miocene-Pliocene boundary for Site 173 is estimated on the basis of published and unpublished knowledge of radiolarian sequences in nearby land sections where other kinds of microfossils cooccur. This results in discontinuous coverage but is the best approximation of radiolarian biostratigraphy possible on the basis of existing knowledge. Although potential new biostratigraphic events are suggested by this study, too little information is available to permit description of new zones at this time. A brief discussion of the zones, stage-boundary levels, and radiometric-paleometric dates used for this report follows:

Miocene. The only well-preserved Miocene radiolarian sequence recovered during Leg 18 was at Site 173 (off Cape Mendocino, California). Here the following equatorial zones of Riedel and Sanfilippo $(1970,1971)$ could be recognized, but their boundaries could not be determined: Calocycletta costata, Dorcadospyris alata, Cannartus petterssoni, and Ommatartus antepenultimus.

Pliocene. The base of the Pliocene in the Pacific Coast region is tentatively taken as the earliest appearance of Lamprocyrtis heteroporos (= Lamprocyclas heteroporos). This level occurs near the top of the Capistrano formation at Newport Bay, California (unpublished data), at a level approximating the Miocene-Pliocene boundary based on foraminifera (Ingle, 1967; 1972). At Malaga Cove, California, it occurs in the Malaga Mudstone with the Delmontian stage according to Casey et al. (1972) and Casey (in press). The base of the upper Pliocene Lamprocyrtis heteroporos (Hays, 1970) Zone can be recognized at the top of the range of Stichocorys peregrina, as defined by Hays, with a radiometric-paleomagnetic age of 2.8 m.y. This leaves a segment of time from the Miocene-Pliocene boundary to the base of the $L$. heteroporos Zone representing part of the Spongaster pentas Zone of Riedel and Sanfilippo (1970) but not recognizable in this region.

Pleistocene. For the Gulf of Alaska sites (178-182) the Pleistocene zonation with estimated radiometric paleomagnetic ages of Hays (1970) were used. The base of the Eucyrtidium matuyamai Zone is taken as the base of the Olduvai paleomagnetic event with an age of $1.8 \mathrm{~m}$.y. and as the Pliocene-Pleistocene boundary. The age of $1.8 \mathrm{~m} . \mathrm{y}$. corresponds more closely to current usage for the base of the Olduvai and is now preferred by Hays (oral communication, 1972). The base of the Axoprunum angelinum (= Stylatractus universus) Zone is taken as the base of the Jaramillo event at 0.9 m.y.; the base of the Artostrobium miralestense (= Eucyrtidium tumidulum) Zone is taken as $0.4 \mathrm{~m} . \mathrm{y}$.; and the top of the Stylacontarium acquilonium (= Druppratractus acquilonius) Zone, which occurs consistently above the top of $A$. angelinum, is taken as 0.3 m.y. as estimated by Hays.

For the Pacific Coast sites (173-177), Hays' North Pacific Pleistocene zones are also used but with some modification. At Site 173, Lamprocyrtis heteroporos ranges above the first appearance of Eucyrtidium matuyamai, its extinction level in the North Pacific. Therefore the first evolutionary appearance of $E$. matuyamai is taken as the top of the $L$. heteroporos Zone and base of the overlying $E$. matuyami Zone. This level appears consistent with the first appearance of E. matuyamai in the North Pacific and agrees more closely with estimates of the Pliocene-Pleistocene boundary based on other microfossils at Site 173 than does the top of Lamprocyrtis heteroporos. Radiometricpaleomagnetic dates are assumed to be the same as in the Gulf of Alaska, though comparison with the paleomagnetic results from this site (Heinrichs, this volume) may reveal some disagreement due to differing species ranges.

\section{RADIOLARIAN SITE SUMMARIES}

Radiolarian results are summarized for each site with an accompanying table of the species encountered in each sample examined. The species listed are only those that could be identified with some confidence and constitute but a small proportion of the total number of species present. In the tables, the following abbreviations for abundance are applied: $R$, rare; $F$, few; $C$, common; $A$, abundant; plus sign, one or two specimens per slide; minus sign, no specimens in a sample after a concentrated search. Barren samples in the tabulated sequences are listed separately (Table 1) for the purpose of identifying short barren intervals in otherwise fossiliferous sequences. Major barren intervals are mentioned in the summaries without listing the samples examined, which include at least one sample per core (usually the core catcher).

TABLE 1

List of Barren Samples in Tabulated Sequences

\begin{tabular}{|c|c|}
\hline 175-19 (CC) & $178-29-3(41-43)$ \\
\hline $175-21$ (CC) & $178-29-4(34-36)$ \\
\hline $175-22(\mathrm{CC})$ & $178-29(\mathrm{CC})$ \\
\hline $176-5-1(73-75)$ & $\begin{array}{l}178-33-2(12-14) \\
178-33-3(47-49)\end{array}$ \\
\hline $177 \mathrm{~A}-10(\mathrm{CC})$ & $178-39-2(86-88)$ \\
\hline $178-23(\mathrm{CC})$ & $178-39-3(35-37)$ \\
\hline $178-24-1(102-104)$ & $178-39-4(37-39)$ \\
\hline $178-24-2(67-69)$ & $178-42(\mathrm{CC})$ \\
\hline $178-24-3(66-68)$ & $178-43-3(140-142)$ \\
\hline $178-24$ (CC) & $178-43$ (CC) \\
\hline $178-25-1(28-30)$ & $\begin{array}{l}178-45 \text { (CC) } \\
178-46-2(94-05)\end{array}$ \\
\hline $178-25-2(38-40)$ & $\begin{array}{l}178-46-2(94-95) \\
178-46 \text { (CC) }\end{array}$ \\
\hline $\begin{array}{l}178-25-3(88-90) \\
178-25-4(35-37)\end{array}$ & $178-47-1(128-130)$ \\
\hline $\begin{array}{l}178-25-4(35-37) \\
178-25-5(38-40)\end{array}$ & $178-47-2(79-82)$ \\
\hline $178-25$ (CC) & $178-47(\mathrm{CC})$ \\
\hline $178-26-2(54-56)$ & $178-48-1(72-74)$ \\
\hline $178-26-3(38-40)$ & 178-48 (CC) \\
\hline $178-26(\mathrm{CC})$ & $178-49-1(73-75)$ \\
\hline $178-27-1(100-102)$ & $\begin{array}{l}178-49 \text { (CC) } \\
178-508-100\end{array}$ \\
\hline $178-27$ (CC) & $\begin{array}{l}178-51-1(98-100) \\
178-51-2(69-71)\end{array}$ \\
\hline $178-28-1(64-66)$ & $\begin{array}{l}178-51-2(69-71) \\
178-51(\mathrm{CC})\end{array}$ \\
\hline $178-28-3(90-92)$ & $178-53(\mathrm{CC})$ \\
\hline $178-28-5(105-107)$ & $178-54(\mathrm{CC})$ \\
\hline & $179-5-6(63-65)$ \\
\hline
\end{tabular}


In the column headed Abundance, total radiolarian abundance is noted using the same abbreviations as above and based on sieved $(>62 \mu)$, acid-treated preparations. Overall radiolarian preservation is estimated as poor $(P)$, moderate $(M)$, or good $(G)$ in the column headed Preservation.

In the column headed $\mathrm{Age}$, series/age names are entered based on the best estimates of correlation of radiolarian zones and species ranges with these standards. A dashed line indicates a tentative boundary. The column headed Zone contains names of previously described radiolarian zones. Miocene zones can be recognized at Site 173, but their boundaries are not determinable.

While blank spaces in the tables usually suggest absence of species in samples, an artificial source of incompleteness in the tables should be pointed out. This stems from growth of the list of identifiable species as the study progressed and the lack of time to resurvey all samples for the added species. As nearly as possible, samples in critical areas (ends of ranges) were reexamined, but short gaps in species ranges must be interpreted with caution. Extended gaps, especially beyond apparent ends of ranges are more likely to be real.

Site $172\left(31^{\circ} 32^{\prime} \mathrm{N}, 133^{\circ} 22^{\prime} \mathrm{W}\right.$; water depth $\left.4768 \mathrm{~m}\right)$. Radiolarians occur only near the top of Hole 172 . The core catcher of Core 1 contains rare, well-preserved specimens. The following species could be recognized: Theocorythium sp. cf. T. trachelium (Ehrenberg), Siphonosphaera polysiphonia (Haeckel), Heliodiscus asteriscus (Haeckel), Eucyrtidium acuminatum (Ehrenberg), Artostrobium miralestense (Campbell and Clark), and Euchitonia elegans (Ehrenberg). As samples above the core catcher contain no radiolarians, these may represent contamination from the surface sediment. These are all living species and, in the absence of diagnostic older species, the assemblage is considered Quaternary. Very rare fragments in Core 3 are barely recognizable as radiolarians. Other portions of Holes 172 and $172 \mathrm{~A}$ are barren.

Site $173\left(39^{\circ} 58^{\prime} \mathrm{N}, 125^{\circ} 27^{\prime} \mathrm{W}\right.$; water depth $\left.1927 \mathrm{~m}\right)$ (Table 2). Radiolarians are abundant and well-preserved throughout most of Hole 173 from the sea floor to about

TABLE 2A

Radiolarians at Site 173

\begin{tabular}{|c|c|c|c|c|c|c|c|c|c|c|c|c|c|c|c|c|c|c|}
\hline Sample & 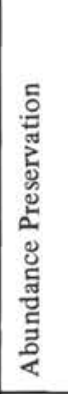 & 喿 & Бّ & 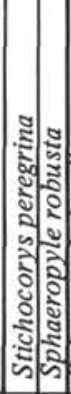 & 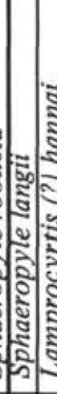 & 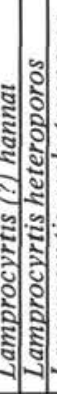 & 告 & : & : & 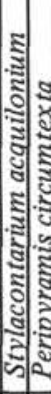 & 영 & 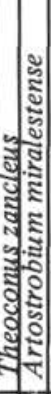 & 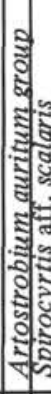 & & 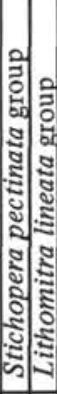 & 新 & : & 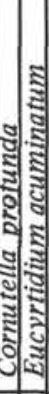 \\
\hline $\begin{array}{l}173-1-2(102-104) \\
173-1(C C) \\
173-2-2(45-47) \\
173-2-2(118-120) \\
173-2-3(40-42)\end{array}$ & $\begin{array}{l}\mathrm{A}, \mathrm{G} \\
\mathrm{C}, \mathrm{G} \\
\mathrm{A}, \mathrm{G} \\
\mathrm{C}, \mathrm{G} \\
\mathrm{C}, \mathrm{P}\end{array}$ & \multirow{5}{*}{ 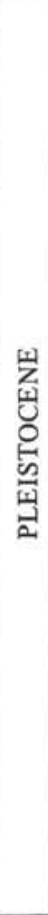 } & 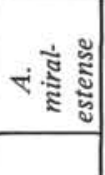 & & $\mathrm{F}$ & $\mathrm{R}$ & & + & $\begin{array}{l}- \\
- \\
- \\
- \\
F\end{array}$ & $\begin{array}{ll}- & F \\
- & F \\
- & R \\
- & R \\
+ & R\end{array}$ & $\mid \begin{array}{ll}\mathrm{R} \\
\mathrm{R}\end{array}$ & 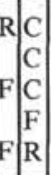 & $\mathrm{F} F$ & & $\begin{array}{l}\mathrm{R} \\
\mathrm{R} \\
\mathrm{R}\end{array}$ & \begin{tabular}{|l|l|}
$C$ & $F$ \\
$C$ & $F$ \\
$F$ & $R$ \\
& \\
$R$ & $R$
\end{tabular} & $\mid \begin{array}{lll}\mathrm{F} & + \\
\mathrm{F} & \mathrm{F} \\
\mathrm{F} & \mathrm{R} \\
\mathrm{F} & \mathrm{R} \\
\mathrm{F} & & \\
\end{array}$ & $\begin{array}{l}+ \\
F \\
\text { R } \\
\text { R }\end{array}$ \\
\hline $\begin{array}{l}173-2-4(45-47) \\
173-2-5(65-67) \\
173-2-6(56-58) \\
173-2(\mathrm{CC}) \\
173-3-1(45-47)\end{array}$ & $\begin{array}{l}\mathrm{G} \\
\mathrm{M} \\
\mathrm{M} \\
\mathrm{G} \\
\mathrm{G}\end{array}$ & & \multirow{3}{*}{ 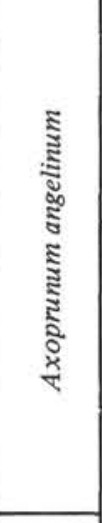 } & & $\mathrm{R}$ & & \begin{tabular}{|l|l} 
& $F$ \\
$R$ \\
$R$ \\
$R$ \\
$F$
\end{tabular} & $\begin{array}{l}R \\
R\end{array}$ & $\begin{array}{l}\mathrm{F} \\
\mathrm{R} \\
\mathrm{R} \\
\mathrm{R}\end{array}$ & \begin{tabular}{|l|l} 
& $\mathrm{F}$ \\
$\mathrm{R}$ & \\
$\mathrm{R}$ & \\
$\mathrm{R}$ & \\
$\mathrm{R}$ & $\mathrm{F}$
\end{tabular} & $\mathrm{R}$ & \begin{tabular}{l|l}
$C$ & $F$ \\
$R$
\end{tabular} & \begin{tabular}{l|l}
$R$ & $F$
\end{tabular} & & $\mathrm{R}$ & $\mathrm{C} F$ & $F$ & $\mathrm{R} F$ \\
\hline $\begin{array}{l}173-3-3(106-108) \\
173-3(\mathrm{CC}) \\
173-4-1(57-59) \\
173-4-2(57-59) \\
173-4-3(57-59)\end{array}$ & $\begin{array}{l}\text { C,G } \\
\text { F,G } \\
\text { A,G } \\
\text { A,G } \\
\text { A,G }\end{array}$ & & & & 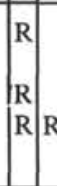 & & \begin{tabular}{l|l}
$\mathrm{F}$ & $\mathrm{C}$ \\
$\mathrm{R}$ & $\mathrm{F}$ \\
$\mathrm{R}$ & $\mathrm{F}$ \\
$\mathrm{F}$ & $\mathrm{F}$ \\
$\mathrm{C}$ & $\mathrm{R}$
\end{tabular} & $\begin{array}{ll}R \\
R \\
R \\
R\end{array}$ & $\begin{array}{l}\mathrm{F} \\
\mathrm{R} \\
\mathrm{R} \\
\mathrm{R} \\
\mathrm{R}\end{array}$ & \begin{tabular}{|l|l}
$\mathrm{R}$ & $\mathrm{F}$ \\
$\mathrm{R}$ & $\mathrm{R}$ \\
$\mathrm{R}$ & $\mathrm{R}$ \\
$\mathrm{R}$ & $\mathrm{R}$ \\
$\mathrm{F}$ & $\mathrm{F}$ \\
\end{tabular} & $\begin{array}{lll}+ & F \\
F & F \\
R & F \\
R & F \\
R & F\end{array}$ & $\mathrm{~F} F \mid \begin{array}{l}\mathrm{R} \\
\mathrm{C} \\
\mathrm{F} \\
\mathrm{C} \\
\mathrm{C}\end{array}$ & \begin{tabular}{|l|l}
$\mathrm{R}$ & $\mathrm{R}$ \\
$\mathrm{F}$ & $\mathrm{R}$ \\
$\mathrm{R}$ & $\mathrm{F}$ \\
$\mathrm{R}$
\end{tabular} & R. & $\begin{array}{l}+ \\
\mathrm{R} \\
\mathrm{R}\end{array}$ & \begin{tabular}{l|l}
$\mathrm{C}$ & $\mathrm{F}$ \\
$\mathrm{R}$ & $\mathrm{F}$ \\
$\mathrm{C}$ & $\mathrm{C}$ \\
$\mathrm{C}$ & $\mathrm{R}$ \\
$\mathrm{C}$ & $\mathrm{F}$ \\
\end{tabular} & \begin{tabular}{|lll}
$R$ & \\
$C$ & $R$ \\
$R$ & \\
$R$ & \\
$F$ & $R$ \\
\end{tabular} & \begin{tabular}{l|l}
$R$ & $F$ \\
$R$ \\
$R$ \\
$R$ \\
$R$
\end{tabular} \\
\hline $\begin{array}{l}173-4-4(57-59) \\
173-4-5(57-59) \\
173-4(C C) \\
173-5-1(120-122) \\
173-5-2(57-59)\end{array}$ & $\begin{array}{l}\mathrm{A}, \mathrm{G} \\
\mathrm{A}, \mathrm{G} \\
\mathrm{C}, \mathrm{G} \\
\mathrm{A}, \mathrm{G} \\
\mathrm{C}, \mathrm{G}\end{array}$ & & & & \begin{tabular}{l|l}
$\mathrm{R}$ & $\mathrm{R}$ \\
$\mathrm{R}$ & $\mathrm{R}$ \\
$\mathrm{F}$ & $\mathrm{R}$ \\
$\mathrm{R}$ &
\end{tabular} & $\mathrm{R} /+\left(\begin{array}{ll}\mathrm{R} \\
\mathrm{R} \\
\mathrm{R} \\
+ \\
1 \\
1 \\
1 \\
1\end{array}\right.$ & \begin{tabular}{|l|l}
$\mathrm{F}$ & $\mathrm{R}$ \\
$\mathrm{F}$ & $\mathrm{R}$ \\
$\mathrm{F}$ & $\mathrm{R}$ \\
$\mathrm{F}$ & $\mathrm{R}$ \\
$\mathrm{R}$ & +
\end{tabular} & - & $+\begin{array}{l}\mathrm{R} \\
\mathrm{R} \\
\mathrm{F} \\
\mathrm{F} \\
\mathrm{F}\end{array}$ & \begin{tabular}{l|l}
$R$ & $R$ \\
$F$ & $R$ \\
$C$ & $F$ \\
$F$ & $R$ \\
$F$ & $R$
\end{tabular} & $\begin{array}{ll}R & C \\
R & \\
F \\
R \\
R\end{array}$ & $\mathrm{C}=\begin{array}{l}\mathrm{C} \\
\mathrm{F} \\
\mathrm{C} \\
\mathrm{C} \\
\mathrm{F}\end{array}$ & \begin{tabular}{|l|l} 
& $\mathrm{R}$ \\
$\mathrm{R}$ & $\mathrm{R}$ \\
$\mathrm{R}$ & $\mathrm{C}$ \\
$\mathrm{R}$ & $\mathrm{R}$ \\
&
\end{tabular} & & \begin{tabular}{l|l}
$\mathrm{R}$ & \\
$\mathrm{R}$ & \\
$\mathrm{R}$ & $\mathrm{R}$
\end{tabular} & \begin{tabular}{|l|l|}
$\mathrm{C}$ & $\mathrm{R}$ \\
$\mathrm{C}$ & $\mathrm{R}$ \\
$\mathrm{F}$ & $\mathrm{R}$ \\
$\mathrm{C}$ & $\mathrm{F}$ \\
$\mathrm{C}$ & $\mathrm{F}$
\end{tabular} & \begin{tabular}{|l|l}
$R$ & \\
$F$ & \\
$C$ & $R$ \\
$F$ & $R$
\end{tabular} & \begin{tabular}{l|l}
$R$ & $R$ \\
$R$ & $C$
\end{tabular} \\
\hline $\begin{array}{l}173-5-3(57-59) \\
173-5-4(57-59) \\
173-5-5(57-59) \\
173-5(\mathrm{CC}) \\
173-6-1(108-110)\end{array}$ & $\begin{array}{l}\mathrm{C}, \mathrm{G} \\
\mathrm{A}, \mathrm{G} \\
\mathrm{A}, \mathrm{G} \\
\mathrm{C}, \mathrm{G} \\
\mathrm{C}, \mathrm{M}\end{array}$ & & 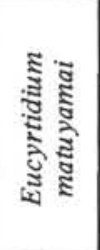 & & \begin{tabular}{|l|l}
$\mathrm{F}$ & $\mathrm{R}$ \\
$\mathrm{F}$ & $\mathrm{R}$ \\
$\mathrm{R}$ & $\mathrm{R}$ \\
$\mathrm{R}$
\end{tabular} & \begin{tabular}{l|ll}
$R$ & 1 \\
$R$ & $R$ & 1 \\
$R$ & $R$ & 1 \\
$R$ & 1 \\
+ & 1
\end{tabular} & $\mid \begin{array}{l}F \\
F \\
F \\
F \\
F\end{array}$ & $+\begin{array}{l}r \\
C \\
+\end{array}$ & $+\begin{array}{l}\mathrm{F} \\
\mathrm{F} \\
\mathrm{F} \\
\mathrm{R} \\
+\mathrm{F} \\
\mathrm{F} \\
\mathrm{R}\end{array}$ & \begin{tabular}{l|l}
$\mathrm{C}$ & $\mathrm{F}$ \\
$\mathrm{F}$ & $\mathrm{F}$ \\
$\mathrm{F}$ & $\mathrm{R}$ \\
$\mathrm{F}$ & $\mathrm{F}$ \\
$\mathrm{F}$ & $\mathrm{R}$
\end{tabular} & \begin{tabular}{|lll}
$R$ & $F$ \\
$R$ & $F$ \\
$R$ & $C$ \\
$F$ & \\
$F$ & $F$ \\
$R$
\end{tabular} & \begin{tabular}{l|l}
$\mathrm{F}$ \\
$\mathrm{F}$ \\
$\mathrm{C}$ \\
$\mathrm{C}$ \\
$\mathrm{C}$ \\
$\mathrm{C}$ \\
$\mathrm{F}$
\end{tabular} & \begin{tabular}{|l|l} 
& $R$ \\
$F$ & $F$ \\
$C$ & \\
$F$ & $F$
\end{tabular} & & $\mathrm{R}$ & \begin{tabular}{l|l}
$C$ & $R$ \\
$C$ & $R$ \\
$C$ & $R$ \\
$C$ & $F$ \\
$C$ & $F$ \\
\end{tabular} & $\mid$\begin{tabular}{l|l}
$\mathrm{F}$ & $\mathrm{R}$ \\
$\mathrm{R}$ & \\
$\mathrm{R}$ & \\
$\mathrm{C}$ & $\mathrm{R}$ \\
$\mathrm{F}$ & $\mathrm{R}$ \\
\end{tabular} & \begin{tabular}{l|l}
$\mathrm{R}$ & $\begin{array}{l}\mathrm{R} \\
\mathrm{R} \\
\mathrm{R}\end{array}$ \\
$\mathrm{R}$
\end{tabular} \\
\hline
\end{tabular}


TABLE 2A - Continued

\begin{tabular}{|c|c|c|c|c|c|c|c|c|c|c|c|c|c|c|c|c|c|c|c|}
\hline $\begin{array}{l}173-6-2(55-57) \\
173-6-3(57-59) \\
173-6 \text { (CC) } \\
173-7-1(69-71) \\
173-7-2(57-59)\end{array}$ & $\begin{array}{l}\mathrm{A}, \mathrm{M} \\
\mathrm{A}, \mathrm{M} \\
\mathrm{C}, \mathrm{G} \\
\mathrm{A}, \mathrm{M}\end{array}$ & \multirow{3}{*}{ 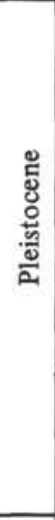 } & \multirow{3}{*}{ 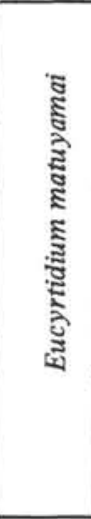 } & & \begin{tabular}{|l|ll} 
& & 1 \\
$R$ & 1 \\
1 & 1 \\
$R$ & 1 & \\
$R$ & 1
\end{tabular} & \begin{tabular}{l|l|l}
$\mathrm{R}$ & $\mathrm{F}$ & $\mathrm{R}$ \\
$\mathrm{R}$ & $\mathrm{F}$ & $\mathrm{R}$ \\
$\mathrm{R}$ & $\mathrm{F}$ & $\mathrm{R}$ \\
$\mathrm{R}$ & $\mathrm{F}$ & $\mathrm{R}$ \\
$\mathrm{R}$ & $\mathrm{F}$ & $\mathrm{R}$ \\
$\mathrm{R}$ & $\mathrm{R}$
\end{tabular} & $\mathrm{R}$ & \begin{tabular}{l|l}
$\mathrm{F}$ & $\mathrm{F}$ \\
$\mathrm{F}$ & $\mathrm{F}$ \\
$\mathrm{C}$ & $\mathrm{R}$ \\
$\mathrm{F}$ & $\mathrm{R}$ \\
$\mathrm{F}$ & $\mathrm{R}$
\end{tabular} & $\mid \begin{array}{l}\mathrm{F} \\
\mathrm{F} \\
\mathrm{R} \\
\mathrm{R} \\
\mathrm{R} \\
\mathrm{R}\end{array}$ & \begin{tabular}{l|l}
$\mathrm{F}$ & $\mathrm{F}$ \\
$\mathrm{R}$ & $\mathrm{F}$ \\
$\mathrm{F}$ & $\mathrm{F}$ \\
$\mathrm{R}$ & $\mathrm{F}$ \\
$\mathrm{R}$ & $\mathrm{F}$
\end{tabular} & \begin{tabular}{l|l}
$R$ & $F$ \\
$R$ & \\
$R$ & \\
$R$ & $R$ \\
$R$ & $R$
\end{tabular} & \begin{tabular}{l|l}
$\mathrm{F}$ & $\mathrm{F}$ \\
$\mathrm{F}$ & \\
$\mathrm{C}$ & $\mathrm{F}$ \\
$\mathrm{F}$ & $\mathrm{F}$ \\
& $\mathrm{F}$
\end{tabular} & \begin{tabular}{l|l}
$\mathrm{F}$ & $\mathrm{C}$ \\
$\mathrm{R}$ & $\mathrm{C}$ \\
$\mathrm{F}$
\end{tabular} & & $\mathrm{R}$ & \begin{tabular}{l|l}
$\mathrm{A}$ & \\
$\mathrm{C}$ & \\
$\mathrm{C}$ & $\mathbf{C}$ \\
$\mathrm{C}$ & $\mathrm{C}$ \\
$\mathrm{C}$ &
\end{tabular} & \begin{tabular}{l|l}
$\mathrm{R}$ & $\mathrm{F}$ \\
$\mathrm{R}$ & $\mathrm{F}$ \\
$\mathrm{F}$ & $\mathrm{C}$ \\
$\mathrm{R}$ & \\
$\mathrm{R}$ & $\mathrm{F}$
\end{tabular} & R & $\mathrm{R}$ \\
\hline $\begin{array}{l}173-7-3(57-59) \\
173-7-4(57-59) \\
173-8-1(62-64) \\
173-8-2(57-59) \\
173-8-3(57-59)\end{array}$ & $\begin{array}{l}\mathrm{M} \\
\mathrm{G} \\
\mathrm{G} \\
\mathrm{M} \\
\mathrm{M}\end{array}$ & & & & $\begin{array}{ll}\mathrm{R} \\
\mathrm{R}\end{array}$ & 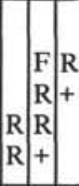 & + & \begin{tabular}{l|l}
$\mathrm{F}$ & $\mathrm{F}$ \\
$\mathrm{R}$ & $\mathrm{R}$ \\
$\mathrm{R}$ & $\mathrm{F}$ \\
$\mathrm{F}$ & $\mathrm{F}$ \\
$\mathrm{F}$ &
\end{tabular} & $\begin{array}{l}F \\
R \\
R \\
R \\
F\end{array}$ & \begin{tabular}{|l|l}
$\mathrm{F}$ & $\mathrm{F}$ \\
$\mathrm{R}$ & $\mathrm{F}$ \\
$\mathrm{R}$ & $\mathrm{F}$ \\
$\mathrm{R}$ & $\mathrm{F}$ \\
$\mathrm{R}$ & $\mathrm{F}$ \\
\end{tabular} & \begin{tabular}{l|l}
$R$ & \\
$R$ & $F$ \\
$R$ & $F$ \\
$R$ & $F$ \\
$R$ &
\end{tabular} & \begin{tabular}{|l|l}
$\mathrm{F}$ & \\
$\mathrm{C}$ & \\
$\mathrm{C}$ & $\mathrm{F}$ \\
$\mathrm{F}$ & \\
$\mathrm{F}$ & $\mathrm{F}$
\end{tabular} & \begin{tabular}{l|l}
$R$ & $R$ \\
$R$ \\
$R$
\end{tabular} & & + & $\begin{array}{l}A \\
C \\
C \\
C \\
C\end{array}$ & \begin{tabular}{l|l}
$\mathrm{F}$ & \\
$\mathrm{R}$ & $\mathrm{F}$ \\
+ & $\mathrm{F}$ \\
$\mathrm{R}$ & $\mathrm{F}$ \\
$\mathrm{F}$ & $\mathrm{F}$
\end{tabular} & $\begin{array}{l}R \\
R \\
R\end{array}$ & $\begin{array}{l}R \\
R \\
R \\
R\end{array}$ \\
\hline $\begin{array}{l}173-8-4(57-59) \\
173-8-5(57-59) \\
173-8-6(57-59) \\
173-9-1(57-59) \\
173-9-2(57-59)\end{array}$ & & & & & $\begin{array}{lll}R & \\
R & & \\
R & & \end{array}$ & $\mathrm{~F}\left|\begin{array}{l}\mathrm{R} \\
\mathrm{F} \\
\mathrm{F} \\
\mathrm{F} \\
\mathrm{F} \\
\mathrm{F}\end{array}\right|+$ & $\mathrm{F}$ & \begin{tabular}{l|l}
$R$ & $R$ \\
$F$ & $F$ \\
$F$ & $R$ \\
$F$ & $R$ \\
& $R$
\end{tabular} & \begin{tabular}{|l|l}
$R$ \\
$F$ \\
$R$ \\
$R$ \\
$R$ \\
$R$
\end{tabular} & \begin{tabular}{|l|l}
$\mathrm{R}$ & $\mathrm{F}$ \\
$\mathrm{R}$ & $\mathrm{F}$ \\
$\mathrm{R}$ & $\mathrm{F}$ \\
$\mathrm{R}$ & $\mathrm{F}$ \\
$\mathrm{R}$ & $\mathrm{F}$
\end{tabular} & \begin{tabular}{l|l}
$R$ & $R$ \\
$R$ & $R$ \\
$R$ \\
$R$ \\
$R$
\end{tabular} & \begin{tabular}{l|l}
$\mathrm{C}$ & $\mathrm{F}$ \\
$\mathrm{F}$ & $\mathrm{F}$ \\
$\mathrm{F}$ & $\mathrm{F}$ \\
$\mathrm{F}$ & $\mathrm{F}$ \\
$\mathrm{F}$ & $\mathrm{F}$
\end{tabular} & \begin{tabular}{l|l}
$F$ & $R$ \\
$R$ & $R$ \\
$R$ & $R$ \\
$R$ & $R$ \\
$F$ & +
\end{tabular} & & + & $\begin{array}{l}c \\
c \\
c \\
c \\
c\end{array}$ & \begin{tabular}{l|l} 
& $\mathrm{F}$ \\
$\mathrm{R}$ & \\
$\mathrm{R}$ & \\
$\mathrm{R}$ & $\mathrm{F}$ \\
$\mathrm{R}$ & $\mathrm{F}$
\end{tabular} & + & $R$ \\
\hline & & \multirow{6}{*}{$\begin{array}{l}\text { Еँّ } \\
\stackrel{\Xi}{0}\end{array}$} & \multirow{4}{*}{ 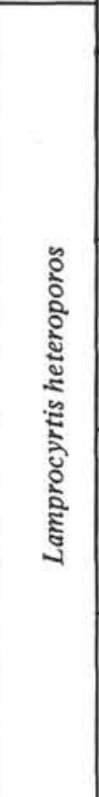 } & $\begin{array}{l}\mathrm{R} \\
\mathrm{R}\end{array}$ & $\begin{array}{l}R \\
\mathrm{R} \\
\mathrm{R}\end{array}$ & $\begin{array}{l}\mathrm{R} \\
\mathrm{F} \\
\mathrm{F} \\
\mathrm{C} \\
\mathrm{C}\end{array}$ & $\begin{array}{l}\mathrm{F} \\
\mathrm{F} \\
\mathrm{R} \\
\mathrm{F} \\
\mathrm{F}\end{array}$ & \begin{tabular}{l|l}
$R$ \\
$R$ \\
$R$ \\
$R$ \\
$R$
\end{tabular} & \begin{tabular}{l|l}
$R$ & $R$ \\
$R$ \\
$R$ \\
$R$ \\
$R$ \\
$R$ \\
\\
$F$
\end{tabular} & \begin{tabular}{l|l}
$\mathrm{R}$ & $\mathrm{F}$ \\
$\mathrm{R}$ & $\mathrm{F}$ \\
$\mathrm{R}$ & $\mathrm{F}$ \\
$\mathrm{R}$ & $\mathrm{F}$ \\
$\mathrm{R}$ & $\mathrm{F}$
\end{tabular} & \begin{tabular}{l|l}
$R$ & \\
$R$ & \\
$R$ & $R$ \\
$R$ & $R$ \\
$R$ &
\end{tabular} & 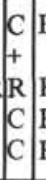 & \begin{tabular}{l|l}
$\mathrm{F}$ & $\mathrm{F}$ \\
$\mathrm{R}$ & + \\
$\mathrm{R}$ & $\mathrm{R}$ \\
$\mathrm{R}$ & $\mathrm{R}$
\end{tabular} & & $\mathrm{R}{ }_{+}$ & $\begin{array}{l}\mathrm{C} \\
\mathrm{C} \\
\mathrm{C} \\
\mathrm{C} \\
\mathrm{F}\end{array}$ & \begin{tabular}{l|l}
$\mathrm{F}$ & $\mathrm{F}$ \\
$\mathrm{R}$ & $\mathrm{F}$ \\
$\mathrm{R}$ & $\mathrm{F}$ \\
+ & $\mathrm{F}$ \\
$\mathrm{R}$ & $\mathrm{F}$
\end{tabular} & $\begin{array}{l}R \\
+ \\
R\end{array}$ & $\begin{array}{l}R \\
R \\
R \\
R \\
R\end{array}$ \\
\hline $\begin{array}{l}173-10-2(57-59) \\
173-10-3(57-59) \\
173-10-4(57-59) \\
173-10-5(57-59) \\
173-10-6(57-59)\end{array}$ & & & & & & $\begin{array}{l}\mathrm{C} \\
\mathrm{C} \\
\mathrm{C} \\
\mathrm{C} \\
\mathrm{F}\end{array}$ & $\begin{array}{l}\mathrm{F} \\
\mathrm{F} \\
\mathrm{F} \\
\mathrm{F} \\
+\end{array}$ & $\begin{array}{l}\mathrm{R} \\
\mathrm{R} \\
\mathrm{R} \\
\mathrm{R}\end{array}$ & \begin{tabular}{l|l|}
$R$ & $F$ \\
$R$ \\
$R$ \\
$R$ \\
$R$ \\
$R$ \\
$R$
\end{tabular} & \begin{tabular}{l|l}
$\mathrm{R}$ & $\mathrm{F}$ \\
$\mathrm{R}$ & $\mathrm{F}$ \\
$\mathrm{R}$ & $\mathrm{F}$ \\
$\mathrm{R}$ & $\mathrm{F}$ \\
$\mathrm{R}$ & $\mathrm{F}$
\end{tabular} & $\begin{array}{l}\mathrm{R} \\
\mathrm{R} \\
\mathrm{R} \\
\mathrm{R} \\
\mathrm{R} \\
\mathrm{R}\end{array}$ & \begin{tabular}{|l|l}
$\mathrm{C}$ & \\
$\mathrm{F}$ & $\mathrm{F}$ \\
$\mathrm{F}$ & \\
$\mathrm{F}$ & \\
$\mathrm{F}$ &
\end{tabular} & \begin{tabular}{l|l}
$R$ & $R$ \\
$F$ & $F$ \\
$R$ \\
$R$ \\
$R$
\end{tabular} & & $\begin{array}{l}+ \\
\mathrm{R}\end{array}$ & \begin{tabular}{|l|}
$\mathrm{F}$ \\
$\mathrm{C}$ \\
$\mathrm{F}$ \\
$\mathrm{R}$ \\
$\mathrm{C}$
\end{tabular} & \begin{tabular}{|l|l}
$\mathrm{R}$ & $\mathrm{F}$ \\
$\mathrm{F}$ & $\mathrm{F}$ \\
& $\mathrm{F}$ \\
$\mathrm{R}$ & $\mathrm{F}$ \\
$\mathrm{R}$ & $\mathrm{F}$
\end{tabular} & & $\begin{array}{l}R \\
F \\
R\end{array}$ \\
\hline $\begin{array}{l}173-11-1(58-60) \\
173-11-2(57-59) \\
173-11-3(57-59) \\
173-11-4(57-59) \\
173-12-1(57-59)\end{array}$ & $\begin{array}{l}\mathrm{A}, \mathrm{M} \\
\mathrm{A}, \mathrm{M} \\
\mathrm{A}, \mathrm{M} \\
\mathrm{C}, \mathrm{M} \\
\mathrm{A}, \mathrm{M}\end{array}$ & & & & & $\begin{array}{l}\mathrm{C} \\
\mathrm{C} \\
\mathrm{C} \\
\mathrm{F} \\
\mathrm{C}\end{array}$ & $\begin{array}{l}\mathrm{R} \\
\mathrm{F} \\
\mathrm{F} \\
\mathrm{F} \\
\mathrm{R}\end{array}$ & $\begin{array}{l}F \\
F \\
F \\
R\end{array}$ & $\begin{array}{l}\mathrm{R} \\
\mathrm{R} \\
\mathrm{F} \\
\mathrm{R}\end{array}$ & \begin{tabular}{l|l}
$\mathrm{R}$ & $\mathrm{H}$ \\
$\mathrm{R}$ & $\mathrm{H}$ \\
$\mathrm{F}$ & $\mathrm{H}$ \\
$\mathrm{R}$ & \\
$\mathrm{R}$ & $\mathrm{H}$
\end{tabular} & $\begin{array}{l}\mathrm{R} \\
\mathrm{R} \\
\mathrm{R}\end{array}$ & \begin{tabular}{|l|l}
$\mathrm{F}$ & $\mathrm{r}$ \\
$\mathrm{F}$ & \\
$\mathrm{C}$ & $\mathrm{I}$ \\
$\mathrm{F}$ & $\mathrm{F}$
\end{tabular} & \begin{tabular}{l|l}
$R$ & \\
$F$ & $R$ \\
$R$ & $R$ \\
$R$ \\
$R$
\end{tabular} & + & $\mathrm{R}$ & $\begin{array}{l}\mathrm{C} \\
\mathrm{C} \\
\mathrm{F} \\
\mathrm{C} \\
\mathrm{C}\end{array}$ & \begin{tabular}{l|l}
$\mathrm{R}$ & \\
$\mathrm{R}$ & $\mathrm{F}$ \\
$\mathrm{R}$ & $\mathrm{F}$ \\
$\mathrm{R}$ & \\
& $\mathrm{F}$
\end{tabular} & & $\begin{array}{l}R \\
F \\
R \\
R\end{array}$ \\
\hline $\begin{array}{l}173-12-2(57-59) \\
173-12-3(57-59) \\
173-12-4(57-59) \\
173-12-5(65-67) \\
173-12-6(57-59)\end{array}$ & & & & & & $\begin{array}{l}\mathrm{C} \\
\mathrm{A} \\
\mathrm{C} \\
\mathrm{C} \\
\mathrm{C}\end{array}$ & $\begin{array}{l}\mathrm{R} \\
\mathrm{R} \\
\mathrm{R} \\
\mathrm{R} \\
\mathrm{F}\end{array}$ & 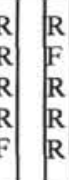 & $\begin{array}{lll}R \\
R \\
+ \\
R \\
+\end{array}$ & \begin{tabular}{l|l}
$\mathrm{R}$ & \\
$\mathrm{R}$ & $\mathrm{F}$ \\
$\mathrm{R}$ & $\mathrm{H}$ \\
$\mathrm{R}$ & $\mathrm{F}$ \\
$\mathrm{R}$ & $\mathrm{H}$
\end{tabular} & $\begin{array}{l}\mathrm{F} \\
\mathrm{R} \\
\mathrm{R} \\
\mathrm{R}\end{array}$ & \begin{tabular}{l|l}
$R$ & 1 \\
$F$ & 1 \\
$C$ & 1 \\
$F$ & 1 \\
$C$ & 1
\end{tabular} & \begin{tabular}{l|l}
$R$ & \\
$F$ & $R$ \\
$R$ & $R$ \\
$R$ & \\
$R$ & $R$
\end{tabular} & $+\begin{array}{l}+ \\
1 \\
1 \\
1 \\
+\end{array}$ & $\begin{array}{l}+ \\
\mathrm{R} \\
\mathrm{R}\end{array}$ & $\begin{array}{l}\mathrm{C} \\
\mathrm{F} \\
\mathrm{F} \\
\mathrm{F} \\
\mathrm{C}\end{array}$ & \begin{tabular}{l|l}
$R$ & $F$ \\
$R$ & \\
& $F$ \\
$R$ & $F$ \\
$R$ & $F$
\end{tabular} & & $\begin{array}{l}R \\
F \\
R \\
R \\
R\end{array}$ \\
\hline $\begin{array}{l}173-13-3(57-59) \\
173-14-1(57-59) \\
173-14-2(92-94) \\
173-14-3(57-59) \\
173-14-(\text { CC) }\end{array}$ & $\begin{array}{l}\mathrm{G} \\
\mathrm{G} \\
\mathrm{G} \\
\mathrm{M}\end{array}$ & & 递它 & \begin{tabular}{|l|l}
+ & \\
$\mathrm{R}$ & \\
$\mathrm{R}$ & $\mathrm{R}$
\end{tabular} & & \begin{tabular}{|l|l|}
$\mathrm{F}$ & $\mathrm{C}$ \\
$\mathrm{R}$ & $\mathrm{C}$ \\
$\mathrm{R}$ & $\mathrm{C}$ \\
$\mathrm{R}$ & $\mathrm{C}$ \\
$\mathrm{F}$ & \\
\end{tabular} & $\begin{array}{l}\mathrm{R} \\
\mathrm{R} \\
\mathrm{F} \\
\mathrm{R} \\
\mathrm{N}\end{array}$ & \begin{tabular}{l|l}
$\mathrm{R}$ & $\mathrm{R}$ \\
$\mathrm{R}$ & $\mathrm{R}$ \\
$\mathrm{F}$ & $\mathrm{R}$ \\
$\mathrm{R}$ \\
$\mathrm{R}$ \\
$\mathrm{R}$ \\
$\mathrm{R}$
\end{tabular} & \begin{tabular}{|l|l|}
$R$ & $R$ \\
$R$ & $R$ \\
$R$ & $R$ \\
$R$ & $R$ \\
$R$ & $R$ \\
\end{tabular} & \begin{tabular}{|l|l}
$\mathrm{F}$ & $\mathrm{F}$ \\
$\mathrm{R}$ & $\mathrm{H}$ \\
$\mathrm{R}$ & $\mathrm{H}$ \\
$\mathrm{R}$ & $\mathrm{F}$ \\
$\mathrm{R}$ & $\mathrm{F}$
\end{tabular} & $\begin{array}{l}\mathrm{R} \\
\mathrm{R} \\
\mathrm{R} \\
\mathrm{R} \\
\mathrm{R}\end{array}$ & \begin{tabular}{|l|l}
$\mathrm{F}$ & \\
$\mathrm{F}$ \\
$\mathrm{R}$ \\
$\mathrm{R}$ \\
$\mathrm{R}$ \\
$\mathrm{R}$
\end{tabular} & $\begin{array}{l}\mathrm{F} \\
\mathrm{R} \\
\mathrm{F} \\
\mathrm{R} \\
\mathrm{F}\end{array}$ & $\mathrm{F}$ & $\mathrm{R}$ & \begin{tabular}{|l}
$\mathrm{F}$ \\
$\mathrm{C}$ \\
$\mathrm{F}$
\end{tabular} & \begin{tabular}{l|l}
$\mathrm{F}$ & $\mathrm{H}$ \\
$\mathrm{R}$ & + \\
$\mathrm{F}$ & $\mathrm{H}$ \\
$\mathrm{R}$ & $\mathrm{H}$
\end{tabular} & & $\begin{array}{l}R \\
F \\
R \\
R \\
R\end{array}$ \\
\hline $173-15-2(57-59)$ & $\mathrm{A}, \mathrm{M}$ & & & \begin{tabular}{l|l}
$R$ & $R$ \\
\end{tabular} & & C & $\mathrm{R}$ & $\mathrm{R}$ & $R$ & & & & & & & & & & \\
\hline
\end{tabular}

285 meters. This represents the best section of Neogene radiolarians yet known from this region. The sequence is apparently uninterrupted from early Miocene to the present. Figure 1 shows the ranges of selected species at this site.

Cores 1 through 31 are rich in radiolarians of Neogene age. Cores 32,34 , and 35 contain poorly preserved (recrystallized) specimens, including some probably Cretaceous forms - "Dictyomitra multicostata" group, pseudoaulophacids - with minor quantities of wellpreserved Tertiary species. The recrystallized material was encountered only as free specimens, so it is not clear whether they are reworked into Tertiary sediment or represent the presence of an older formation near the bottom of the hole. The very small recovery of sediment in
Core 36 was not examined for radiolarians, and Core 37 is barren.

Orosphaerids are common through most of this core, but none were identified, and special preparations for their study were not made.

Site $174\left(44^{\circ} 53^{\prime} \mathrm{N}, 126^{\circ} 21^{\prime} \mathrm{W}\right.$; water depths 2815 and $2799 \mathrm{~m}$ ). A few well-preserved radiolarians occur sporadically in Holes 174 and 174A. Both abundance and diversity are low. No species diagnostic of pre-Quaternary ages was observed. A typical assemblage (from 174-1, CC) is Dictyophimus crisiae, Bathropyramis woodringi, Artostrobium miralstense, Theoconus zancleus, Lamprocyrtis haysi, and Sphaeropyle langii. The presence of Stylacontarium acquilonium (one specimen in 174-1, CC; rare in 174A-33, CC suggests a possible minimum age of 0.4 m.y., the top of 
TABLE 2B

Radiolarians at Site 173

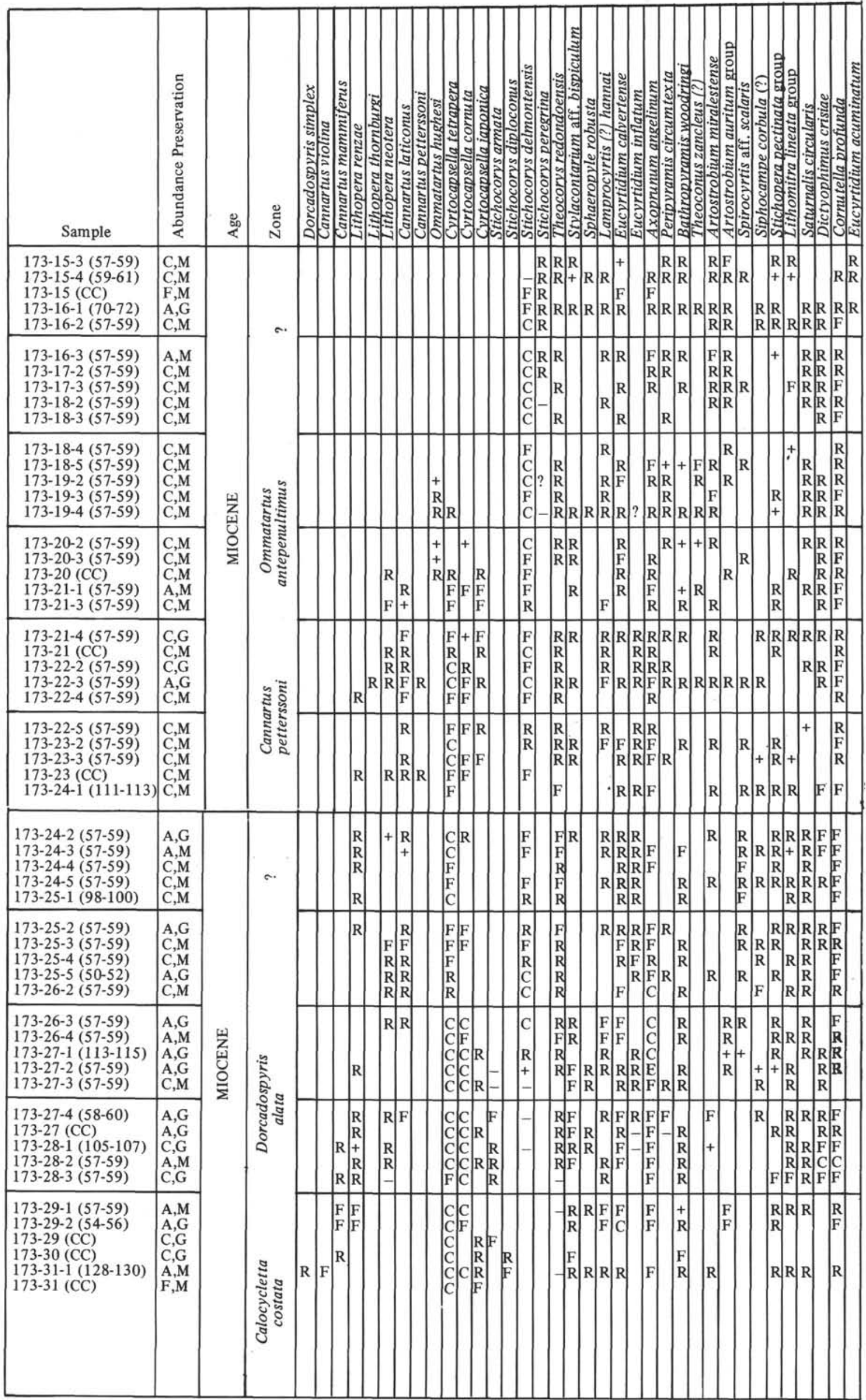




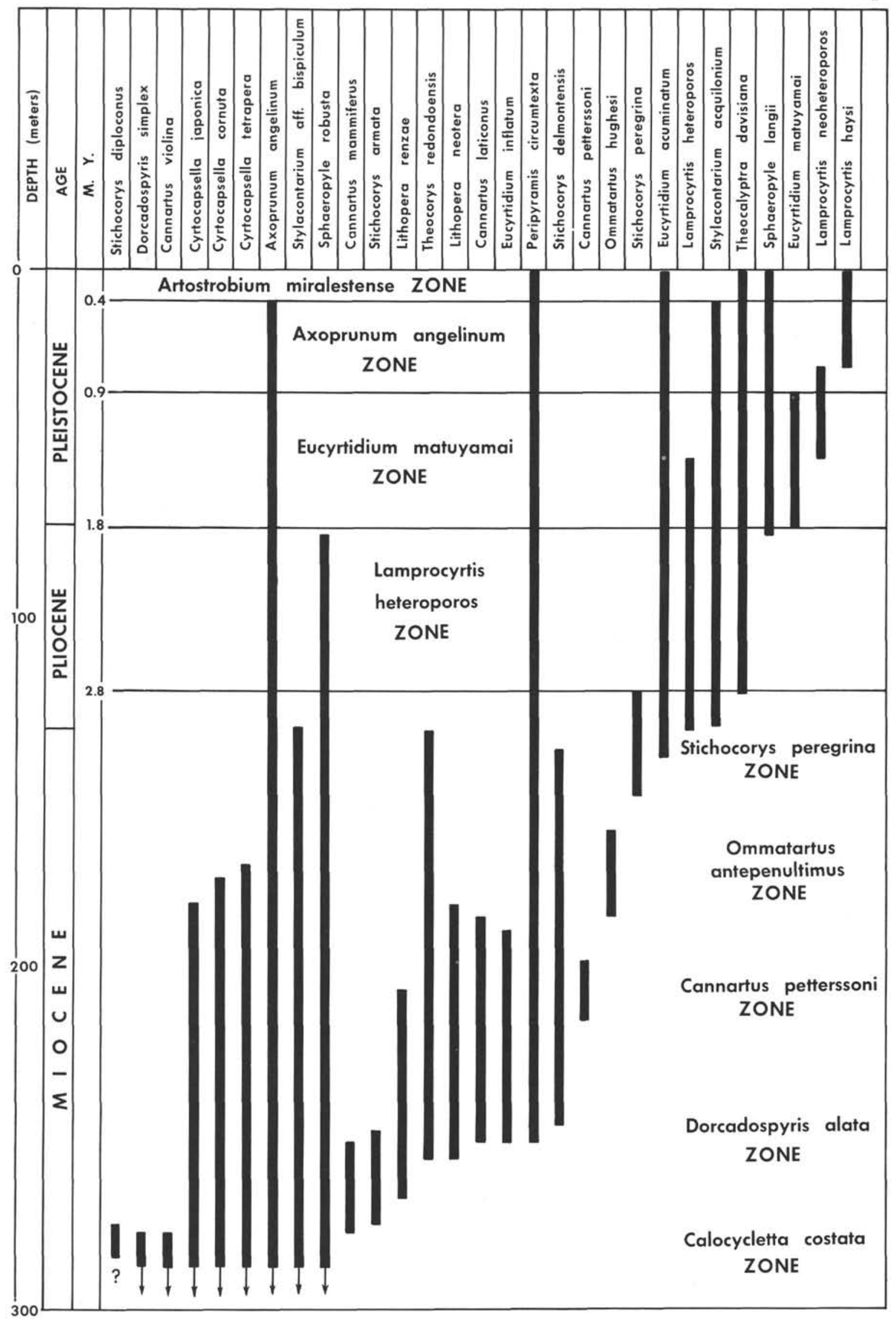

Figure 1. Species ranges at Site 173. 
the Axoprunum angelinum (= Stylatractus universus) Zone for these samples. The deepest Cenozoic radiolarian-bearing core is $174 \mathrm{~A}-35$ at about 380 meters below the sea floor.

Core 39 (Hole 174A) contains fairly large numbers of recrystallized radiolarians. This type of poor preservation is normally encountered in this region only in pre-Tertiary rocks, but no known Mesozoic forms were found.

Site $175\left(44^{\circ} 50^{\prime} \mathrm{N}, 125^{\circ} 15^{\prime} \mathrm{W}\right.$; water depth $\left.1999 \mathrm{~m}\right)$ (Table 3). Radiolarians occur in Cores 1 through 18 and in Core 20 , from the sea floor to a depth of about 185 meters. Preservation is moderate to good. Although radiolarians are seldom abundant, most samples contain enough to permit recognition of Pleistocene zones on the basis of upper limits of Axoprunum angelinum (= Stylatractus universus) and Stylacontarium acquilonium. Reworking is indicated by sporadic occurrences of Lamprocyrtis heteroporos, Stichocorys(?) sp., and Eucyrtidium matuyamai.

Site $176\left(45^{\circ} 56^{\prime} \mathrm{N}, 124^{\circ} 37^{\prime} \mathrm{W}\right.$; water depth $\left.193 \mathrm{~m}\right)$ (Table 4). Radiolarians are rare and moderately well preserved in Cores 1 through 5 , from the sea floor to a depth of about 42 meters. Zones cannot be recognized, but the presence of Lamprocyrtis heteroporos in 176-5-3 suggests a Pliocene age for the base of the sequence.

Site $177\left(50^{\circ} 28^{\prime} \mathrm{N}, 130^{\circ} 12^{\prime} \mathrm{W}\right.$; water depth $\left.2006 \mathrm{~m}\right)$ (Table 5). Radiolarians are rare to common in Cores 1 through 11, from the sea floor to a depth of about 127 meters, and preservation is moderate to good. Cores 12 through 26 are barren.

Absence of Axoprunum angelinum (= Stylatractus universus), in part of the sequence above the top of

TABLE 3

Radiolarians at Site 175

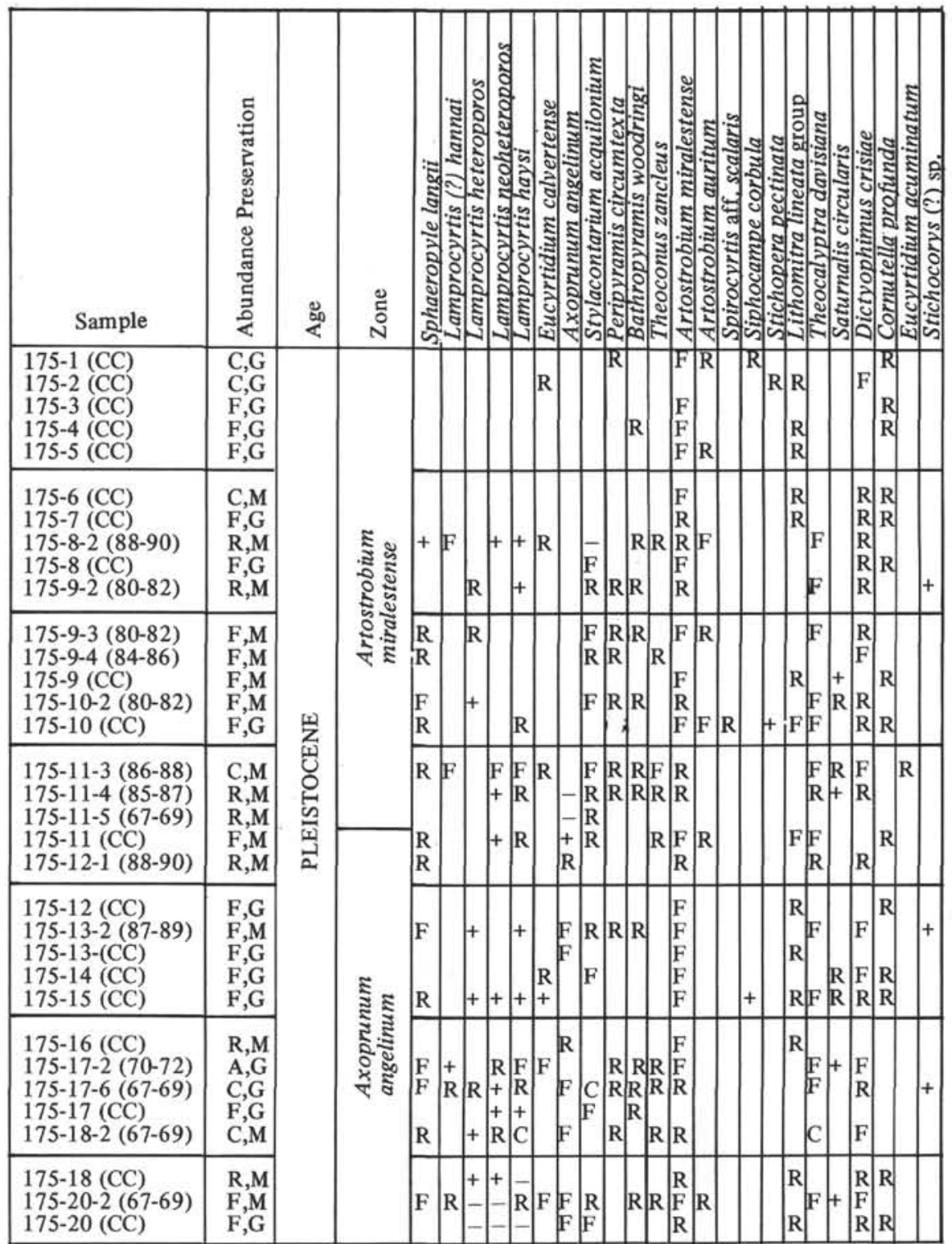


TABLE 4

Radiolarians at Site 176

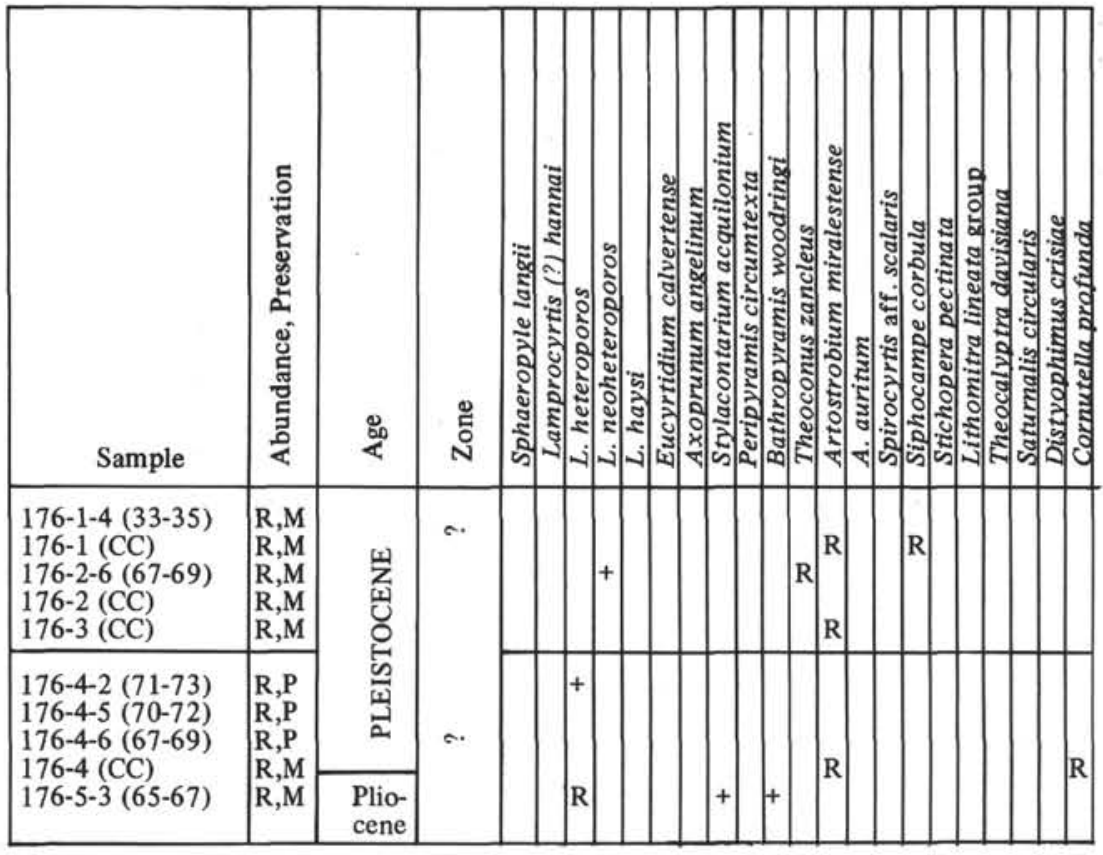

Eucyrtidium matuyamai suggests that the possible absence of the Axoprunum angelinum Zone. The E. matuyamai Zone is relatively thin. These observations suggest a possible hiatus in the depth range of approximately Cores 4 and 5, representing a minimum age span of 0.4 to 0.9 m.y.

Site $178\left(56^{\circ} 57^{\prime} \mathrm{N}, 147^{\circ} 08^{\prime} \mathrm{W}\right.$; water depth $\left.4218 \mathrm{~m}\right)$ (Table 6). Radiolarians occur in Cores 1 through 22 (sea floor to about 195 meters) in fairly large numbers, while Cores 23 through 54 (about 195 to 749 meters) contain sporadic radiolarians with few or no age-diagnostic species. Cores 55 through 59 (to a total depth of about 795 meters) contain no radiolarians. Pleistocene zones are recognizable in Cores 1 through 22 but Pliocene and older zones are not.

Site $179\left(56^{\circ} 25^{\prime} \mathrm{N}, 145^{\circ} 59^{\prime} \mathrm{W}\right.$; water depth $\left.3781 \mathrm{~m}\right)$ (Table 7). Radiolarians occur in Cores 1 through 10 (from the sea floor to about 89 meters) with variable abundance and preservation. Pleistocene zones can be tentatively recognized, but reworking of Miocene species is common. Because of the lack of the common Pliocene species Lamprocyrtis heteroporos, it is suggested that an exposure of Miocene sediment contributed the reworked material. Tentative Pleistocene events are based on intervals where reworked specimens are absent, but because the intervals are short and the reworked specimens seldom very abundant, the exact position of zone boundaries remains somewhat doubtful.

Site 180 . $\left(57^{\circ} 22^{\prime} \mathrm{N}, 147^{\circ} 51^{\prime} \mathrm{W}\right.$; water depth $\left.4923 \mathrm{~m}\right)$. Radiolarian occurrences are sporadic throughout Hole 180 from the sea floor to a depth of about 470 meters. Assemblages include a variety of extant species without diagnostic extinct species and probably represent the upper Pleistocene to Holocene Artostrobium miralestense (= Eucyrtidium tumidulum) Zone. Table 8 lists the species identified in the various samples.
Site 181. $\left(57^{\circ} 26^{\prime} \mathrm{N}, 148^{\circ} 28^{\prime} \mathrm{W}\right.$; water depth $\left.3086 \mathrm{~m}\right)$ Radiolarians occur sporadically in cores down to Core 18 (sea floor to about 169 meters) with abundant, diverse faunas in Cores 1, 17, and 18. Assemblages contain extant species with no species of zones below the upper Pleistocene-Holocene Artostrobium miralestense (= Eucyrtidium tumidulum) Zone. Samples 181-13-2(51-53 cm), 181-18. $2(41-43 \mathrm{~cm}), 181-18-2(54-59 \mathrm{~cm})$, and $181-18-2(93-95 \mathrm{~cm})$ contain one to three specimens each of Stylacontarium acquilonium (Hays) suggesting that the samples are in the lower part of this zone ( 0.3 to 0.4 m.y.). Species identified at this site are listed in Table 8.

Site 182. ( $57^{\circ} 53^{\prime} \mathrm{N}, 148^{\circ} 43^{\prime} \mathrm{W}$; water depths 1419 and $1434 \mathrm{~m}$ ) Radiolarians are rare to few and moderately well preserved in most cores from this site (sea floor to about 117 meters).

Core 1 of Hole 182 contains several extant species without species diagnostic of zones older than the upper Pleistocene-Holocene Artostrobium miralestense (= Eucyrtidium tumidulum) Zone. Core 2 contains very little fine material and was not examined. Core 3 contains Stylacontarium acquilonium and Axoprunum angelinum (= Stylatractus universus Hays), which represent the next lower $A$. angelinum zone.

Cores below Core 3 of Hole 182 and the single sample from Hole 182A lack age-diagnostic species. Species identified at this site are listed in Table 8 .

\section{RADIOLARIAN EVENTS AND POTENTIAL DATUM LEVELS}

Table 9 summarizes the sequence of events at the various sites for which adequate stratigraphic coverage is available, standardized to the order at Site 173. The list includes only those events which appear to be of potential stratigraphic 
TABLE 5

Radiolarians at Site 177

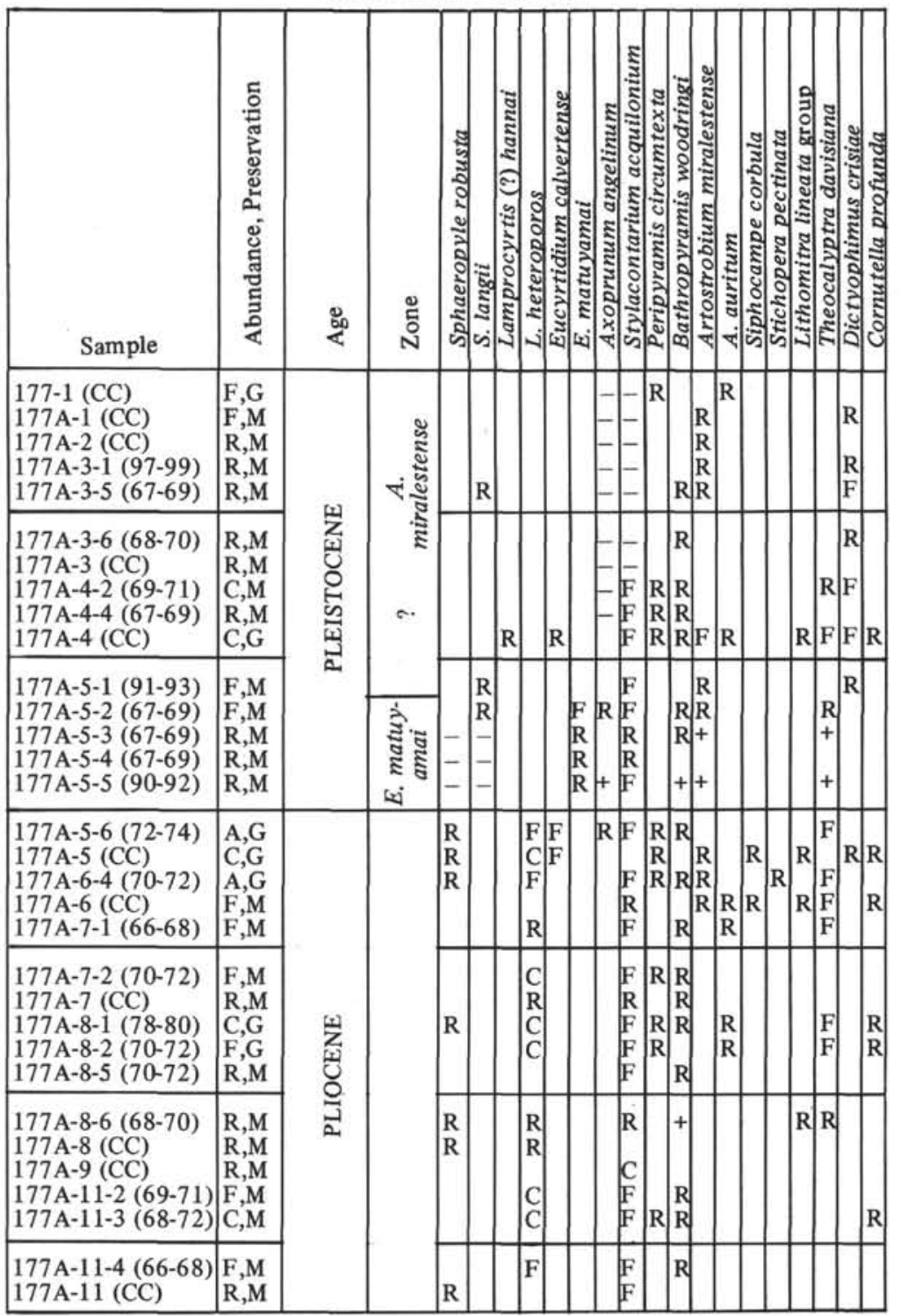

significance. Species with rare and sporadic occurrences thought to have been locally longer ranges were not included even though Table 2 may suggest a local event. In the left column, bottoms of ranges are indicated by B and tops by $\mathrm{T}$. Limits of ranges are identified as to whether they are considered morphotypic (m), evolutionary (e, includes extinctions), or morphotypic-evolutionary (m-e) according to the scheme proposed by Riedel and Sanfilippo (1971) and Sanfilippo and Riedel (in press). An arrow indicates an evolutionary transition. In the right columns, the core sections between which the events occurred at each site are noted followed, in parentheses, by the depth below the sea floor in meters. Reliability of the event levels, judged primarily from frequency of occurrence of the species in samples, and from abundance and preservation, is indicated as good $(\mathrm{G})$, moderate $(\mathrm{M})$, or poor $(\mathrm{P})$.

Comparison among the sites serves as a basis for correlation such that the depths in each column increase downward if the sequence is the same as that at Site 173 .
No inconsistencies are noted, so that the available stratigraphially important species appear to be reliable over the area covered by Leg 18 . However, the coincidence at Site 177 of the tops of the Axoprunum angelinum and Eucyrtidium matuyamai ranges suggests condensation of a part of the section represented by at least the $A$. angelinum (= Stylatractus universus) Zone and possibly part of the $E$. matuyamai Zone and involving a minimum time span of 0.4 to 0.9 m.y.

In addition to previously reported datum levels, several events that may be of stratigraphic significance (possibly only local) are worth noting here (see also Figure 1).

1) Bottom of Peripyramis circumtexta. This species is consistently present through much of the section, suggesting that its lower limit in the lower Miocene may prove useful in future correlations.

2) Bottom of Eucyrtidium acuminatum. This species is sporadically present but was not observed in its strict form (see Plate 10) below the upper Miocene. 
TABLE 6

Radiolarians at Site 178

\begin{tabular}{|c|c|c|c|c|c|c|c|c|c|c|c|}
\hline Sample & 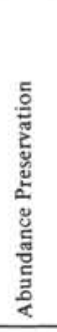 & $\stackrel{\text { 总 }}{<}$ & ్ㅗ․ & 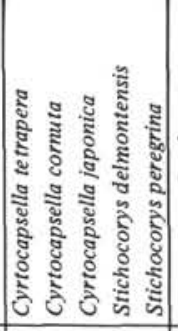 & 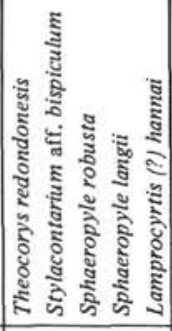 & 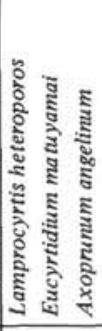 & 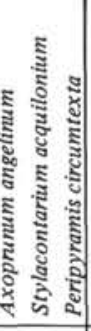 & 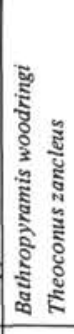 & 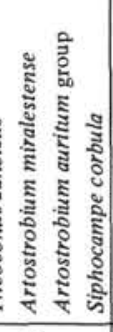 & 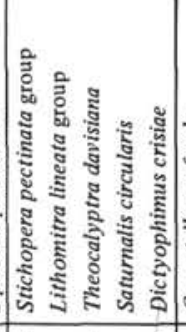 & \\
\hline $\begin{array}{l}178-1-4(86-88) \\
178-2 \text { (CC) } \\
178-3-4(61-63) \\
178-4-4(70-72) \\
178-5-1(110-112)\end{array}$ & $\begin{array}{l}\mathrm{C}, \mathrm{M} \\
\mathrm{A}, \mathrm{G} \\
\mathrm{C}, \mathrm{M} \\
\mathrm{C}, \mathrm{M} \\
\mathrm{R}, \mathrm{M}\end{array}$ & \multirow{12}{*}{ 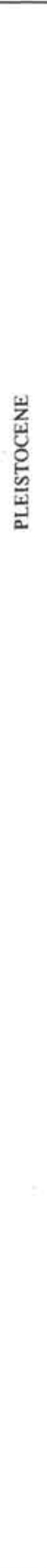 } & \multirow{6}{*}{ 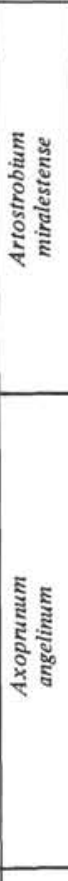 } & & $\begin{array}{l}\mathbf{R} \\
\mathbf{R} \\
\mathbf{R} \\
+ \\
\end{array}$ & $\begin{array}{l}- \\
- \\
- \\
- \\
-\end{array}$ & $\begin{array}{l}--\mathrm{R} \\
--\mathrm{R} \\
--\mathrm{R} \\
--\mathrm{R} \\
-- \\
\end{array}$ & $\mathrm{R}$ & $\begin{array}{ll}\mathrm{F} & \\
\mathrm{F} & \mathrm{R} \\
\mathrm{R} & \end{array}$ & 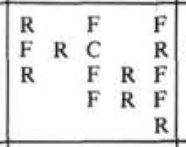 & R \\
\hline $\begin{array}{l}178-6-2 \text { (68-70) } \\
178-6-4 \text { (TOP) } \\
178-6-(\mathrm{CC}) \\
178-7 \text { (CC) } \\
178-8-1 \text { (90-92) }\end{array}$ & $\begin{array}{l}\mathrm{F}, \mathrm{M} \\
\mathrm{F}, \mathrm{M} \\
\mathrm{F}, \mathrm{M} \\
\mathrm{F}, \mathrm{M} \\
\mathrm{C}, \mathrm{M}\end{array}$ & & & & $\mathbf{R}$ & & $\begin{array}{l}--\bar{R} \\
-- \\
-\bar{R} \\
-R \\
-R\end{array}$ & & $\begin{array}{l}\mathrm{R} \\
\mathrm{F} \\
\mathrm{C}\end{array}$ & $\begin{array}{ccccc} & & & R \\
& F & F & R & R \\
R & R & F & R & R \\
& F & F & & R \\
& & F & R & F\end{array}$ & $\begin{array}{l}\mathrm{R} \\
\mathrm{R} \\
\mathrm{R} \\
\mathrm{R} \\
\mathrm{R}\end{array}$ \\
\hline $\begin{array}{l}178-8-3(67-69) \\
178-8-4(70-72) \\
178-8 \text { (CC) } \\
178-9-1(136-138) \\
178-9-2(65-67)\end{array}$ & $\begin{array}{l}\mathrm{R}, \mathrm{M} \\
\mathrm{R}, \mathrm{M} \\
\mathrm{R}, \mathrm{M} \\
\mathrm{R}, \mathrm{M}\end{array}$ & & & & & & $\begin{array}{l}R \\
R \\
-F \\
+\quad F \\
-\quad R \\
\end{array}$ & \multicolumn{2}{|l|}{$\mathrm{R}$} & + & \\
\hline $\begin{array}{l}178-9-3(67-69) \\
178-9-4(65-67) \\
178-9 \text { (CC) } \\
178-10 \text { (CC) } \\
178-11 \text { (CC) }\end{array}$ & $\begin{array}{l}\text { R,M } \\
\text { C,G } \\
\text { F,M } \\
\text { F,G } \\
\text { C,G }\end{array}$ & & & & & & $\begin{array}{lll}\mathrm{R} & \mathrm{F} & \mathrm{R} \\
\mathrm{F} & \mathrm{C} & \mathrm{R} \\
\mathrm{F} & \mathrm{F} & \\
\mathrm{F} & \mathrm{F} & \\
\mathrm{F} & \mathrm{F} & \mathrm{R}\end{array}$ & $\begin{array}{ll}\mathrm{R} & \mathrm{R} \\
\mathrm{R}\end{array}$ & $\begin{array}{l}\mathrm{F} \\
\mathrm{F} \\
\mathrm{F}\end{array}$ & \begin{tabular}{llll|} 
& $F$ & $R$ & $F$ \\
$R$ & & $F$ \\
$R$ & $F$ & & $F$ \\
$R$ & $F$ & & $F$ \\
\end{tabular} & R \\
\hline $\begin{array}{l}178-12-1(119-121) \\
178-12-2(92-94) \\
178-12-3(117-119) \\
178-12(C C) \\
178-13-1(109-111)\end{array}$ & $\begin{array}{l}\mathrm{R}, \mathrm{M} \\
\mathrm{R}, \mathrm{M} \\
\mathrm{F}, \mathrm{M} \\
\mathrm{F}, \mathrm{G} \\
\mathrm{C}, \mathrm{G}\end{array}$ & & & & & & 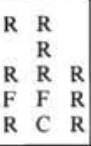 & & $\begin{array}{lll}R & \\
R & R \\
R & R \\
F & R & \end{array}$ & 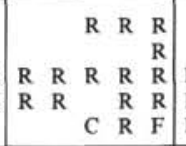 & $\begin{array}{l}\mathrm{R} \\
\mathrm{R} \\
\mathrm{R} \\
\end{array}$ \\
\hline $\begin{array}{l}178-13-2(92-94) \\
178-13-3(67-69) \\
178-13 \text { (CC) } \\
178-14 \text { (CC) } \\
178-15-1(90-92)\end{array}$ & $\begin{array}{l}\mathrm{R}, \mathrm{M} \\
\mathrm{R}, \mathrm{M} \\
\mathrm{F}, \mathrm{M} \\
\mathrm{R}, \mathrm{M} \\
\mathrm{F}, \mathrm{M}\end{array}$ & & & & & $\begin{array}{r}\mathrm{R} \\
+\quad \\
\end{array}$ & $\begin{array}{ll} & \mathrm{R} \\
\mathrm{R} \\
\mathrm{R} \\
\mathrm{R} \\
\mathrm{F} \\
\mathrm{R} & \\
\mathrm{C} & \mathrm{R}\end{array}$ & & & $\begin{array}{lll}R & \\
& R & \\
& R\end{array}$ & R \\
\hline $\begin{array}{l}178-15-2(67-69) \\
178-15(\mathrm{CC}) \\
178-16-2(75-77) \\
178-16-3(69-70) \\
178-16-4(67-69)\end{array}$ & $\begin{array}{l}\mathrm{A}, \mathrm{G} \\
\mathrm{R}, \mathrm{M} \\
\mathrm{R}, \mathrm{M} \\
\mathrm{R}, \mathrm{M} \\
\mathrm{C}, \mathrm{M}\end{array}$ & & \multirow{6}{*}{ 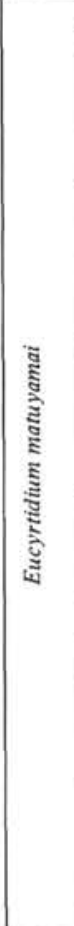 } & & $\mathrm{R}$ & $\begin{array}{l}\mathrm{F} \\
\mathrm{R}\end{array}$ & $\begin{array}{ll}\mathrm{C} & \mathrm{R} \\
\mathrm{R} & \\
\mathrm{R} & \\
\mathrm{F} & \\
\mathrm{C} & \end{array}$ & $\begin{array}{l}\mathrm{R} \\
+ \\
\mathrm{R}\end{array}$ & $\begin{array}{l}\text { F } \\
\text { F }\end{array}$ & $\begin{array}{lll}\mathrm{F} & \mathrm{R} & \mathrm{F} \\
& & \\
\mathrm{R} & \mathrm{R}\end{array}$ & R \\
\hline $\begin{array}{l}178-16 \text { (CC) } \\
178-17-1(87-89) \\
178-17-2(83-85) \\
178-17-3(83-84) \\
178-17-4(73-75)\end{array}$ & $\begin{array}{l}\mathrm{F}, \mathrm{M} \\
\mathrm{R}, \mathrm{M} \\
\mathrm{R}, \mathrm{M} \\
\mathrm{R}, \mathrm{M} \\
\mathrm{R}, \mathrm{M}\end{array}$ & & & & & & $\begin{array}{l}\mathrm{F} \\
\mathrm{R} \\
\mathrm{R} \\
\mathrm{R} \\
\mathrm{R}\end{array}$ & + & & & \\
\hline $\begin{array}{l}: 78-17-5(73-75) \\
178-17 \text { (CC) } \\
178-18-1(134-136) \\
178-18-2(84-86) \\
178-18-3(65-67)\end{array}$ & $\begin{array}{l}\text { R,M } \\
\text { F,M } \\
\text { A,G } \\
\text { R,M } \\
\text { C,G }\end{array}$ & & & & $\mathrm{R}$ & $\begin{array}{ll}R & R \\
& R \\
F & F\end{array}$ & $\begin{array}{lll} & F & \\
& F & \\
R & C & R \\
R & & \\
F & C & R\end{array}$ & $\begin{array}{l}+ \\
R \\
R\end{array}$ & $\begin{array}{ll}F & R \\
C & F\end{array}$ & \begin{tabular}{ll|}
$R$ & $F$ \\
& $F$
\end{tabular} & \\
\hline $\begin{array}{l}178-18 \text { (CC) } \\
178-19-1(67-69) \\
178-19-2(84-86) \\
178-19-3(67-69) \\
178-19 \text { (CC) }\end{array}$ & $\begin{array}{l}\mathrm{F}, \mathrm{M} \\
\mathrm{F}, \mathrm{M} \\
\mathrm{R}, \mathrm{M} \\
\mathrm{C}, \mathrm{G} \\
\mathrm{R}, \mathrm{M}\end{array}$ & & & & & $\begin{array}{ll} & \mathrm{F} \\
& \mathrm{R} \\
\mathrm{R} & \mathrm{R} \\
\mathrm{R} & \mathrm{R}\end{array}$ & 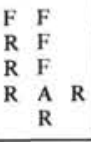 & $\begin{array}{l}\mathrm{R} \\
\mathrm{F} \\
\mathrm{F}\end{array}$ & c & \begin{tabular}{lll|}
$R$ & $F$ & $R$ \\
& & $F$ \\
$R$ & & \\
\end{tabular} & F \\
\hline $\begin{array}{l}178-20-2(67-69) \\
178-20-3(67-69) \\
178-20-4(61-63) \\
178-20-5(67-69) \\
178-20-6(66-68)\end{array}$ & $\begin{array}{l}\mathrm{R}, \mathrm{M} \\
\mathrm{C}, \mathrm{G} \\
\mathrm{C}, \mathrm{G} \\
\mathrm{R}, \mathrm{M} \\
\mathrm{R}, \mathrm{M}\end{array}$ & & & & & $\begin{array}{ll}\mathrm{F} & \mathrm{F} \\
\mathrm{F} & \mathrm{R} \\
& \end{array}$ & $\begin{array}{ll} & \mathrm{R} \\
\mathrm{F} & \mathrm{A} \\
\mathrm{R} & \mathrm{A} \\
\mathrm{R} \\
\mathrm{R} \\
\mathrm{R}\end{array}$ & $\begin{array}{l}\mathrm{F} \\
\mathrm{R} \\
+\end{array}$ & $\begin{array}{l}\mathrm{C} \\
\mathrm{F}\end{array}$ & $\begin{array}{ll}\mathbf{R} & \mathbf{R} \\
& \mathrm{F}\end{array}$ & \\
\hline $\begin{array}{l}178-20 \text { (CC) } \\
178-21-1(143-145) \\
178-21-2(67-69) \\
178-21-3(68-70) \\
178-21-4(67-69)\end{array}$ & $\begin{array}{l}\mathrm{R}, \mathrm{P} \\
\mathrm{R}, \mathrm{M} \\
\mathrm{R}, \mathrm{M} \\
\mathrm{R}, \mathrm{M} \\
\mathrm{R}, \mathrm{M}\end{array}$ & & & & & + & $\begin{array}{ll}\mathrm{F} & \\
\mathrm{F} & \\
\mathrm{F} & \\
\mathrm{F} & \\
\mathrm{C} & \mathrm{R}\end{array}$ & + & & R & \\
\hline
\end{tabular}


TABLE 6 - Continued

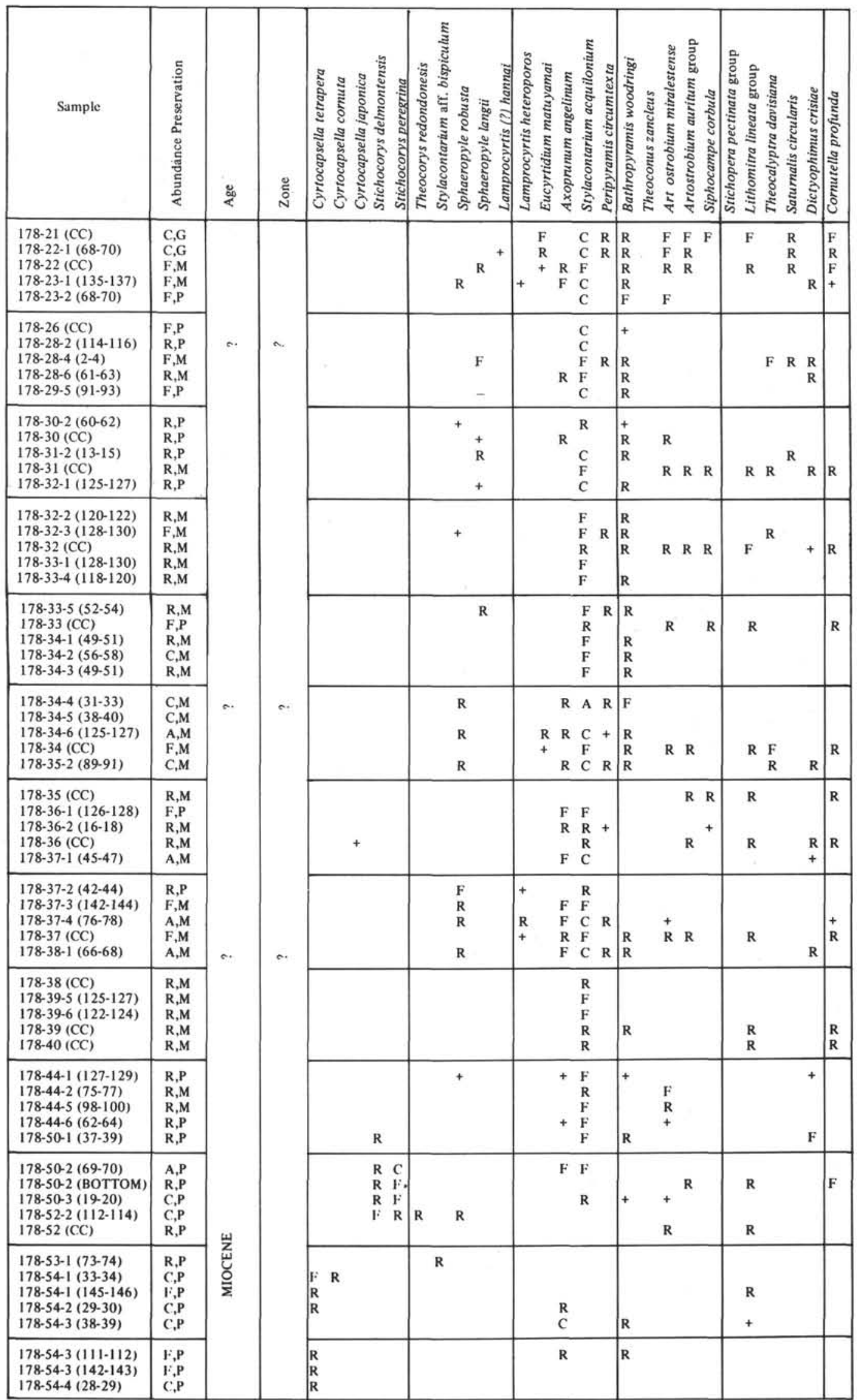


TABLE 7

Radiolarians at Site 179

\begin{tabular}{|c|c|c|c|c|c|c|c|c|c|c|c|c|c|c|c|c|c|c|c|c|c|c|}
\hline Sample & 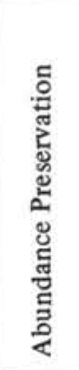 & $\stackrel{\sharp}{<}$ & ֻั & 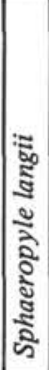 & 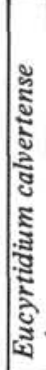 & 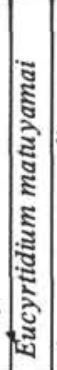 & 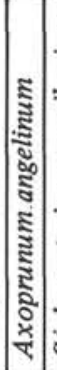 & 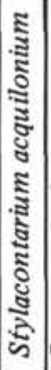 & 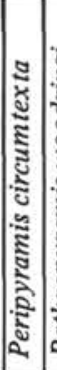 & 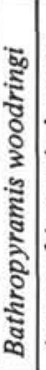 & 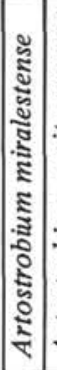 & 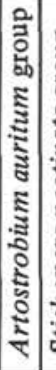 & 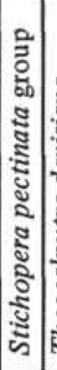 & 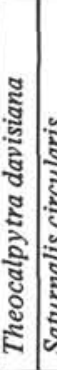 & 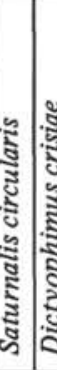 & 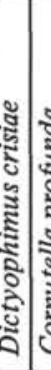 & 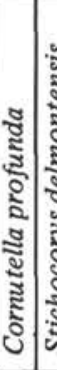 & 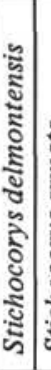 & 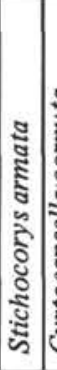 & 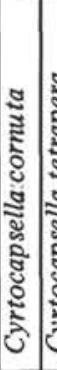 & 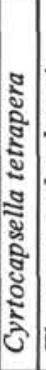 & 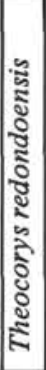 \\
\hline $\begin{array}{l}179-1-1(133-135) \\
179-1-2(98-100) \\
179-1-3(118-120) \\
179-1(C C) \\
179-2-1(106-108)\end{array}$ & $\begin{array}{l}\mathrm{R}, \mathrm{M} \\
\mathrm{R}, \mathrm{P} \\
\mathrm{C}, \mathrm{M} \\
\mathrm{C}, \mathrm{G} \\
\mathrm{F}, \mathrm{M}\end{array}$ & \multirow{10}{*}{ 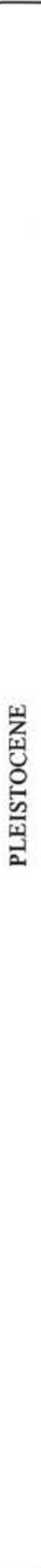 } & \multirow{4}{*}{ 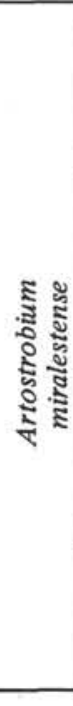 } & $\mathrm{R}$ & & & & & $\begin{array}{l}\mathrm{R} \\
\mathrm{R} \\
\mathrm{R} \\
\mathrm{R} \\
\mathrm{R}\end{array}$ & & $\mathrm{F}$ & & & $\begin{array}{l}\mathrm{R} \\
\mathrm{F} \\
\mathrm{F}\end{array}$ & & $\begin{array}{l}F \\
F \\
F\end{array}$ & $\mathbf{R}$ & & & & & \\
\hline $\begin{array}{l}179-2-2(10-12) \\
179-2-3(119-121) \\
179-2-4(61-63) \\
179-2-5(96-98) \\
179-2 \text { (CC) }\end{array}$ & $\begin{array}{l}\text { R,P } \\
\text { R,M } \\
\text { A,G } \\
\text { C,M } \\
\text { F,G }\end{array}$ & & & F & & & $\begin{array}{l}\mathrm{C} \\
\mathrm{R}\end{array}$ & \begin{tabular}{|l|} 
\\
$\mathrm{R}$ \\
$\mathrm{R}$ \\
$\mathrm{F}$
\end{tabular} & \begin{tabular}{l|}
$\mathrm{R}$ \\
$\mathrm{R}$ \\
$\mathrm{R}$
\end{tabular} & $\begin{array}{l}\mathrm{R} \\
\mathrm{R}\end{array}$ & F & $\begin{array}{l}+ \\
\mathrm{R}\end{array}$ & $\mathrm{R}$ & R $\mathrm{F}$ & R & \begin{tabular}{l|l}
$\mathrm{R}$ & \\
$\mathrm{R}$ &
\end{tabular} & $\mathrm{R}$ & $\mathrm{R}$ & $\mathrm{R}$ & C & $\begin{array}{l}\mathrm{R} \\
\mathrm{C} \\
\mathrm{R} \\
\mathrm{R}\end{array}$ & $\mathrm{R}$ \\
\hline $\begin{array}{l}179-3-1(110-112) \\
179-3-2(90-92) \\
179-3-3(90-92) \\
179-3-4(109-111) \\
179-3-5(29-31)\end{array}$ & $\begin{array}{l}\mathrm{C}, \mathrm{G} \\
\mathrm{R}, \mathrm{M} \\
\mathrm{R}, \mathrm{M} \\
\mathrm{R}, \mathrm{M} \\
\mathrm{R}, \mathrm{M}\end{array}$ & & & $\begin{array}{l}\mathrm{R} \\
\mathrm{R}\end{array}$ & & & $\begin{array}{l}- \\
- \\
- \\
- \\
-\end{array}$ & $\begin{array}{l}- \\
\mathrm{R} \\
\mathrm{R} \\
\mathrm{F} \\
\mathrm{C}\end{array}$ & R & $\mathrm{R}$ & $\mathbf{R}$ & $\mathbf{R}$ & $\mathrm{R}$ & $\begin{array}{l}\mathrm{F} \\
\mathrm{R}\end{array}$ & & $\begin{array}{l}F \\
R\end{array}$ & & & & & & \\
\hline $\begin{array}{l}179-3-6(141-143) \\
179-3(\mathrm{CC}) \\
179-4-1(127-129) \\
179-4-3(87-89) \\
179-4-4(83-85)\end{array}$ & $\begin{array}{l}\mathrm{R}, \mathrm{M} \\
\mathrm{R}, \mathrm{M} \\
\mathrm{R}, \mathrm{M} \\
\mathrm{R}, \mathrm{M} \\
\mathrm{C}, \mathrm{G}\end{array}$ & & & $\mathrm{R}$ & & & $\begin{array}{l}- \\
- \\
- \\
- \\
\mathrm{R}\end{array}$ & $\begin{array}{l}\mathrm{F} \\
\mathrm{F} \\
\mathrm{F} \\
\mathrm{C} \\
\mathrm{F}\end{array}$ & $\mathrm{R}$ & $\mathrm{R}$ & $\mathrm{R}$ & $\mathbf{R}$ & & $\mathrm{F}$ & \begin{tabular}{l|l}
+ & $\mathrm{R}$ \\
$\mathrm{F}$ & $\mathrm{F}$ \\
$\mathrm{R}$ & $\mathrm{F}$
\end{tabular} & $\begin{array}{l}\mathrm{R} \\
\mathrm{R} \\
\mathrm{F}\end{array}$ & & & & $\mathrm{R}$ & $\mathrm{F}$ & \\
\hline $\begin{array}{l}179-4 \text { (CC) } \\
179-5-1(37-39) \\
179-5-2(130-132) \\
179-5-3(46-48) \\
179-5-4(82-84)\end{array}$ & $\begin{array}{l}\text { F,M } \\
\text { R,M } \\
\text { A,G } \\
\text { R,M } \\
\text { R,M }\end{array}$ & & \multirow{2}{*}{ 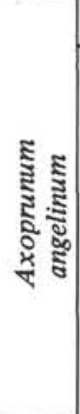 } & \begin{tabular}{|l|}
$\mathrm{R}$ \\
$\mathrm{R}$ \\
\end{tabular} & R & R & $\begin{array}{c}\mathrm{R} \\
\mathrm{F} \\
\mathrm{R} \\
+\end{array}$ & $\begin{array}{l}\mathrm{F} \\
\mathrm{F} \\
\mathrm{A} \\
\mathrm{F} \\
\mathrm{C}\end{array}$ & $\begin{array}{l}\mathrm{R} \\
+ \\
\mathrm{R}\end{array}$ & $\begin{array}{l}\mathrm{R} \\
\mathrm{R}\end{array}$ & $\begin{array}{l}\mathrm{F} \\
\mathrm{F}\end{array}$ & $\mathrm{R}$ & $\mathrm{R}$ & \begin{tabular}{l|l}
$\mathrm{F}$ & $\mathrm{F}$ \\
$\mathrm{R}$ &
\end{tabular} & $\mathrm{R}$ & \begin{tabular}{l|l}
$\mathrm{R}$ & \\
$\mathrm{F}$
\end{tabular} & $\mathrm{R}$. & + & & $\begin{array}{l}R \\
R\end{array}$ & $\begin{array}{l}+ \\
\mathrm{R} \\
\mathrm{F} \\
\mathrm{R}\end{array}$ & \\
\hline $\begin{array}{l}179-5-5(65-67) \\
179-5-5(125-127) \\
179-5(\mathrm{CC}) \\
179-6-1(130-132) \\
179-6-2(66-68)\end{array}$ & $\begin{array}{l}\mathrm{R}, \mathrm{M} \\
\mathrm{R}, \mathrm{M} \\
\mathrm{R}, \mathrm{M} \\
\mathrm{R}, \mathrm{M} \\
\mathrm{R}, \mathrm{M}\end{array}$ & & & $\mathrm{R}$ & & & \begin{tabular}{|l|}
$\mathrm{R}$ \\
$\mathrm{R}$ \\
$\mathrm{R}$
\end{tabular} & $\begin{array}{l}\mathrm{R} \\
\mathrm{F} \\
\mathrm{F} \\
\mathrm{F} \\
\mathrm{F}\end{array}$ & & & $\mathrm{R}$ & & & $\begin{array}{l}+ \\
\mathrm{F} \\
+ \\
\mathrm{R}\end{array}$ & & $\begin{array}{l}+ \\
\mathrm{F}\end{array}$ & $\mathrm{R}$ & $\mathrm{R}$ & & $\mathbf{R}$ & $\mathrm{R}$ & \\
\hline $\begin{array}{l}179-6(\mathrm{CC}) \\
179-7-2(70-72) \\
179-7-3(62-64) \\
179-7-4(55-57) \\
179-7-5(108-110)\end{array}$ & $\begin{array}{l}\text { C,M } \\
\text { R,P } \\
\text { R,P } \\
\text { F,M } \\
\text { F,M }\end{array}$ & & \multirow{4}{*}{ 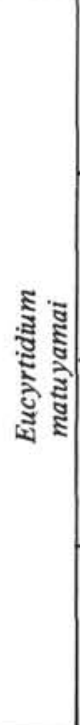 } & $\begin{array}{l}\mathrm{R} \\
\mathrm{R}\end{array}$ & + & $\mathrm{R}$ & $\begin{array}{l}\mathrm{R} \\
+ \\
\mathrm{R}\end{array}$ & $\begin{array}{l}\mathrm{F} \\
\mathrm{R} \\
\mathrm{R} \\
\mathrm{F} \\
\mathrm{C}\end{array}$ & $\begin{array}{l}\mathrm{R} \\
+ \\
\mathrm{R} \\
\mathrm{R}\end{array}$ & $\mathbf{R}$ & $\mathbf{R}$ & + & & $\mathbf{R} \mid \begin{array}{l}\mathbf{1} \\
\mathrm{r} \\
\mathrm{I}\end{array}$ & \begin{tabular}{l|l}
$R$ & $F$ \\
+ & \\
$R$ & + \\
$R$ &
\end{tabular} & \begin{tabular}{l|l}
$\mathrm{R}$ & 1. \\
+ &
\end{tabular} & \begin{tabular}{l|l}
$\mathrm{R}$ & 1 \\
+ & \\
$\mathrm{R}$ &
\end{tabular} & $\mathrm{R}$ & & & & \\
\hline $\begin{array}{l}179-7 \text { (CC) } \\
179-8-4(17-19) \\
179-8(C C) \\
179-9-1(30-32) \\
179-9-2(70-72)\end{array}$ & $\begin{array}{l}\mathrm{F}, \mathrm{M} \\
\mathrm{R}, \mathrm{M} \\
\mathrm{F}, \mathrm{M} \\
\mathrm{F}, \mathrm{M} \\
\mathrm{R}, \mathrm{M}\end{array}$ & & & $\mathrm{R}$ & & $\begin{array}{l}\mathrm{R} \\
\mathrm{R} \\
\mathrm{R} \\
+\end{array}$ & \begin{tabular}{|l|}
$R$ \\
$R$ \\
$R$ \\
+ \\
\end{tabular} & $\begin{array}{l}\mathrm{F} \\
\mathrm{F} \\
\mathrm{F} \\
\mathrm{C} \\
\mathrm{F}\end{array}$ & \begin{tabular}{|l|}
$\mathrm{R}$ \\
$\mathrm{R}$ \\
$\mathrm{R}$
\end{tabular} & $\begin{array}{l}\mathrm{R} \\
\mathrm{R} \\
\mathrm{R} \\
\mathrm{R} \\
\mathrm{R}\end{array}$ & $\begin{array}{l}\mathrm{F} \\
\mathrm{R} \\
\mathrm{R}\end{array}$ & $\mathrm{R}$ & & $\begin{array}{l}\mathrm{R} \\
\mathrm{R}\end{array}$ & $\begin{array}{l}\mathrm{R} \\
\mathrm{F}\end{array}$ & & $\begin{array}{l}\mathrm{R} \\
\mathrm{R} \\
\mathrm{F}\end{array}$ & $\mathrm{R}$ & & & & + \\
\hline $\begin{array}{l}179-9-3(14-16) \\
179-9-4(102-104) \\
179-9-5(60-62) \\
179-9-6(48-50) \\
179-9(\mathrm{CC})\end{array}$ & $\begin{array}{l}\mathrm{R}, \mathrm{M} \\
\mathrm{R}, \mathrm{M} \\
\mathrm{F}, \mathrm{M} \\
\mathrm{R}, \mathrm{M} \\
\mathrm{F}, \mathrm{M}\end{array}$ & & & $\begin{array}{l}\mathrm{R} \\
\mathrm{R}\end{array}$ & & $\begin{array}{l}+ \\
+ \\
\mathrm{R}\end{array}$ & $\mathrm{R}$ & $\begin{array}{l}\mathrm{R} \\
\mathrm{F} \\
\mathrm{C} \\
\mathrm{R} \\
\mathrm{F} \\
\end{array}$ & & $\begin{array}{l}\mathrm{R} \\
\mathrm{R} \\
\mathrm{R} \\
\mathrm{R} \\
\end{array}$ & $\mathbf{R}$ & & & $\mathrm{R}$ & & & & $\mathrm{R}$ & & & & \\
\hline $\begin{array}{l}179-10-1(114-116) \\
179-10-2(122-124) \\
179-10-3(120-122) \\
179-10-4(65-67) \\
179-10 \text { (CC) }\end{array}$ & $\begin{array}{l}\mathrm{R}, \mathrm{M} \\
\mathrm{R}, \mathrm{M} \\
\mathrm{R}, \mathrm{M} \\
\mathrm{R}, \mathrm{M} \\
\mathrm{R}, \mathrm{M}\end{array}$ & & & & R & & R & $\begin{array}{l}\text { F } \\
C \\
C \\
F \\
F\end{array}$ & & $\begin{array}{l}\mathrm{R} \\
\mathrm{R} \\
\mathrm{R} \\
\mathrm{R} \\
\mathrm{R}\end{array}$ & & $\mathbf{R}$ & & & & & & $\mathrm{R}$ & + & & & + \\
\hline
\end{tabular}


TABLE 8

Species Identified at Sites 180, 181, and 182, (X Indicates Presence)

\begin{tabular}{lccc}
\hline & 180 & 181 & 182 \\
\hline Stylacontarium acquilonium & & $\mathrm{X}$ & $\mathrm{X}$ \\
Saturnalis circularis & & $\mathrm{X}$ & \\
Sphaeropyle langii & & $\mathrm{X}$ & \\
Spongotrochus glacialis & & $\mathrm{X}$ & \\
Bathropyramis woodringi & & $\mathrm{X}$ & \\
Clathrocyclas spp. & $\mathrm{X}$ & $\mathrm{X}$ & $\mathrm{X}$ \\
Cornutella profunda & $\mathrm{X}$ & $\mathrm{X}$ & $\mathrm{X}$ \\
Dictyophimus crisiae & $\mathrm{X}$ & $\mathrm{X}$ & $\mathrm{X}$ \\
Peripyramis circumtexta & $\mathrm{X}$ & $\mathrm{X}$ & \\
Pterocanium korotnevi & $\mathrm{X}$ & $\mathrm{X}$ & \\
Pterocanium spp. & $\mathrm{X}$ & $\mathrm{X}$ & $\mathrm{X}$ \\
Stichopera pectinata & & $\mathrm{X}$ & \\
Theocorythium davisiana & $\mathrm{X}$ & $\mathrm{X}$ & \\
Artostrobium auritum group & $\mathrm{X}$ & $\mathrm{X}$ & $\mathrm{X}$ \\
Artostrobium miralestense & $\mathrm{X}$ & $\mathrm{X}$ & $\mathrm{X}$ \\
Lithomitra lineata group & $\mathrm{X}$ & $\mathrm{X}$ & \\
Siphocampe corbula & & & \\
\hline
\end{tabular}

3) Top of Theocorys redondoensis. This relatively common species disappears near or at the Miocene-Pliocene boundary both at Site 173 and probably in land sections (Casey et al., 1972; Casey, in press; unpublished data).

4) Bottom of Lamprocyrtis heteroporos. This level is tentatively taken as the Miocene-Pliocene boundary (see discussion in Section 2).

5) Stylacontarium sp. aff. S. bispiculum to $S$. acquilonium. This transition occurs near the Miocene-Pliocene boundary.

6) Bottom of Theocalyptra davisiana. This species first appears in the lower Pliocene.

7) Sphaeropyle robusta to $S$. langii. This transtion occurs near (possibly at) the Pliocene-Pleistocene boundary.

8) Lamprocyrtis heteroporos to L. neoheteroporos. This transition takes place in the lower Pleistocene Eucrytidium matuyamai Zone.

9) Lamprocyrtis neoheteroporos to L. haysi. This transition takes place in Pleistocene Axoprunum angelinum Zone.

\section{RANGE CHART AND GEOGRAPHIC VARIATION IN SPECIES RANGES}

Figure 1 summarizes the ranges of selected species at Site 173 , the only site with a long, continuous sequence of radiolarians. This chart may be applicable in other Pacific Coast (California Current) sections, but the ranges of some species are different from those previously reported in both equatorial and subarctic North Pacific regions. Apparently shortened ranges of equatorial species (e.g., the artiscins) are generally based on rare, discontinuous occurrences and probably represent occasional influxes of equatorial water. These permit correlations with equatorial sequences but are of little other biostratigraphic significance. In other cases, longer ranges of species indicate a previously unknown segment of the geologic history of a species. These are generally more important contributions to future biostratigraphic interpretations. Brief discussions of possibly significant local variations in ranges follow:
1) Stichocorys delmontensis. This species ranges well above the first appearance of its descendent species, Stichocorys peregrina, nearly to the Miocene-Pliocene boundary. Though this could possibly be interpreted as an overlap in morphotypic ranges (Riedel and Sanfilippo, 1970; Sanfilippo and Riedel, in press), they are thought by Riedel and Sanfilippo $(1970,1971)$ to be generally nonoverlapping. However, overlap and an apparently earlier appearance of $S$. peregrina were noted in the western (Kling, 1971) and equatorial (Goll, 1972) Pacific Ocean. Furthermore, a distinct change in dominance from one morphotype to the other is not evident (at Site 173), and the overlap covers a considerable stratigraphic interval.

2) Crytocapsella. Cyrtocapsella cornuta, C. tetrapera, and $C$. japonica appear to range higher here than in tropical regions. All three species overlap the range of Ommatartus hughesi, whereas they normally are thought not to by Riedel and Sanfilippo (1971). Furthermore, their order of disappearance is altered. All three species reach upper limits at about the same level, while $C$. tetrapera previously disappeared in the Dorcadospyris alata Zone and C. cornuta in the Cannartus(?) petterssoni Zone. C. japonica is less common and its range consequently less reliable; but it seldom ranges as high as $C$. tetrapera. However, the limits of these species have been regarded as morphotypic and thus. more subject to geographic variability. Inconsistencies in reported range of these species have also been noted by Goll (1972).

3) Lamprocyrtis heteroporos. This species reaches an upper limit at the Pliocene-Pleistocene boundary (first evolutionary appearance of Eucyrtidium matuyamai) both in DSDP Leg 18 northern sites $(177,178)$ and in previously reported subarctic cores (Hays, 1970; Kling, 1971). Here, however, it persists into the Pleistocene, overlaps $E$. matuyamai, and evolves into L. neoheteroporos.

4) Stylacontarium acquilonium. In addition to these longer ranges, one shortened range at Site 173 may be of significance. Stylacontarium acquilonium normally ranges somewhat above the upper limit of Axoprunum angelinum, but here the two species disappear at about the same level. Thus, it may not be possible here to determine the $0.4 \mathrm{~m} . \mathrm{y}$. level indicated by the upper limit of $S$. acquilonium farther north.

\section{EVOLUTIONARY LINEAGES}

Of particular importance for future biostratigraphic application of the information included in this report are several suggested evolutionary transitions. Some of these are new, while others are confirmations of trends previously reported in other areas.

1) Sphaeropyle robusta - Sphaeropyle langii (Sites 173, $177,178)$. S. robusta whose ancestor is unknown, appears to evolve into $S$. langii in the uppermost Pleistocene. $S$. langii persisted through the Pleistocene and is still living.

2) Eucyrtidium calvertense - Eucyrtidium inflatum (Site 173). $E$. inflatum evolves from $E$. calvertense in the middle(?) Miocene and reaches extinction without apparent descendents in the upper(?) Miocene.

3) Eucyrtidium calvertense - Eucyrtidium matuyamai (Sites 173, 177). This transition, which tends to occur on the Pliocene-Pleistocene boundary, first noted by Hays 
TABLE $9^{\text {a }}$

Radiolarian Events at Sites 173 and 175-178

\begin{tabular}{|c|c|c|c|c|c|c|c|c|c|c|c|}
\hline \multicolumn{3}{|c|}{ Radiolarian Events } & \multicolumn{2}{|l|}{ Site 173} & \multicolumn{2}{|l|}{ Site 175} & Śite 176 & Site $177 \mathrm{~A}$ & Site 178 & \multicolumn{2}{|c|}{ Site 179} \\
\hline $\mathrm{T}$ & $\begin{array}{l}\text { Stylacontarium } \\
\text { acquilonium }\end{array}$ & $\mathrm{m}$ & $\begin{array}{l}2-2 \\
2-3 \\
(7-9)\end{array}$ & $\mathbf{P}$ & $\begin{array}{l}8-2 \\
8(\mathrm{CC}) \\
(64-72)\end{array}$ & M & & $\begin{array}{l}3(\mathrm{CC}) \quad \mathrm{G} \\
4-2 \\
(36-39)\end{array}$ & $\begin{array}{l}6(C C) \\
7(C C) \\
(51-60)\end{array}$ & $\begin{array}{l}3-1 \\
3-2 \\
(14-16)\end{array}$ & $\mathbf{P}$ \\
\hline $\mathrm{T}$ & $\begin{array}{l}\text { Axoprunum } \\
\text { angelinum }\end{array}$ & $\mathrm{m}$ & $\begin{array}{l}2-2 \\
2-3 \\
(7-9)\end{array}$ & G & $\begin{array}{l}11-5 \\
11(\mathrm{CC}) \\
(96-100)\end{array}$ & M & & $\begin{array}{ll}5-1 & P \\
5-2 & P \\
(46-48) & \end{array}$ & $\begin{array}{l}8(C C) \\
9-1 \\
(69-71)\end{array}$ & $\begin{array}{l}4-3 \\
4-4 \\
(26-28)\end{array}$ & M \\
\hline & $\begin{array}{l}\text { Lamprocyrtis } \\
\text { neoheteroporos } \\
\rightarrow \text { L. haysi }\end{array}$ & & $\begin{array}{l}4-1 \\
4-3 \\
(25-29)\end{array}$ & G & $\begin{array}{l}17-6 \\
18-2 \\
(155-159)\end{array}$ & $\mathbf{P}$ & & & & & \\
\hline $\mathrm{T}$ & $\begin{array}{l}\text { Eucyrtidium } \\
\text { matuyamai }\end{array}$ & $\mathrm{e}$ & $\begin{array}{l}4(\mathrm{CC}) \\
5-1 \\
(34-35)\end{array}$ & $\mathbf{P}$ & & & & $\begin{array}{l}5-1 \\
5-2 \\
(46-48)\end{array}$ & $\begin{array}{l}14(\mathrm{CC}) \\
15-1 \\
(123-125)\end{array}$ & $\begin{array}{l}6-2 \\
6(C C) \\
(43-51)\end{array}$ & $P$ \\
\hline & $\begin{array}{l}\text { Lamprocyrtis } \\
\text { heteroporos } \\
\rightarrow \text { L. neoheteroporos }\end{array}$ & & $\begin{array}{l}6-1 \\
6-2 \\
(53-55)\end{array}$ & G & & & $\begin{array}{ll}4(\mathrm{CC}) \quad P \\
5-3 \\
(32-45)\end{array}$ & $\begin{array}{ll}* 5-5 & M \\
5-6 & M \\
(50-52) & \end{array}$ & $\begin{array}{l}* 22(\mathrm{CC}) \\
23-1 \\
(195-197)\end{array}$ & & \\
\hline B & $\begin{array}{l}\text { Eucyrtidium } \\
\text { matuyamai }\end{array}$ & m-e & $\begin{array}{l}9-1 \\
9-2 \\
(73-75) \\
\end{array}$ & G & & & & $\begin{array}{ll}5-5 & M \\
5-6 & M \\
(50-52) & \\
\end{array}$ & $\begin{array}{l}22(C C) \\
23-1 \\
(195-197)\end{array}$ & & \\
\hline & $\begin{array}{l}\text { Sphaeropyle } \\
\text { robusta } \\
\rightarrow S \text {. langii }\end{array}$ & & $\begin{array}{l}9-2 \\
9-3 \\
(75-77)\end{array}$ & $\mathrm{M}$ & & & & $\begin{array}{ll}5-2 & P \\
5-6 & P \\
(48-52) & \\
\end{array}$ & $\begin{array}{l}33-5 \\
34-4 \\
(321-330)\end{array}$ & & \\
\hline $\mathrm{T}$ & $\begin{array}{l}\text { Stichocorys } \\
\text { peregrina }\end{array}$ & $\mathrm{e}$ & $\begin{array}{l}14-1 \\
14-2 \\
(120-122)\end{array}$ & M & & & & & & & \\
\hline B & $\begin{array}{l}\text { Theocalyptra } \\
\text { davisiana }\end{array}$ & $\mathrm{m}$ & $\begin{array}{l}14-2 \\
14-3 \\
(122-124)\end{array}$ & M & & & & & & & \\
\hline & $\begin{array}{l}\text { Stylacontarium } \\
\text { aff. bispiculum } \\
\rightarrow \text { S. acquilonium }\end{array}$ & & $\begin{array}{l}14(\mathrm{CC}) \\
15-3 \\
(129-133)\end{array}$ & M & & & & & & & \\
\hline B & $\begin{array}{l}\text { Lamprocyrtis } \\
\text { heteroporos }\end{array}$ & $\mathrm{m}$ & $\begin{array}{l}15-2 \\
15-3 \\
(131-133)\end{array}$ & M & & & & & & & \\
\hline $\mathrm{T}$ & $\begin{array}{l}\text { Theocorys } \\
\text { redondoensis }\end{array}$ & $\mathrm{m}$ & $\begin{array}{l}15-2 \\
15-3 \\
(131-133)\end{array}$ & M & & & & & & & \\
\hline $\mathrm{T}$ & $\begin{array}{l}\text { Stichocorys } \\
\text { delmontensis }\end{array}$ & $\mathrm{m}$ & $\begin{array}{l}15-4 \\
15(\mathrm{CC}) \\
(135-139)\end{array}$ & M & & & & & $\begin{array}{ll}50-3 & P \\
52-2 & P \\
(632-689) & \end{array}$ & & \\
\hline
\end{tabular}


TABLE 9 - Continued

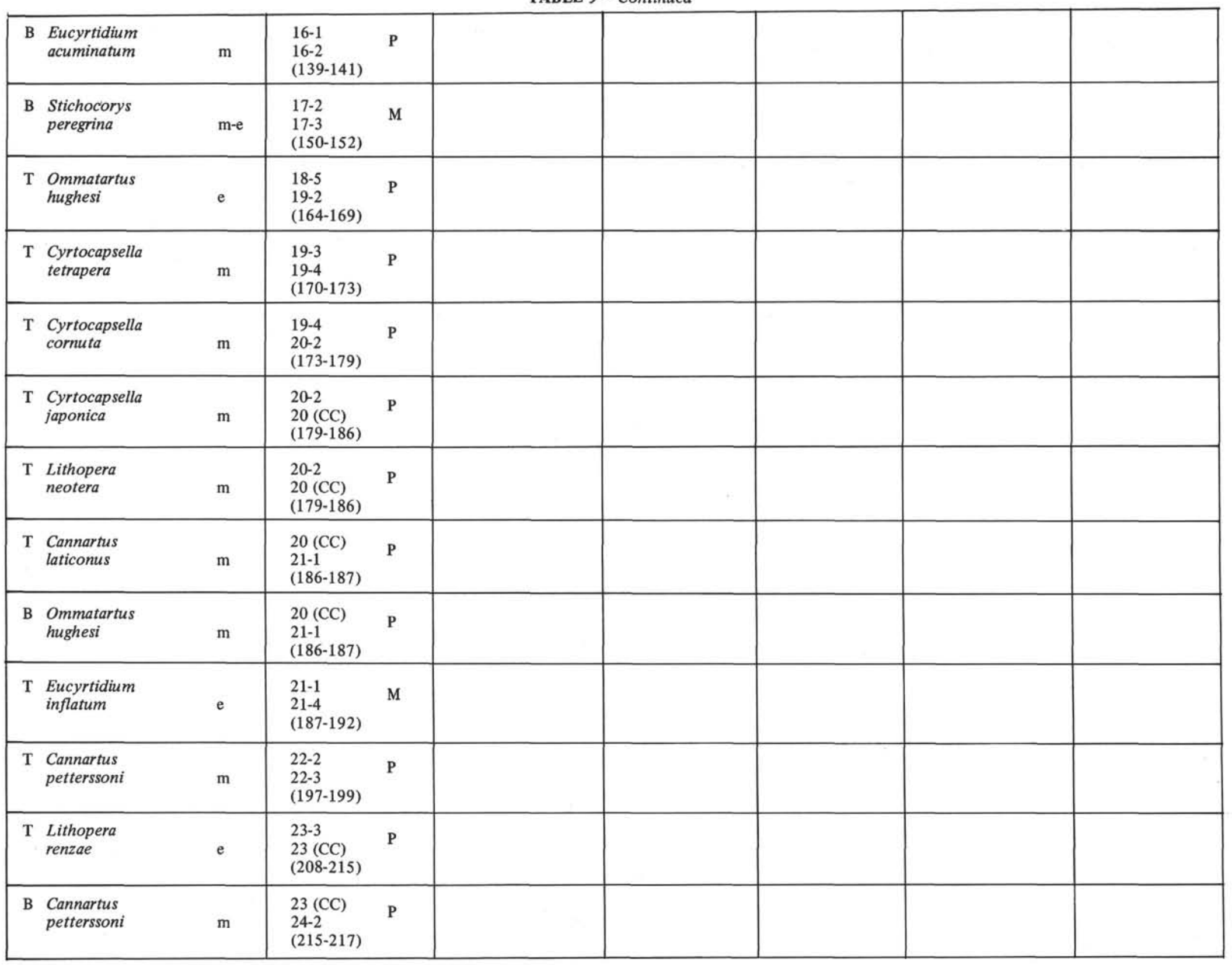


TABLE 9 - Continued

\begin{tabular}{|c|c|c|c|c|c|c|c|c|c|}
\hline \multicolumn{3}{|c|}{ Radiolarian Events } & \multicolumn{2}{|l|}{ Site 173} & \multirow[t]{2}{*}{ Site 175} & \multirow[t]{2}{*}{ Site 176} & \multirow[t]{2}{*}{ Site $177 \mathrm{~A}$} & Site 178 & \multirow[t]{2}{*}{ Site 179} \\
\hline B & $\begin{array}{l}\text { Stichocorys } \\
\text { delmontensis }\end{array}$ & $\mathrm{m}$ & $\begin{array}{l}27-2 \\
27-3 \\
(245-247)\end{array}$ & $\mathbf{P}$ & & & & $\begin{array}{ll}52-2 & P \\
54-1 & P \\
(689-744) & \end{array}$ & \\
\hline $\mathrm{T}$ & $\begin{array}{l}\text { Stichocorys } \\
\text { armata }\end{array}$ & $\mathrm{m}$ & $\begin{array}{l}27-3 \\
27-4 \\
(247-249)\end{array}$ & M & & & & & \\
\hline B & $\begin{array}{l}\text { Peripyramis } \\
\text { circumtexta }\end{array}$ & $\mathrm{m}$ & $\begin{array}{l}27-4 \\
27 \text { (CC) } \\
(249-253)\end{array}$ & M & & & & & \\
\hline B & $\begin{array}{l}\text { Eucyrtidium } \\
\text { inflatum }\end{array}$ & $\mathrm{m}-\mathrm{e}$ & $\begin{array}{l}27-4 \\
27(\mathrm{CC}) \\
(249-253)\end{array}$ & G & & & & & \\
\hline & $\begin{array}{l}\text { Cannartus } \\
\text { mammiferus } \\
\rightleftarrows \text { C. laticonus }\end{array}$ & & $\begin{array}{l}27-4 \\
28-1 \\
(249-254)\end{array}$ & P & & & & & \\
\hline B & $\begin{array}{l}\text { Lithopera } \\
\text { neotera }\end{array}$ & $\mathrm{m}-\mathrm{e}$ & $\begin{array}{l}28-2 \\
28-3 \\
(255-257)\end{array}$ & $P$ & & & & & \\
\hline B & $\begin{array}{l}\text { Theocorys } \\
\text { redondoensis }\end{array}$ & $\mathrm{m}$ & $\begin{array}{l}28-2 \\
28-3 \\
(255-257) \\
\end{array}$ & M & & & & & \\
\hline B & $\begin{array}{l}\text { Lithopera } \\
\text { renzae }\end{array}$ & $\mathrm{m}$ & $\begin{array}{l}29-2 \\
29 \text { (CC) } \\
(264-272)\end{array}$ & $\mathbf{P}$ & & & & & \\
\hline B & $\begin{array}{l}\text { Stichocorys } \\
\text { armata }\end{array}$ & $\mathrm{m}$ & $\begin{array}{l}29(\mathrm{CC}) \\
30(\mathrm{CC}) \\
(272-281)\end{array}$ & $\mathbf{P}$ & & & & & \\
\hline $\mathrm{T}$ & $\begin{array}{l}\text { Stichocorys } \\
\text { diploconus }\end{array}$ & $\mathrm{m}$ & $\begin{array}{l}29(\mathrm{CC}) \\
30(\mathrm{CC}) \\
(272-281)\end{array}$ & $\mathbf{P}$ & & & & & \\
\hline & $\begin{array}{l}\text { Cannartus } \\
\text { violina } \\
\rightarrow \text { C. mammiferus } \\
\end{array}$ & & $\begin{array}{l}30(\mathrm{CC}) \\
31-1 \\
(281-283) \\
\end{array}$ & $\mathbf{P}$ & & & & & \\
\hline $\mathrm{T}$ & $\begin{array}{l}\text { Dorcadospyris } \\
\text { simplex }\end{array}$ & $\mathrm{m}$ & $\begin{array}{l}30 \text { (CC) } \\
31-1 \\
(281-283)\end{array}$ & $P$ & & & & & \\
\hline B & $\begin{array}{l}\text { Stichocorys } \\
\text { diploconus }\end{array}$ & $\mathrm{m}$ & $\begin{array}{l}31-1 \\
31(\mathrm{CC}) \\
(283-286)\end{array}$ & $\mathbf{P}$ & & & & & \\
\hline
\end{tabular}

${ }^{\mathrm{a}} \mathrm{F}$ or explanation, see text (Radiolarian Events and Potential Datum Levels)

$\mathrm{b}_{\mathrm{T}}$ L. heteroporos $\mathrm{m}$ 
(1970), is well documented at Site 173. Here distinction of the two species is based on an abrupt change in size (Figure 2). Inflation of the second segment and coarsening of the latticework occurs considerably below this level in narrow specimens here included in $E$. calvertense; further study of this sequence may warrant separation of $E$. matuyamai at a somewhat lower level. No decendents of $E$. matuyamai are known after its extinction near the base of the Jaramillo paleomagnetic event at about 0.9 m.y.

4) Lamprocyrtis heteroposos - Lamprocyrtis haysi (Site 173). $L$. heteroporos first appears at approximately the Miocene-Pliocene boundary where it probably evolves from Lamprocyrtis(?) hannai (see Casey et al., 1972). In the lower Pleistocene Eucyrtidium matuyamai Zone, L. heteroporos evolves into $L$. neoheteroporos which, in the next younger Axoprunum angelinum Zone, evolves into $L$. haysi. The latter species is found in the youngest surface sediments and is presented to be still living.

\section{PRESERVATION}

Estimates of radiolarian preservation in this study are very imprecise and, with our meager knowledge of the factors affecting preservation of siliceous skeletons in the oceans and sediments, warrant only brief comments. Some incomplete specimens are observed in all samples, and it is often difficult to distinguish poor original preservation of this sort from breakage induced during prepraation. Preservation is considered "good" where a large number of species is present, and at least some skeletons of each species are reasonably complete. Samples in which incomplete specimens are the general rule are considered to have "poor" preservation. Samples with intermediate species diversity and specimen integrity are included in the "moderate" preservation category.

Selective solution of certain species is often evident and results, ultimately, in samples containing very few species which may themselves be quite well preserved and relatively abundant. Such samples, generally assigned a "moderate" preservation rating, are particularly evident in the Site 178 tabulations (Table 6).

Site 172 , with only a few radiolarians at the sediment surface, underlies the Central Water Mass where rates of organic productivity, as well as terriginous sediment supply, are low. Here radiolarians are generally scarce and poorly preserved. Along the Pacific Coast of North America, poor radiolarian recovery is noted particularly at Sites 174 and 176. Site 174 is characterized by high sedimentation rates on Astoria Fan, which results in dilution of skeletal remains by terriginous material. Site 176 lies on the continental shelf where radiolarians are generally scarce because of dilution and probably other factors. Poor preservation in the North Pacific sites (177-182) is characteristic of the region. Here, productivity is presumably high and terriginous dilution generally low; the relatively poor preservation of radiolarians (diatoms are usually abundant and wellpreserved) is not yet readily understood.

\section{PALEOECOLOGY}

The present state of knowledge of radiolarian ecology and the preliminary nature of this report preclude a detailed paleoecological discussion, but some general outlines of the ecologic significance of the results can be drawn.

The best trend can be seen at Site 173 (off Cape Mendocino, California). The Pliocene-Pleistocene assemblages here resemble those of the subarctic North Pacific, with essentially no representatives of the stratigraphically important species (e.g., members of the subfamily Artiscinae, Petrocanium praetextum, $P$. prismatium, Spongaster spp.) commonly encountered in equatorial regions. In the Miocene part of the sequence, however, many samples contain enough equatorial species (particularly the artiscins) to permit recognition of previously proposed equatorial biostratigraphic zones. This suggests a generally greater influence of equatorial waters during the middle and lower Miocene. The appearance of equatorial forms is variable, and it is likely that more detailed analysis could reveal fluctuations in tropical influence. These forms seem to appear in addition to, rather than as a replacement of, the normal California Current assemblage, suggesting that the tropical influence then, as now, took the form of incursions, probably subsurface, of equatorial water without an actual displacement of, or necessarily a wholesale change in, the prevailing oceanic climate.

Paleoecologic changes were not detected at other sites, all of whose faunas resemble those of the present overlying water masses. The only other comparably long sequence, at Site 178 , is poorly preserved in the pre-Pleistocene part. A few forms which occur in tropical regions (Stichocorys delmontensis, $S$. peregrina, Cyrtocapsella tetrapera, $C$. cornuta) also appear here, but these are probably more widely distributed forms that do not necessarily represent lower latitude influence.

\section{POSSIBLE MESOZOIC (FRANCISCAN?) OCCURRENCES}

At the bottom of Holes 173 and 174A, poorly preserved radiolarians (infilled and recrystallized) were encountered. At Site 173 a few specimens indicating a Mesozoic (probably Cretaceous) age (see Section 3 ) were observed. No age-diagnostic forms were identified at Site 174, but the overall aspects of both faunas were similar and quite unlike the clearly recognizable Tertiary assemblages.

This material resembles, in its preservation, radiolarians collected from cherts and related lithologies of the Franciscan "formation" on land. Thus it suggests, but does not prove, the existence of Franciscan rocks at or near the bottoms of these holes.

\section{SYSTEMATICS}

The classification followed here is based on recent revisions by Riedel $(1967,1971)$ and closely follows treatments by Riedel and Sanfilippo in previous volumes of this series $(1970,1971)$. Type specimens will be deposited in the U.S. National Museum, Washington, D. C.

In the synonomies, references following the original description are given in some cases to indicate a revised current concept of the species and/or a new generic combination. No attempt is made to give complete synonomies. Type species are cited only for genera not treated in recent literature. Species are arranged alphabetically with families and subfamilies. 


\section{Family COLLOSPHAERIDAE Muller 1858}

Members of this family are illustrated in the synchronopticon and identified to genus level employing the summary of genera presented by Riedel (1971).

\section{Family ACTINOMMIDAE Haeckel 1862, emend. Riedel 1967b} Genus AXOPRUNUM Haeckel

Axoprunum Haeckel, 1887 , p. 298. Type species (by monotypy) Axoprunum stauraxonium Haeckel (1887, p. 298, pl. 48, fig. 4).

Axoprunum angelinum (Campbell and Clark)

(Plate 1, Figures 13-16; Plate 6, Figures 14-18)

Stylosphaera angelina Campbell and Clark, 1944, p. 12, pl. 1, figs. 14-20.

Stylatractus universus Hays, 1970, p. 215, pl. 1, figs. 1, 2; Kling, 1971 , p. 1086 , pl. 1 , fig. 7.

Stylatractus sp. Hays, 1965 , p. 167, pl. 1, fig. 6 .

Remarks: These forms appear to be conspecific and closely related to the type species of the genus from which they differ principally by the presence of the short radial spines termed "primary spines" by Hays (1970). Forms with smooth cortical shell, probably attributable to the type species, are common in Neogene and Quaternary samples and may also be related to Paleogene forms included in Axoprunum pierinae Clark and Campbell (1941) by Riedel and Sanfilippo (in press).

\section{Genus SPHAEROPYLE Dreyer}

Sphaeropyle Dreyer, 1889 , p. 12. Type species (designated by Campbell, 1954), Sphaeropyle langii Dreyer.

\section{Sphaeropyle langii Dreyer}

(Plate 1, Figures 5-10; Plate 13, Figures 6-8)

Sphaeropyle langii Dryer, 1889 , p. 13, pl. 4, fig. 54; Hülsemann, 1963 , p. 17, fig. 9.

(?) Prunopyle antarctica Dreyer, 1889 , p. $24-25$, pl. 5, fig. 75 ; Riedel, 1958 , p. 225 , pl. 1, figs. 7,8 .

(?) Cromyechinus antarctica (Dreyer), Petrushevskaya, 1967, p. 2527 , figs. 13 , I-VII, and 14 , I-VII.

Remarks: It seems likely that all these forms are conspecific. Radial spines of the arctic and antarctic forms (as opposed to lower latitude forms) appear to be more strongly developed, and it is possible that they represent distinct subspecies.

Sphaeropyle robusta Kling, n. sp.

(Plate 1, Figures 11, 12; Plate 6, Figures 9-13; Plate 13, Figures 1-5)

Description: Four concentric shells. Innermost shell robust, spherical, with circular pores of uniform size, irregularly arranged, 3-4 per half-circumference. Second shell (outward) robust, spherical, with circular pores of fairly uniform size, quincuncially arranged, 6-7 per half-circumference, weakly framed. Third shell, usually relatively thin-walled (particularly in later representatives), spherical to subspherical, with circular pores of variable size but conspicuously large relative to other shells, 7-9 per half-circumference, with tiny by-spines at most bar intersections. Cortical shell robust, subspherical to elongate, protruding at one end, bearing a pylome which is surrounded with a corona of stout teeth; pores circular, irregularly arranged, usually with well-developed frames, about 20 per half-circumference; bar intersections often developed into short by-spines.

Approximately 6 radial bars connect the inner three shells, many times this number between cortical and next inner shell. Stout radial main-spines are usually short in Leg 18 material, but appear broken and could be better developed (preserved) elsewhere.

Earlier populations (Plate 6, Figures 11-13) are smaller with peristomal teeth fused into a rim. They are otherwise similar although it may eventually be possible to separate these forms as a new species.

Dimensions: Based on 10 specimens from $177 \mathrm{~A}-6-4(70-72 \mathrm{~cm})$ and 173-9-4 (85-87 cm): diameter of innermost shell 16-18 $\mu$, second shell $40-46 \mu$, third $100-110 \mu$, cortical (small diameter) $146-200 \mu$. Based on 10 specimens from 173-24-2 $(57-59 \mathrm{~cm})$ and 173-27-2 $(57-59 \mathrm{~cm})$ : innermost shell $10-20 \mu$, second $30-44 \mu$, third $62-100 \mu$, cortical $98-156 \mu$.
Remarks: This species differs from $S$. langii, its apparent descendent, primarily in its more robust cortical shell with framed pores. S. langii is generally smaller with a thin cortical shell bearing simple, cylindrical pores. Ancestry of this species is unknown.

Holotype: Plate 13 , Figures 4, 5; 173-16-1 $(70-72 \mathrm{~cm})$, slide 1 , $\mathrm{T} 35 / 4$.

Paratype: Plate 13, Figures 1-3; 177A-6-4 $(70-72 \mathrm{~cm})$, slide 1, T70/0.

\section{Genus STYLACONTARIUM Popofsky}

Stylacontarium Popofsky, 1912, p. 90. Type species (by monotypy) Stylacontarium bisipiculum Popofsky.

Stylacontarium sp. aff. S. bispiculum (Plate 6, Figures 19-23; Plate 14, Figures 5-8)

Stylacontarium bispiculum Popofsky, 1912, p. 91, pl. 2, fig 2. Axoprunum stauraxonium Haeckel, Casey et al., 1972, pl. 1, fig. 12.

Remarks: Specimens included here closely resemble the type species of the genus (Plate 15, Figures 11-14). They are found only in Miocene samples and apparently give rise to $S$. acquilonium (Hays) in the upper Miocene. They are retained in this tentative category pending further study of living material. Lighter shelled (Plate 14, Figures 7, 8) specimens, more closely resembling the figure of the type species, are probably conspecific with the commoner heavy-shelled specimens.

\section{Stylacontarium acquilonium (Hays)}

(Plate 1, Figures 17-20; Plate 14, Figures 1-4)

Druppatractus acquilonius Hays, 1970 , p. 214 , pl. 1, figs. 4, 5; Kling, 1971, p. 1086, pl. 1, figs. 5, 6.

Remarks: This species differs from $S$. bispiculum primarily by the elliptical shape of the outer medullary shell which protrudes at the connecting bars. This species also includes occasional lightershelled specimens (Plate 14, Figures 3, 4). It is transferred to the genus Stylacontarium because of close resemblance to the type species and its possible evolution from the type species or a closely related species.

\section{Subfamily ARTISCINAE Haeckel 1881, emend. Riedel 1967b}

Genus CANNARTUS Haeckel 1881, emend, Riedel 1971

\section{Cannartus laticonus Riedel}

(Plate 7, Figure 7)

Cannartus laticonus Riedel, 1959, p. 291, pl. 1, fig. 4.

Cannartus mammiferus (Haeckel) (Plate 7, Figure 9)

Cannartidium mammiferum Haeckel, 1887 , p. 375 , pl. 39 , fig. 16 . Cannartus mammiferus (Haeckel), Riedel, 1959, p. 291, pl. 1, fig. 4.

\section{Cannartus (?) petterssoni Riedel and Sanfilippo} (Plate 7, Figure 8)

Cannartus (?) pettersonni Riedel and Sanfilippo, 1970, p. 520-521, pl. 14 , fig. 3 .

\section{Cannartus violina Haeckel} (Plate 7, Figure 10)

Cannartus violina Haeckel, 1887, p. 538, pl. 39, fig. 10; Riedel, 1959 , p. 290, pl. 1, fig. 3.

Genus OMMATARTUS Haeckel 1881, emend. Riedel 1971

Ommatartus hughesi (Campbell and Clark) (Plate 7, Figure 6)

Ommatocampe hughesi Campbell and Clark, 1944, p. 23, pl. 3, fig. 12.

Ommatartus hughesi (Campbell and Clark), Riedel and Sanfilippo, 1970 , p. 521.

\section{Subfamily SATURNALINAE Deflandre 1953}

Genus SATURNALIS Haeckel 1881, emend. Nigrini 1967

Saturnalis Haeckel 1881, p. 450; Nigrini, 1967, p. 24. Type species (indicated by Campbell, 1954) Saturnalis circularis Haeckel (1887, p. 131). 
Saturnalis circularis Haeckel

(Plate 1, Figures 21-25; Plate 7, Figures 1-5)

Saturnalis circularis Haeckel, 1887, p. 131; Nigrini, 1967, p. 25, pl. 1 , fig. 9.

Saturnalis planetes Haeckel, 1887, p. 142, pl. 16, fig. 7; Hays, 1965 , p. 167 , pl. 1 , fig. 5 .

Remarks: Nigrini (1967) synonomizes several additional species with $S$. circularis, concluding that there is only one living species. This same species appears to range back through the Miocene in Leg 18 material.

Family SPONGODISCIDAE Haeckel 1862, emend. Riedel 1967b Genus SPONGOCORE Haeckel

Spongocore Haeckel, 1887 , p. 345 . Type species (designated by Campbell, 1954) Spongocore velata Haeckel (1887, p. 346)

\section{Spongocore puella Haeckel}

(Plate 7, Figures 18-22)

Spongocore puella Haeckel, 1887, p. 347 , pl. 48, fig. 6; Benson, 1964 , pl. 1 , fig. 21 ; Nigrini, 1970 , p. 168 , pl. 2 , fig. 3 .

Spongocore lata Campbell and Clark, 1944, p. 22, pl. 3, figs. 5, 6. Spongocore puer Campbell and Clark, 1944, p. 22, pl. 3, figs. 7-9.

Remarks: Relationship of this species to the type species of the genus is uncertain, but the combination is retained for convenience. It appears the normal variability within the species could include the forms separated by Campbell and Clark.

\section{Genus SPONGOTROCHUS Haeckel}

Spongotrochus Haeckel, 1860 , p. 844 . Type species (indicated by Campbell, 1954), Spongotrochus brevispinus Haeckel (1861, p. $844 ; 1862$, pl. 27 , figs. 4,5 ).

\section{Spongotrochus (?) glacialis Popofsky}

(Plate 2, Figures 4-6)

Spongotrochus glacialis Popofsky, 1908, p. 228-229, pl. 26, fig. 8; pl. 27, fig. $1 ;$ pl. 28 , fig. 2.

Spongotrochus ? glacialis Popofsky, Riedel 1958, p. 227-228, pl. 2, figs. 1,2 ; text-fig. 1 .

Remarks: Relationship of this species to the type species of the genus is still uncertain. Strict adherence to the species description as emended by Riedel (1958) is necessary to distinguish this species from similar, but unrelated spongodiscids with peripheral spines.

\section{Genus XIPHOSPIRA Haeckel}

Remarks: This genus is used with the broad definition of Sanfilippo and Riedel (in press), allowing forms with concentric, spiral, or irregular rings with or without radial spines.

Xiphospira sp. cf. X. circularis (Clark and Campbell) (Plate 2, Figures 1-3; Plate 7, Figures 11-17)

Porodiscus circularis Clark and Campbell, 1942, p. 42, pl. 2, figs. 2, 6,10 .

Xiphospira circularis (Clark and Campbell) Sanfilippo and Riedel (in press).

(?) Stylodictya heliozoides Campbell and Clark, 1944, p. 25, pl. 3, fig. 17.

(?) Stylodictya camerina Campbell and Clark, 1944, p. 26, pl. 3, figs. 18, 19, 21.

(?) Stylodictya ornata Campbell and Clark, 1944, p. 26, pl. 3, fig. 20.

(?) Stylodictya cornuspira Campbell and Clark, 1944, p. 27, pl. 3, fig. 22.

Remarks: According to the broad concept of this species employed by Sanfilippo and Riedel (in press), these forms are conspecific with the ones they describe, and with those illustrated by Campbell and Clark, although some of the specimens included here possess a greater number of rings and the inner ones are less consistently elliptical.

\section{Family LITHELIIDAE Haeckel 1862}

Genus SPIREMA Haeckel

Spirema Haeckel, 1881 , p. 464. Type species (indicated by Campbell, 1954), Spirema lentellipsis Haeckel, 1887, p. 692.
Spirema sp.

(Plate 7, Figures 23-25)

Remarks: Forms included here have a simple, evolute, planispirally coiled cortical shell with a smooth outer wall lacking radial spines. An understanding of their relationship to other species such as Lithelius nautiloides Popofsky, 1908, requires further study. They are tentatively included in the genus Spirema to distinguish them from spiny litheliids. They are apparently restricted to the Miocene at Site 173 .

Family ACANTHODESMIIDAE Haeckel 1862, emend. Riedel 1967b

Remarks: Members of this family are illustrated on Plates 2 and 8. Some figures are tentatively identified, but because of the uncertainty of the relationships of most of the forms, only one species is treated in the systematic section.

\section{Genus DORCADOSPYRIS Haeckel 1881 \\ Dorcadospyris simplex (Riedel) (Plate 8, Figure 27)}

Brachiospyris simplex Riedel, 1959, p. 293, pl. 1, fig. 10.

Dorcadospyris simplex (Riedel) Riedel and Sanfilippo, 1970, p. 523, pl. 15 , fig. 6 .

Family THEOPERIDAE Haeckel 1881, emend. Riedel 1967b Genus BATHROPYRAMIS Haeckel

Bathropyramis Haeckel, 1882 , p. 428 . Type species (indicated by Campbell, 1954), Bathropyramis acephala Haeckel (1887, p. 1159)

Bathropyramis woodringi Campbell and Clark (Plate 2, Figures 20-23; Plate 9, Figures 5, 7, ? Figures 4, 6)

Bathropyramis woodringi Campbell and Clark, 1944, p. 39 , pl. 5 , figs. $21,22$.

Peripyramis circumtexta Haeckel, Casey, 1971, pl. 23.1, fig. 11, Casey et al., 1972, pl. 2, fig. 4 .

\section{Genus CLATHROCYCLAS Haecke}

Clathrocyclas Haeckel, 1882, p. 434. Type species (indicated by Campbell, 1954), Clathrocyclas principessa Haeckel (1887, p. 1386, pl. 74, fig. 7).

\section{Clathrocyclas cabrilloensis Campbell and Clark}

(Plate 9, Figures 23-25)

Clathrocyclas cabrilloensis Campbell and Clark, 1944, p. 48 , pl. 7 , figs. 1-3.

Remarks: Some forms figured on the synchronopticon, and apparently restricted to the Miocene, are identified as this species. Its retention in this genus and relation to other forms included in Clathrocyclas are uncertain.

\section{Clathrocyclas spp.}

(Plate 3, Figures 17-22; Plate 9, Figures 26-31)

Remarks: Included in this group, which needs revision, are a number of both open and closed forms related to Clathrocyclas bicornia Hays (1965) and to Theoclayptra bicornis (Popofsky) Riedel (1958). These are generally smaller forms with cylindrical terminal segments which may bear a narrow terminal rim. They are distinguished, thus, from broadly flaring (although probably related) forms discussed under the genus Theoclayptra. No relationship to the type species of the genus is assumed, which is used as a temporary convenience.

\section{Genus CORNUTELLA Ehrenberg}

Cornutella Ehrenberg, 1838 , p. 128. Type species (indicated by Campbell, 1954), Cornutella clathrata Ehrenberg (1838, p. 129; 1844 , p. 77,1856 , pl. 22 , fig. 39 ).

Cornutella profunda Ehrenberg

(Plate 3, Figures 1-4; Plate 9, Figures 8-17)

Cornutella clathrata $\beta$ profunda Ehrenberg, 1956, pl. 35B, fig. 21; Bailey, 1856, p. 2, pl. 1, fig. 23. 
Cornutella profunda Ehrenberg, 1858, p. 31; Riedel, 1958, p. 232, pl. 3 , figs. 1,2 ; Nigrini, 1967 , p. 60 , pl. 6 , figs. 5 a-5c.

Cornutella paloverdensis Campbell and Clark, 1944, p. 40, pl. 5, figs. 17, 20, 23, 24, 25.

Remarks: This species includes numerous intergrading variants, many of which have been described as separate species, and which are included in a lengthy synonomy by Nigrini (1967).

\section{Genus CYRTOCAPSELLA Haeckel 1887 \\ Cyrtocapsella cornuta Haeckel \\ (Plate 11, Figures 16-18)}

Cyrtocapsa (Cyrtocapsella) cornuta Haeckel, 1887, p. 1513, pl. 78, fig. 9.

Cyrtocapsella cornuta Haeckel, Sanfilippo and Riedel, 1970, p. 453, pl. 1, figs. 19-20.

\section{Cyrtocapsella japonica (Nakaseko)}

(Plate 11, Figures 19, 20)

Eusyringium japonicum Nakeseko, 1963, p. 193, pl. 4, figs. 1-3.

Cyrtocapsella japonica (Nakaseko) Sanfilippo and Riedel, 1970, p. 452 , pl. 1, figs. 13-15.

\section{Cyrtocapsella tetrapera Haeckel}

(Plate 11, Figures 12-15)

Cyrtocapsa (Cyrtocapsella) tetrapera Haeckel, 1887, p. 1512, pl. 78, fig. 5 .

Cyrtocapsella tetrapera Haeckel, Riedel and Sanfilippo, 1970, p. 453, pl. 1, figs. 16-18.

\section{Genus DICTYOPHIMUS Ehrenberg}

Dictyophimus Ehrenberg, 1847, p. 54. Type species (by monotypy), Dictyophimus crisiae.

Nigrini (1967, p. 68) discusses the correct type species for this genus.

\section{Dictyophimus crisiae Ehrenberg}

(Plate 4, Figures 11-15; Plate 10, Figures 18-20)

Dictyophimus crisiae Ehrenberg, 1854, p. 241, Nigrini, 1967, p. 66, pl. 6 , figs. $7 \mathrm{a}, 7 \mathrm{~b}$.

Pterocorys hirundo Haeckel, Casey, 1971, pl. 23.1, figs. 6, 7; Petrushevskaya, 1967 (partim), p. 115, figs. 4, 5 .

(?) Petrocorys hirundo Haeckel, Riedel, 1958, p. 238, pl. 3, fig. 11, pl. 4, fig. 1; Petrushevskaya, 1967 (partim), p. 115, figs. 1-3. Remarks: The smaller forms with spiny cephalis and less developed abdomen, figured by Riedel (1958) and Petrushevskaya (1967), may well represent a distinct species.

\section{Genus EUCYRTIDIUM Ehrenberg 1847 \\ Eucyrtidium acuminatum (Ehrenberg) (Plate 4, Figures 20-23)}

Lithocampe acuminatum Ehrenberg, 1844, p. 84.

Eucyrtidium acuminatum (Ehrenberg), 1847, p. 43; Nigrini, 1967, p. 81 , pl. 8 , figs. 3 a, 3 b.

\section{Eucyrtidium calvertense Martin}

(Plate 4, Figures 16, 18, 19; Plate 11, Figures 1-5)

Eucyrtidium calvertense Martin, 1904, p. 450, pl. 130, fig. 5; Hays, 1965 , p. 181 , pl. 3 , fig. $4 ; 1970$, p. 213 , pl. 1 , fig. 6 .

\section{Eucyrtidium inflatum Kling n. sp.}

(Plate 11, Figure 7,; Figure 8; Plate 15, Figures 7-10)

Description: Cephalis subspherical to hemispherical, with sparce circular pores; bears a tiny apical spine which is inconspicuous or lacking in many specimens. Collar stricture indistinct. Thorax slightly inflated conical, moderate rough-surfaced, with longitudinal ribs on lower part separating longitudinal rows of circular pores. Lumbar stricture distinct. Abdomen and one or more postabdominal segments define characteristic inflated, truncate fusiform section of the test without externally expressed strictures. Pores in this section are circular, arranged in strict longitudinal rows separated by distinct, sharp-crested ridges. Widest part of shell in abdomen or spanning division between abdomen and fourth segment. In some specimens, gentle stricture separates symmetrical fusiform section from fragmentary lower chamber.

Dimensions: Based on 15 specimens from 173-25-5 $(50-52 \mathrm{~cm})$ and 173-22-2 $(57-59 \mathrm{~cm})$, maximum width $80-94 \mu$, length of cephalis to distal end of thorax $36-44 \mu$, to end of abdomen, $90-100 \mu$, to end of fourth segment $120-136 \mu$; abdominal pores, minimum $2-4 \mu$, maximum $5-9 \mu$, average close to maximum.

Remarks: This species differs from $E$. calvertense in the inflated fusiform section of the shell and relatively large and widely spaced pores. $E$. calvertense is seldom widest at the abdomen, as $E$. inflatum nearly always is. $E$. matuyamai is larger and inflated at more distal segments.

Holotype: Plate 15, Figures 7, 8; 173-22-2 (57-59), slide 1, $\mathrm{T} 45 / 3$.

\section{Eucyrtidium matuyamai Hays}

(Plate 4, Figure 17)

Eucyrtidium matuyamai Hays, 1970, p. 213, pl. 1, figs. 7-9; Kling, 1971 , p. 1088 , pl. 1 , fig. 4 .

Remarks: It is somewhat more difficult to distinguish the first appearance of this species from $E$. calvertense at Site 173. E. calvertense is somewhat heavier-shelled with coarser longitudinal furrows in the upper Pliocene of this site. The distinction has been made on the basis of an arbitrary visual estimate of a trend toward larger size based on histogram plots of maximum width (Figure 2), While the absolute values are not considered significant, deviation from a stable average size can be seen. It may also be possible to distinguish $E$. matuyamai on the basis of a markedly inflated thorax (Hays, 1972, oral communication), in which case its first appearance might be placed one or two cores lower in the section.

\section{Genus LIPMANELLA Loeblich and Tappan 1961}

Lipmanella Loeblich and Tappan, 1961, p. 229 (= Dictyoceras Haeckel 1862). Type species, Lithornithium dictyoceras Haeckel (=Dictyoceras virchowii Haeckel).

Lipmanella dictyoceras (Haeckel)

(Plate 4, Figures 24-26)

Lithornithium dictyoceras Haeckel, 1860 , p. 840.

Dictyoceras virchowii Haeckel, 1862, p. 333, pl. 8, figs. 1-5.

Lipmanella virchowii (Haeckel) Petrushevskaya, 1971, p. 220, fig. 198.

Remarks: Dictyoceras virchowii is an invalid name proposed arbitrarily by Haeckel in 1862 for Lithornithium dictyoceras.

\section{Genus LITHOPERA Ehrenberg 1847}

Lithopera bacca Ehrenberg

(Plate 11, Figures 21, 22)

Lithopera bacca Ehrenberg, 1872a, p. 314; 1872b, pl. 8, fig. 1; Sanfilippo and Riedel, 1970, p. 455, pl. 1, fig. 29.

\section{Lithopera neotera Sanfilippo and Riedel} (Plate 11, Figure 23)

Lithopera neotera Sanfilippo and Riedel, 1970, p. 454, pl. 1, figs. 24-26, 28.

\section{Lithopera renzae Sanfilippo and Riedel} (Plate 11, Figure 25)

Lithopera renzae Sanfilippo and Riedel, 1970, p. 454, pl. 1, figs. 21-23, 27.

\section{Lithopera thornburgi Sanfilippo and Riedel} (Plate 11, Figure 24)

Lithopera thornburgi Sanfilippo and Riedel, 1970, p. 455, pl. 2, figs. 4-6.

\section{Genus LYCHNOCANOMA Haeckel}

Lychnocanoma Haeckel, 1887 , p. 1229. Type species (designated by Campbell, 1954). Lychnocanoma clavigerum Haeckel (1887, p. 1230 , pl. 61, fig. 4). 


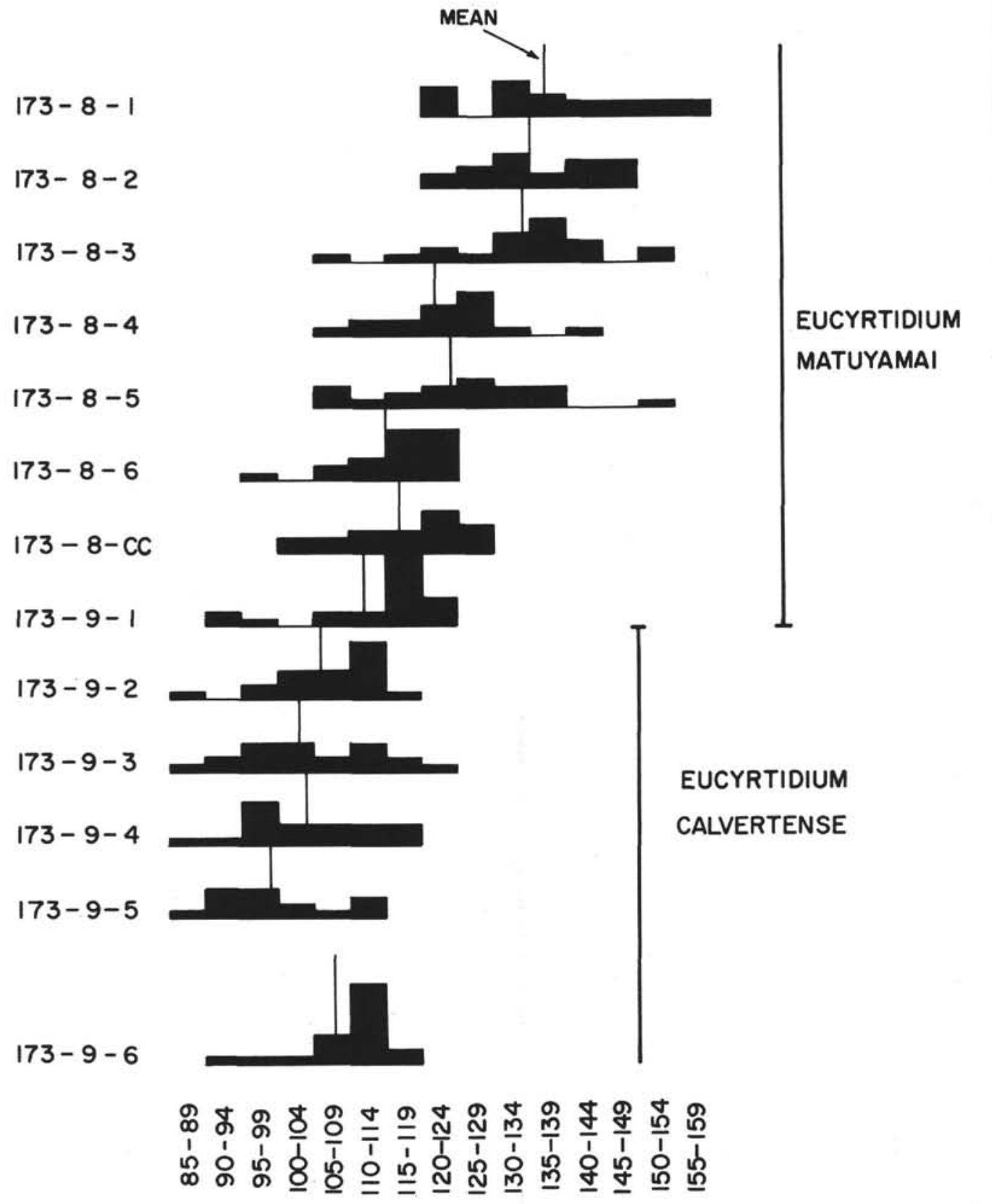

Figure 2. Histograms of maximum width $(\mu)$ of Eucyrtidium calvertense and Eucyrtidium matuyamai.

Lychnocanoma grande (Campbell and Clark) (Plate 10, Figures 10-14)

Lychnocanium grande Campbell and Clark, 1944, p. 42, pl. 6, figs. 3-6.

Remarks: Miocene specimens are referred to this species with confidence, but the relationship of similar younger forms to this species and to forms normally referred to the genus Pterocanium needs further study.

\section{Genus PERIPYRAMIS Haeckel}

Peripyramis Haeckel, 1881, p. 428. Type species (indicated by Campbell, 1954), Peripyramis circumtexta Haeckel.

Peripyramis circumtexta Haeckel

(Plate 2, Figures 15-16; Plate 9, Figures 1-3)

Peripyramis circumtexta Haeckel, 1887, p. 1162, pl. 54, fig. 5; Riedel, 1958, p. 231, pl. 2, figs. 8, 9. 


\section{Genus PTEROCANIUM Ehrenberg 1847}

Remarks: Forms normally assigned to this genus show considerable variability and possible intergradation in Leg 18 material. Pending futher study, two species are tentatively identified in the plates.

\section{Pterocanium korotnevi (Dogiel)}

(Plate 4, Figures 1-4; ? Plate 10, Figures 6-9)

Pterocorys korotnevi Dogiel, Dogiel and Reshetnyak, 1952, p. 17, fig. 11.

Pterocanium korotnevi (Dogiel), Nigrini, 1970, p. 170, pl. 3, figs. $10,11$.

\section{Pterocanium trilobum (Haeckel)} (Plate 4, Figures 5-8)

Dictyopodium trilobum Haeckel, 1860 , p. $839 ; 1862$, p. 340 , pl. 8 , figs. 6-10.

Pterocanium trilobum (Haeckel), 1887, p. 1333; Hays, 1965, p. 177, pl. 3, fig. 10; Nigrini, 1967, p. 71, pl. 7, figs. 3a, 3b.

Genus STICHOPERA Haeckel 1881

Stichopera pectinata Haeckel group

(Plate 3, Figures 25-27; Plate 10, Figures 1-5)

Stichopera pectinata Haeckel, 1887, p. 1449, pl. 75, fig. 11.

Cyrtopera laguncula Haeckel, 1887, p. 1451, pl. 75, fig. 10.

Remarks: Included temporarily in this group are forms with numerous segments increasing regularly in size distally and terminating in a bulbous closed chamber. Although the terminal segment is often not preserved, all the illustrated forms appear to be closely related. Probably related to this group are two illustrations (Plate 3, Figures 23, 24) of a form identified as Stichophormis novena Haeckel (1887, p. 1455, pl. 79, fig. 9). Assignment to this genus is uncertain, but the group is unrelated to the type species of Cyrtopera (Cyrtopera thoracoptera Haeckel, 1887, p. 1450, pl. 75, fig. 3 ; indicated by Campbell, 1954).

\section{Genus STICHOCORYS Haeckel 1881 \\ Stichocorys armata (Haeckel) \\ (Plate 13, Figure 11)}

Cytophormis armata Haeckel, 1887, pl 1460, pl. 78, fig. 17.

Stichocorys armata (Haeckel), Riedel and Sanfilippo, 1971, p. 1595, pl. 2E, figs. 13-15.

\section{Stichocorys delmontensis (Campbell and Clark)}

$$
\text { (Plate 11, Figures 8-10) }
$$

Eucyrtidium delmontense Campbell and Clark, 1944, p. 56, pl. 7, figs. 19, 20.

Stichocorys delmontensis (Campbell and Clark), Sanfilippo and Riedel, 1970, p. 451, pl. 1, fig. 9.

Stichocorys diploconus (Haeckel)

(Plate 11, Figure 11; Plate 13, Figure 12)

Cyrtocapsa diploconus Haeckel, 1887, p. 1513, pl. 78, fig. 6 .

Stichocorys diploconus (Haeckel) Sanfilippo and Riedel, 1970, p. 451 , pl. 1 , figs. 31,32 .

\section{Stichocorys peregrina (Riedel)}

(Plate 4, Figure 27; Plate 11, Figure 29; Plate 13; Figures 9, 10)

Eucyrtidium elongatum peregrinum Riedel, 1953, p. 812, pl. 85, fig. 2 .

Stichocorys peregrina (Riedel) Sanfilippo and Riedel, 1970, p. 451, pl. 1 , fig. 10.

Remarks: Specimens from Site 173 differ from those normally encountered in equatorial regions in having a less markedly conical third segment, and it is hardly conical at all in others. However, the fourth segment is still as wide as the third and thicker-walled than the fifth and subsequent segments. Thus, specimens with four, as opposed to three, differentiated initial segments permit distinction of this species from Stichocorys delmontensis. The forms discussed here resemble ones from the Mediterranean region attributed to $S$. peregrina (Riedel and Sanfilippo, 1970, pl. 8, fig. 5; Sanfilippo et al., in press). It may be proper to segregate some of these variants as subspecies.

\section{Genus THEOCALYPTRA Haeckel}

Theocalyptra Haeckel, 1881 , p. 434. Type species (indicated by Campbell, 1954) Theocalyptra veneris Haeckel (1887, p. 1397). Remarks: In addition to the following species, a number of similar (though not necessarily related) open forms are tentatively placed in this genus in captions of the synchronopticon.

\section{Theocalyptra davisiana (Ehrenberg)}

(Plate 3, Figures 9-12, 28; ? Plate 3, Figures 5-8)

Cycladophora ? davisiana Ehrenberg, 1861, p. 297; 1873, pl. 2, fig. 11; Petrushevskaya, 1967 , p. 122 , figs. $69,70$.

Theocalyptra davisiana (Ehrenberg) Riedel, 1958, p. 239, pl. 4, figs. 2, 3, text-fig. 10 .

Remarks: Previous authors seem to have included forms with smoothly conical (though asymmetrical) shapes (Plate 3, Figures 5-8) as well as forms with sharply inflected outlines (Plate 3, Figures 9-12). The latter appear to be no older than Pliocene, while the former may range back into the Miocene (and possibly earlier). These should possibly be described as separate species.

\section{Genus THEOCORYS Haeckel 1881}

Remarks: The following species is transferred to this genus as a temporary convenience until its relationships with other forms are better understood.

\section{Theocorys redondoensis (Campbell and Clark)} (Plate 11, Figures 26-28)

Theocyrtis redondoensis Campbell and Clark, 1944, p. 49, pl. 7, fig. 4 ; Casey et al., 1972, pl. 2, fig. 3.

Remarks: The cephalic structure of this species necessitates its transfer to a theoperid genus. The type species of Theocyrtis (Eucyrtidium barbadense Ehrenberg, 1873, p. 226, 1875, pl. 9, fig. 7) belongs to the family Pterocoryidae.

\section{Family CARPOCANIIDAE Haeckel 1881, emend. Riedel 1967b}

Remarks: Members of this family are illustrated but not further identified.

\section{Family PTEROCORYIDAE Haeckel 1881, emend. Riedel 1967b} Genus LAMPROCYRTIS Kling, n. gen.

Type species: Lamprocyclas heteroporos Hays, 1965, p. 179, pl. 3 , fig. 1 .

Definition: This genus is erected to accommodate the lineage from Lamprocyrtis heteroporos through $L$. haysi and possibly $L$. (?) hannai, a possible ancestor of the lineage. It is difficult to characterize because of rather marked differences between the initial and latest members of the lineage. Included are two- (later) and three- (earlier) segmented forms with a usually open, indistinctly three-lobed cephalis which tends to be cylindrical. The cephalis bears one stout, three-bladed apical horn and commonly one or more accessory spines. Pores of the post-cephalic segment(s) usually increase in size distally, earlier species displaying a distinctive discontinuity in pore size. The shells terminate in a single, irregular row of axially to inwardly directed teeth which sometimes occupy a weakly developed peristome. Teeth occasionally occur outside the terminal row, but do not constitute a distinct second row as in Lamprocyclas Haeckel, 1882.

Lamprocyrtis (?) hannai (Campbell and Clark)

(Plate 5, Figures 12-14; Plate 12, Figures 10-14)

Calocyclas hannai Campbell and Clark, 1944, p. 48, pl. 69, figs. 21, 22; Casey et al., 1972, pl. 2, fig. 19.

(?) Calocyclas margatensis Campbell and Clark, 1944 , p. 47, pl. 6, figs. 17, 18; Casey et al., 1972, pl. 3, figs. 10-22.

Remarks: This three-segmented species with a single row of terminal teeth seems a likely ancestor of $L$. heteroporos. It is assigned tentatively to this genus pending more detailed comparisons. The differences between Campbell and Clark's two species are probably gradational. 
Lamprocyrtis haysi Kling, n. sp.

(Plate 5, Figures 15, 16; Plate 15, Figures 1-3)

Conarachnium ? sp. Nigrini, 1968 (partim), p. 56, pl. 1, fig. 5a.

(?) Conarachnium ? sp. Nigrini, 1968 (partim), p. 56, pl. 1, fig.5b.

Description: Two-segmented forms with hemispherical to cylindrical cephalis, commonly open, with irregularly arranged circular to elliptical pores and a three-bladed apical spine and usually one or more accessory spines. Collar stricture indistinct. Thorax inflated conical, thin-walled, smooth, with subcircular (rarely elliptical to irregular) pores generally aligned in longitudinal and transverse rows (rarely irregularly arranged) and gradually increasing in size distally. Thorax terminates, sometimes slightly constricted, with an irregular row of small teeth; a weak peristome is seldom developed.

Dimensions: Based on 15 specimens from 173-4-3 (57-59) and 173-3-3 (106-108), maximum width $80-90 \mu$ length from top of cephalis $100-150 \mu$, thoracic pores $2-20 \mu$.

Remarks: Lamprocyrtis haysi is distinguished from $L$. neoheteroporos by the lack of a sharp horizontal discontinuity in the size of thoracic pores. Although the transition is gradual, recognition of the $L$. haysi morphotype is based on the lack of a distinct circumferential discontinuity across which pores approximately double in size. In $L$. haysi pores tend to be aligned vertically over the length of the shell whereas in $L$. neoheteroporos one distal row of pores tends to occupy the space of 2 proximal rows. Specimens of $L$. haysi, with irregularly arranged pores including some exceptionally large ones, resemble $L$. neoheteroporos, but again lack the consistent horizontal discontinuity. Nigrini's (1968) description and figure $5 \mathrm{a}$ agree well with this species, but figure $5 \mathrm{~b}$ appears to be longer and more broadly flared than the Leg 18 specimens. The species is named for James D. Hays who first pointed out the stratigraphic potential of this group.

Holotype: Plate 15, Figure 1, 173-2-2 (45-57), slide 1, S29/2.

Lamprocyrtis heteroporos (Hays)

(Plate 5, Figures 19-21; Plate 15, Figure 6)

Lamprocyclas heteroporos Hays, 1965, p. 179, pl. 3, fig. 1; Kling, 1971 , p. 1088 , pl. 1, fig. 1 .

Lamprocyclas heteroporos ? Hays, 1970, p. 214, pl. 1, fig. 3.

Lamprocyrtis neoheteroporos Kling, $\mathrm{n}$. sp.

(Plate 5, Figures 17, 18; Plate 15, Figures 4, 5)

Description: Two segmented forms with cephalis hemispherical to cylindrical, commonly open; with a stout three-bladed apical horn and usually one or more accessory spines; with circular to elliptical, irregularly arranged pores. Collar stricture indistinct. Thorax proximally conical, distally cylindrical to slightly inflated; circular to elliptical or irregular pores, longitudinally aligned, increasing in size distally, becoming two or more times as large along a horizontal discontinuity as in $L$. heteroporos. Smaller pores may again appear near mouth. Although early specimens show a slight, gentle indentation of the wall at this discontinuity, there is no internal stricture or septal ring separating two segments. Mouth, sometimes slightly constricted, surrounded by irregular row of small teeth; a weak peristome is seldom developed. Later specimens become smaller and more inflated.

Dimensions: Based on 15 specimens from 173-6-1(108-110) and $173-4$ (CC), maximum width $74-90 \mu$, length from top of cephalis $100-130 \mu$, proximal pore diameter $2-14 \mu$, (average near upper value), distal pore diameter 6-28 $\mu$ (average near upper value).

Remarks: This species strongly resembles $L$. heteorporos, but lacks the distinct lumbar stricture of that species. The transition is gradual, the first appearance of the $L$. neoheteroporos morphotype defined when the last traces of an internal septal ring at the lumbar stricture disappear. The transition to $L$. haysi is discussed under that species.

Holotype: Plate 15, Figure 5, 173-5-3(57-59), slide 1, X34/2.

\section{Genus SETHOCORYS Haeckel}

Sethocorys Haeckel, 1881, p. 430. Type species (indicated by Campbell, 1954), Sethocorys achillis Haeckel (1887, p. 130, pl. 62 , fig. 8).
Sethocorys spp.

(Plate 12, Figures 15-18)

Remarks: Included in this group, illustrated but not tabulated, are forms apparently related to the type species of Sethocorys. Some specimens, in addition to a smooth peristome, bear weakly developed terminal teeth. Raised longitudinal ridges separate rows of pores on the thorax of some specimens.

\section{Genus THEOCONUS Haeckel}

Theoconus Haeckel, 1887 , p. 1399. Type species (designated by Campbell, 1954) Eucyrtidium zancleum Müller.

Theoconus zancleus (Müller) (?)

(Plate 5, Figures 9-11; ? Plate 12, Figures 7-9)

Eucyrtidium zancleum Müller, 1855 , p. $672 ; 1858$, pl. 6, figs. 1-3. Theoconus zancleus (Müller) Haeckel, 1887, p. 1399.

Remarks: Forms included here have a less expanded abdomen and, consequently, narrower terminal opening than the earliest concepts of $T$. zancleus. Miocene specimens, questionably referred to this species, are smaller with abdomens approaching cylindrical shapes with occasional peristomes and longitudinal ridges separating rows of pores; these could perhaps be separated as a new species.

\section{Family ARTOSROBIIDAE Riedel 1967a}

Remarks: Members of this family are treated in a manner similar to that of Riedel and Sanfilippo (1971).

\section{Genus ARTOSTROBIUM Haeckel 1887}

Artostrobium auritum (Ehrenberg) group (Plate 5, Figures 27-30; Plate 12, Figures 24-27)

Lithocampe aurita Ehrenberg, 1844, p. 84; 1854, pl. 22, fig. 25. Artostrobium auritum (Ehrenberg) group, Riedel and Sanfilippo, 1971 , p. 1599 , pl. $1 \mathrm{H}$, figs. $5-8$.

\section{Artostrobium miralestense (Campbell and Clark)}

(Plate 5, Figures 31-35; Plate 12, Figures 28-31)

Dictyocephalus miralestensis Campbell and Clark, 1944, p. 45, pl. 6, figs. 12-14.

Eucyrtidium tumidulum ? Bailey, Hays, 1965, p. 181, pl. 3, fig. 7. Eucyrtidium tumidulum Bailey (?), Kling, 1971, pl. 1, fig. 2.

Artostrobium miralestense (Campbell and Clark), Riedel and Sanfilippo, 1971, pl. 1H, figs 9-17; pl. 2I, figs. 9, 10; pl. 3E, fig. 12.

(?) Eucyrtidium tumidulum Bailey, 1856, p. 5, pl. 1, fig. 6.

\section{Genus LITHOMITRA Bütschli 1882}

Lithomitra lineata (Ehrenberg) group

(Plate 12, Figures 19, 20)

Lithocampe lineata Ehrenberg, 1838, p. 130 (partim); 1854, pl. 22, fig. 26 ; pl. 36 , fig. 16 .

Lithomitra lineata (Ehrenberg) group, Riedel and Sanfilippo, 1971, p. 1600 , pl. 1I, figs. $1-11$; pl. 2I, figs. 14-16; pl. 3E, fig. 14 .

\section{Genus SIPHOCAMPE Haeckel 1881}

Siphocampe corbula (Harting)

(Plate 5, Figures 22, 23; ? Plate 12, Figures 21-23)

Lithocampe corbula Harting, 1863, p. 12, pl. 1, fig. 21.

Siphocampe corbula (Harting), Nigrini, 1967 , p. 85, pl. 8, fig. 5 , pl. 9, fig. 3; Riedel and Sanfilippo, 1971, p. 1601, pl. 1H, figs. $18-25$.

\section{Genus SPIROCYRTIS Haeckel 1881}

\section{Spirocyrtis sp. aff. S. Scarlaris Haeckel}

(Plate 5, Figures 24-26)

Spirocyrtis scalaris Haeckel, 1887, p. 1509, pl. 76, fig. 14; Nigrini, 1967 , p. 88 , pl. 8 , fig. 7 , pl. 9 , fig. 4 .

Spirocyrtis $s p$. aff. S. scalaris Haeckel, Riedel and Sanfilippo 1971, pl. 1G, figs. $19-24$; pl. $2 \mathrm{H}$, fig. $3,15-18$. 


\section{REFERENCES}

Bailey, J. W., 1856. Notice of microscopic forms found in the soundings of the Sea of Kamtchatka. Am. J. Sci., Ser. 2. 22, 1.

Benson, R. N., 1964. Preliminary report on Radiolaria in Recent sediments of the Gulf of California. In Marine Geology of the Gulf of California - A Symposium. Am. Assoc. Petrol. Geologist Mem. 3. 398.

Bütschli, O., 1882. Beiträge zur Kenntnis der Radiolarienskelette, insbesondere der Cyrtida. Z. Wiss. Zool. 36, 485 .

Campbell, A. S., 1954. Radiolaria. In Treatise on Invertebrate Paleontology. R. C. Moore (Ed.). (Univ. Kansas Press and Geol. Soc. Am). Pt. D., Protista 3. 11.

Campbell, A. S. and Clark, B. L., 1944. Miocene radiolarian faunas from southern California. Geol. Soc. Am., Spec. Paper 51.76 p.

Casey, R. E., 1971. Radiolarians as indicators of past and present water masses. In The Micropaleontology of Oceans. B. M. Funnell and W. R. Riedel (Eds.). Cambridge (Cambridge Univ. Press). 331.

Clark, B. L. and Campbell, A. S., 1942. Eocene radiolarian faunas from the Mt. Diablo area, California. Geol. Soc. Am. Spec. Paper 39, 112 p.

Dogiel, V. A. and Reshetnyak, V. V., 1952. Materialy po radiolyariuam severo-zapadnoi chasti Tikhogo Okeana. Issle. Dalnevost. Morei SSR, no. 3. 5.

Dreyer, F., 1889. Die Pylombildungen in vergleichendanatomischer und entwicklungs-geschichtlicher Beziehung bei Radiolarien und bei Protisten überhaupt. Jena. Zietschr. Naturw. 23 (N. F. 16), 77.

Ehrenberg, C. G., 1838. Uber die Bildung der Kriedefelsen und des Kriedemergels durch unsichtbare Organismen. Kgl. Akad. Wiss. Berlin, Abh. Jahre 1838.

1844. Über 2 neue Lager von Gebirgsmassen aus Infusorien als Meeres-Absatz in Nord-Amerika und eine Vergleichung derselben mit den organischen KriedeGebilden in Europa und Afrika. Kgl. Preuss. Akad. Wiss. Berlin, Ber. Jahre 1844.

1847. Über die mikroskopischen kieselschaligen Polycystinen als mächtige Gebirgsmasse von Barbados und über das Verhältniss der aus mehr als 300 Neuen Arten bestehenden ganz eigenthümlichen Formengruppe jener Felsmasse zu den jetzt lebenden Thieren und zur Kriedebildung. Eine neue Anregung zur Erforschung des Erdlebens. Kgl. Preuss. Akad. Wiss. Berlin, Ber. Jahre 1847.

1854-1856. Mikrogeologie. Leipzig (Voss). 374 p. Fortsetzung (1956). $88 \mathrm{p}$.

1858. Kurze Characteristik der 9 neuen Genera und der 105 neuen Species des ägäischen Meeres und des Tiefgrundes des Mittel-Meeres. Kgl. Preuss. Akad. Wiss., Monatsber. Jahre 1858. 10.

, 1961. Die Tiefgrund-Verhältnisse des Oceans am Eingange der Davisstrasse und bei Island $\mathrm{K}$. Preuss. Akad. Wiss. Berlin, Monatsber. 1861, p. 275.

1872a. Mikrogeologische Studien als Zusammenfassung der Boebachtungen des kleinsten Lebens der Meeres Tiefgründe aller Zonen und dessen geologischen Einfluss. Kgl. Preuss. Akad. Wiss. Berlin, Monatsber. Jahre 1972.

1972b. Mikrogeologische Studien über das kleinste Leben der Meeres-Tiefgründe aller Zonen und dessen geologischen Einfluss. Kgl. Akad. Wiss. Berlin, Abh. Jahre 1972.
Goll, R. M. 1972. Leg 9 Synthesis, Radiolaria. In Hays, J. D., et. al., 1972. Initial Reports Deep Sea Drilling Project, Vol. IX. Washington (U. S. Government Printing Office).

Haeckel, E., 1960. Abbildungen und Diagnosen neuer Gattungen und Arten von lebenden Radiolarien des Mittelmeeres. Kgl. Preuss Akad. Wiss., Monatsber. Jahre 1860.

1862. Die Radiolarien, Eine Monographie. 572 p. 1881. Entwurf eines Radiolarien-Systems auf Grund von Studien der Challenger-Radiolarien. Jena. Z. Med. Naturwiss. 15, (new ser., 8), (3), 418.

1887. Report on the Radiolaria collected by H. M. S. Challenger during the years 1873-76. Rep. Voy. Challenger, Zool. 18. 1803 p.

Harting, P., 1863. Bijdrage tot de kennis der mikroscopische faune en flora van de Banda-Zee. Kon. Akad. Wetensch. Amsterdam, Verh. 10, 1.

Hays, J. D., 1965. Radiolaria and late Tertiary and Quaternary history of Antarctic seas. Am. Geophys. Union Antarc. Res. Ser. 5. 125.

1970. Stratigraphy and evolutionary trends of Radiolaria in North Pacific deep sea sediments. In J. D. Hays (Ed.) Geological Investigations of the North Pacific. Geol. Soc. Am. Mem. 126.

Hulsemann, K., 1963. Radiolaria in plankton from the Arctic drifting station T-3, including the description of three new species. Arctic Inst. Am. Tech. Paper, no. 13. 1 .

Ingle, J. C., Jr., 1967. Foraminiferal biofacies variation and the Miocene-Pliocene boundary in southern California. Bull. Am. Paleontology, vol. 52, no. 236, 217.

Biostratigraphy and Paleoecology of Early Miocene through Early Pleistocene benthonic and planktonic foraminifera, San Joaquin Hills - Newport Bay, Orange County, California. In Stinemeyer, E. H. (Ed.), The Pacific Coast Miocene Biostratigraphic Symposium, March 9-10, 1972, Bakersfield, California. Pacific Section, Soc. Economic Paleontologists and Mineralogists, 255.

Kling, S. A., 1971. Radiolaria. In Fischer, A. G., Heezen, B. C., et. al., 1971. Initial Reports of the Deep Sea Drilling Project, Volume VI. Washington (U.S. Government Printing Office). 1060.

Loeblich, A. R., Jr., and Tappan, Helen, 1961. Remarks on the systematics of the Sarkodina (Protozoa), renamed homonyms and new and validated genera. Proc. Biol. Soc. Washington. 74, 213.

Martin, G. C., 1904. Radiolaria. Maryland Geol. Survey (Miocene). 447

Moore, T. C., 1971. Radiolaria. In Tracey, J. I., Jr., Suggon, G. H., et al., 1971. Initial Reports Deep Sea Drilling Project, Volume VII. Washington (U.S. Government Printing Office). 727

Müller, Johannes, 1855 Über die im Hafen von Messina beobachteten Polycystinen. Kgl. Preuss. Akad. Wiss. Berlin, Ber. Jahre 1855, 671 .

1858. Über die Thalassicollen, Polycystinen und Acanthometren des Mittelmeeres. Abh. Kgl. Akad. Wiss. Berlin, Jahre 1858.1.

Nakaseko, Kojiro, 1963. Neogene Cyrtoidea (Radiolaria) from the Isozaki Formation in Ibaraki Prefecture, Japan. Osaka Univ. Sci. Rep. 12 (2), 165.

Nigrini, C., 1967. Radiolaria in pelagic sediments from the Indian and Atlantic Oceans. Bull. Scripps Inst. Oceanog. Univ. Calif. 11. 125 p. 
1968. Radiolaria from eastern tropical Pacific sediments. Micropaleontology. 14 (1), 51.

1970. Radiolarian assemblages in the North Pacific and their application to a study of Quaternary sediments in Core V20-130. In Geological Investigations of the North Pacific, J. D. Hays, (Ed.). Geol. Soc. Am., Mem. 126. 139.

1971. Radiolarian zones for the Quaternary of the equatorial Pacific Ocean. In The Micropaleontology of Oceans. Funnell, B. M. and Riedel, W. R. (Eds.). Cambridge (Cambridge Univ. Press). 443.

Petrushevskaya, M. G., 1967. Radiolyarii otryadov Spumellaria i Nassellaria antarkticheskoi oblasti (Antarctic spumelline and nasselline radiolarians). Issled. Fauny Morei 4 (12). Rez. biol. issled. Sov. Antarkt. Eksped. 1955-58. 3, 5 .

1971. Radiolyarii Nassellaria v planktone mirovogo okeana. (Radiolaria Nassellaria of the world ocean). Issled. Fauny Morei IX (XVII). Akad. Nauk., S.S.S.R., Izdat. "Nauka". 417 p.

Popofsky, A., 1908. Die Radiolarien der Antarktis (mit Ausnahme der Tripyleen). Deutsche Südpolar-Exped. 1901-1903. 10, (Zool. 2), (3), 183.

1912. Die Sphaerellarien des Warmwassergebietes. Deutsche Südpolar-Exped. 1901-1903. 13, (Zool., 5), (2), 73.

Reid, J. L., Jr., Roden, G. I., and Wyllie, J. G., 1958. Studies of the California Current System. Progress Report California Cooperative Oceanic Fisheries Investigations (January, 1958). 27.

Riedel, W. R., 1953. Mesozoic and Late Tertiary Radiolaria of Rotti. J. Paleontol. 27 (6), 805.

1958. Radiolaria in antarctic sediments. Rep. B.A.N.Z. Antarct. Res. Exped., ser. B. 6, pt. 10, 217. 1959. Oligocene and Lower Miocene Radiolaria in tropical Pacific sediments. Micropaleontology. 5 (3), 285 .

1967a. Some new families of Radiolaria. Proc. Geol. Soc. London. no. $1640,148$.

1967b. Subclass Radiolaria. In The Fossil Record. W. B. Harland et al. (Eds.). London (Geol. Soc. London). 291.

1971. Systematic classification of polycystine Radiolaria. In The Micropalaeontology of Oceans. B. M. Funnel and W. R. Riedel (Eds.). Cambridge (Cambridge Univ. Press). 649.

Riedel, W. R. and Sanfilippo, A., 1970. Radiolaria, Leg 4, Deep Sea Drilling Project. In Bader, R. G., Gerard, R. D., et al., 1970. Initial Reports of the Deep Sea Drilling Project, Volume IV. Washington (U.S. Government Printing Office). 503.

1971. Cenozoic Radiolaria from the western tropical Pacific, Leg 7. In Winterer, E. L., Riedel, W. R., et al., 1971. Initial Reports of the Deep Sea Drilling Project, Volume VII, Pt. 2. Washington (U.S. Government Printing Office). 1529.

Sanfilippo, Annika and Riedel, W. R., 1970. Post-Eocene "closed" theoperid radiolarians. Micropaleontology. 16 (4), 446 .

in press. Cenozoic Radiolaria (exclusive of theoperids, artostrobids and amphypyndacids) from the Gulf of Mexico, DSDP Leg X. In Worzel, J. L., et. al., in press. Initial Reports of the Deep Sea Drilling Project, Volume X. Washington (U.S. Government Printing Office).

Sanfilippo, Annika, Burckle, L. H., Martini, E., and Riedel, W. R., in press. Radiolarians, diatoms, silicoflagellates, and calcareous nannofossils in the Mediterranean Neogene Micropaleontology.

\section{PLATES}

Plates 1 through 12 constitute the synchronopticon with Plates 1 through 5 covering the Pliocene and Pleistocene, and Plates 6 through 12 covering the Miocene. Magnifications are $120 \mathrm{X}$ unless otherwise stated. Samples used for each of the ten different horizons are, except for the few cases otherwise noted, as follows:

Artostrobium miralestense Zone: 173-1-2 (102-104 cm) Axoprunum angelinum Zone: 173-3-3 (106-108 cm). Eucyrtidium matuyamai Zone: 173-6-2 $(55-57 \mathrm{~cm})$

Lamprocyrtis heteroporos Zone: 173-9-3 (57-59 cm)

Unzoned (lower Pliocene): 173-14-2 (92-94 cm)

Unzoned (upper Miocene): 173-16-1 (70-72 cm)

Ommatartus antepenultimus Zone: 173-19-4 $(57-59 \mathrm{~cm})$

Cannartus petterssoni Zone: $173-22-3(57-59 \mathrm{~cm})$

Dorcadospyris alata Zone: $173-29-1(57-59 \mathrm{~cm})$

Calocycletta costata Zone: $173-31-1(128-130 \mathrm{~cm})$

Samples used for Plates 13 through 15 are noted for each figure. Magnifications are 240X unless otherwise noted.

Slide numbers, abbreviated Sl., refer to different individual slides. These numbers are followed by England Finder coordinates; the slide label was placed to be to the right as the England Finder was placed on the stage to read normally (label to the left) unless the letter $L$ indicates the opposite position. Abundances in the synchronopticon are abbreviated: $r$ - rare, $f$ - few, $c$ - common, $a$ - abundant, + less than three specimens per slide.

Some illustrations in Plate 15 are taken from Scripps Institution of Oceanography cores, abbreviated S.I.O. 


\section{PLATE 1}

Figures 1-4 Polysolenia sp.

1.S1. 1, F 57/1

2. S1. $1, \mathrm{X} 41 / 2$

3. S1. $2, X 8 / 3, \mathrm{~L}$

4. S1. 1, U 61/0

Figures 5-10 Sphaeropyle langii

5. S1. 1, D 41/3

6. S1. 1, B 37/4

7. S1. $1, \mathrm{X} 73 / 1$

8. S1. 1, T 39/4

9. S1. 1, J $31 / 1$

10. S1. $1,055 / 4$

Figures 11, 12 Sphaeropyle robusta

11. S1. 2, G 23/4

12. S1. 1, Y 42/1

Figures 13-16 Axoprunum angelinum

13. S1. 1, G 29/3

14. S1. 1, K 30/1

15. S1. 1, H 41/2

16. S1. 1, T $69 / 0$

Figures 17-20 Stylacontarium acquilonium

17. S1. 1, Y 66/1

18. S1. 1, C 57/0

19. S1. 1, T 57/2

20. S1. 1, R $66 / 4$

Figures 21-25 Saturnalis circularis

21. S1. 1, D 65/4

22. S1. 1, U $42 / 1$

23. S1. 1, A $47 / 3$

24. S1. 1, D $43 / 3$

25. S1. 1 , F $35 / 2$ 
PLATE 1

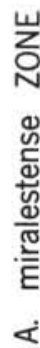
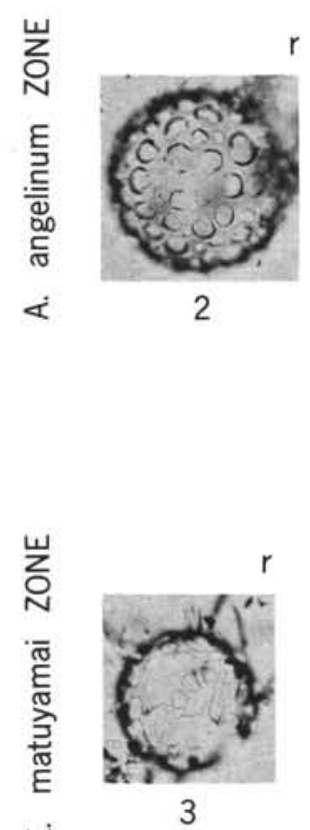

ن

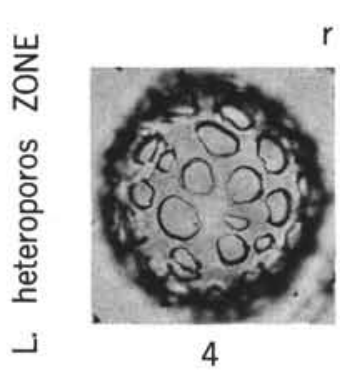

음

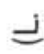

을

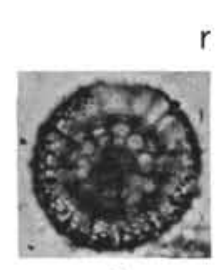

5

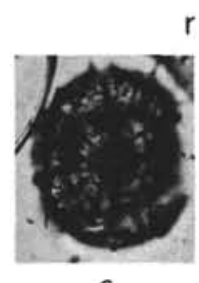

6

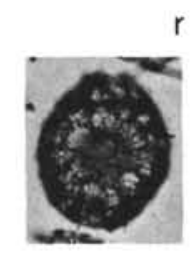

8

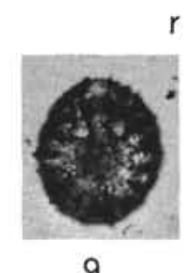

9

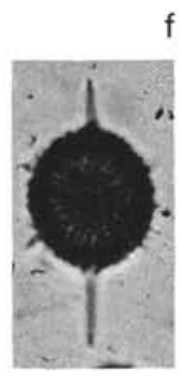

13

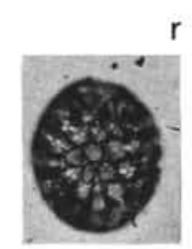

10

7

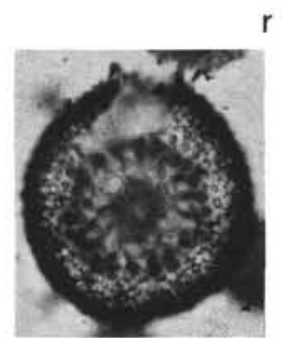

11

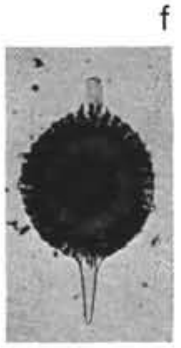

14

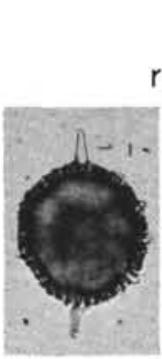

15

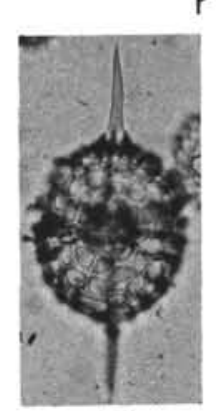

17

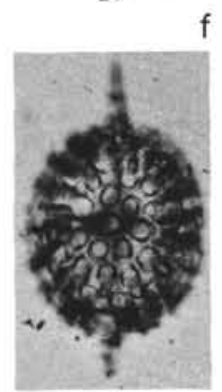

18

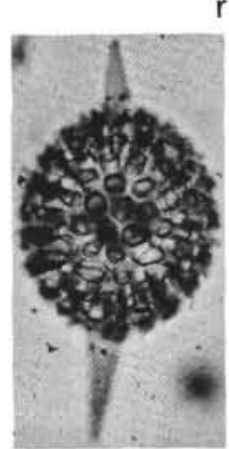

19

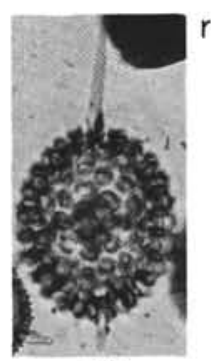

20

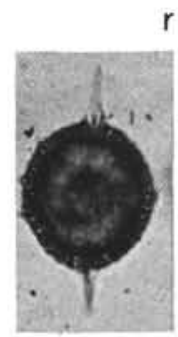

16

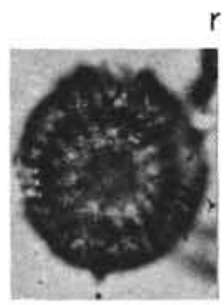

12

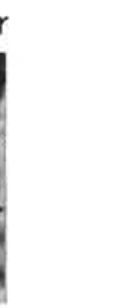

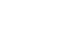

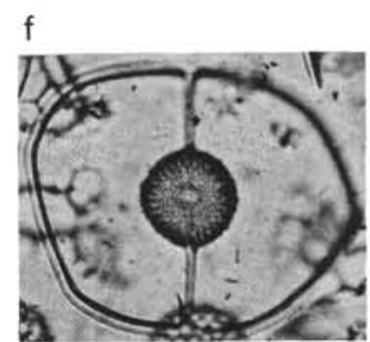

21
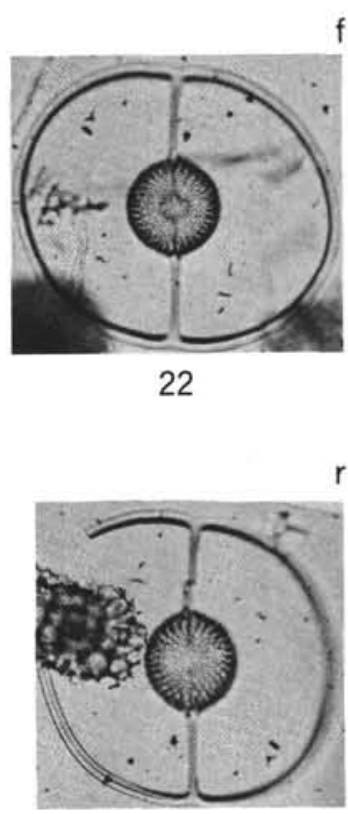

23

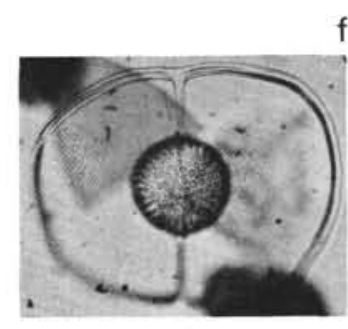

24 
PLATE 2

Figures 1-3 Xiphospira sp. cf. $X$. circularis

1. S1. $1, \mathrm{~J} 28 / 3$

2. S1. $1,039 / 0$

3. S1. $1, \mathrm{H} 55 / 1$

Figures 4-6 Spongotrochus glacialis

4. S1. 1, F 36/1, 69X

5. S1. 2, Y $62 / 2,84 \mathrm{X}$

6. S1. $1,045 / 1,69 X$

Figure 7 Litheliidae, gen. et. sp. indet.

7. S1. 2, V $35 / 1$

Figures 8-14 Acanthodesmiidae, gen. et. spp. indet.

8. S1. $1, \mathrm{~W} 33 / 0$

9. S1. $1, \mathrm{R} 51 / 0$

10. S1. 1, L 26/4

11. S1. 1, U 39/1

12. S1. 1. K. $65 / 0$

13. S1. $2, \mathrm{~K} 44 / 4$

14. S1. 2, B $21 / 2$

Figures 15-19 Peripyramis circumtenta

15. S1. 1, M 25/1

16. S1. 1, N 27/1

17. S1. $1,047 / 0$

18. S1. 1, H $58 / 1$

19. S1, 2, X 26/0

Figures 20-23 Bathropyramis woodringi

20. S1. $1, \mathrm{H} 31 / 2$

21. S1. $1, \mathrm{P} 38 / 1$

22. S1. $1, \mathrm{P} 45 / 1$

23. S1. 1, Q 35/2 
PLATE 2
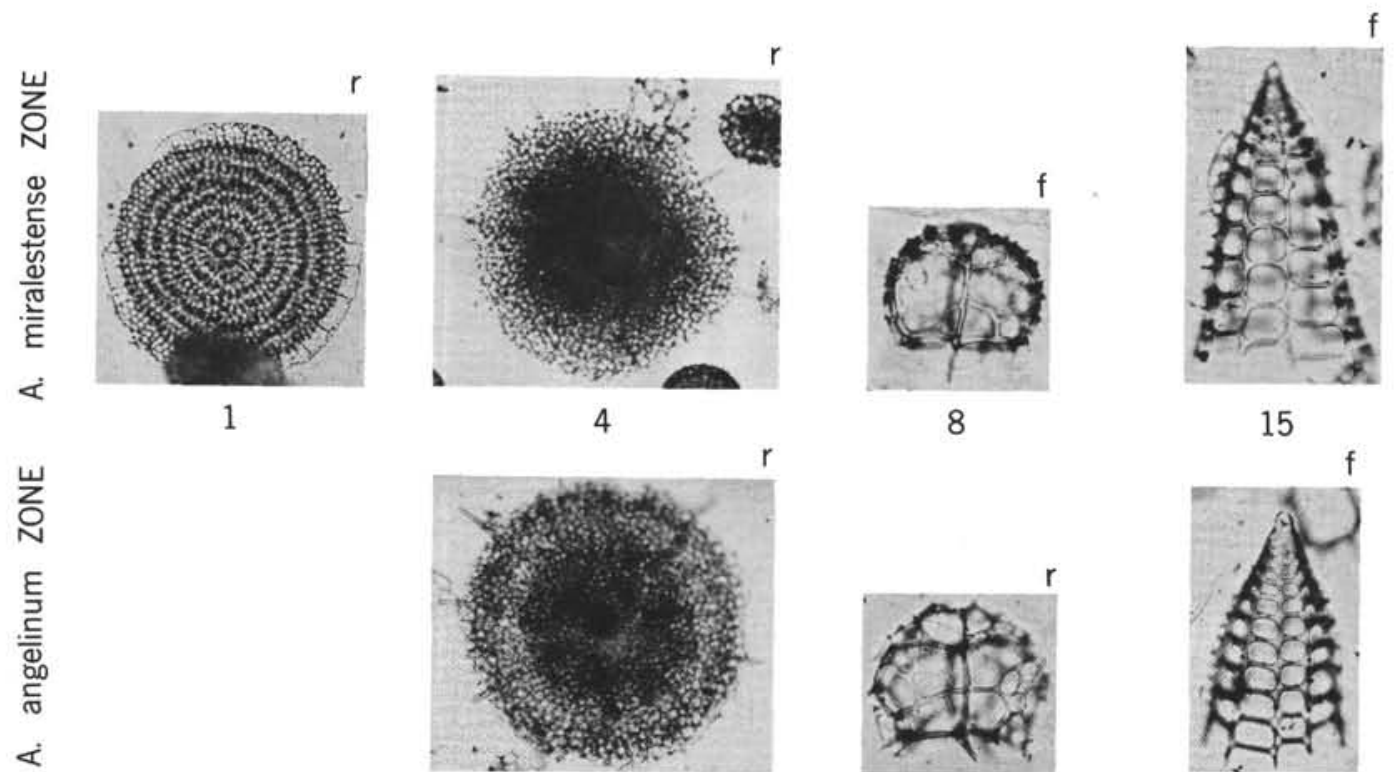

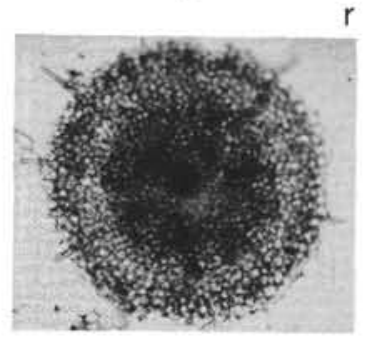

5
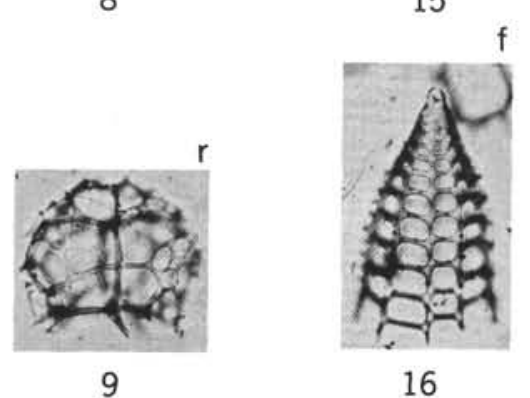

16
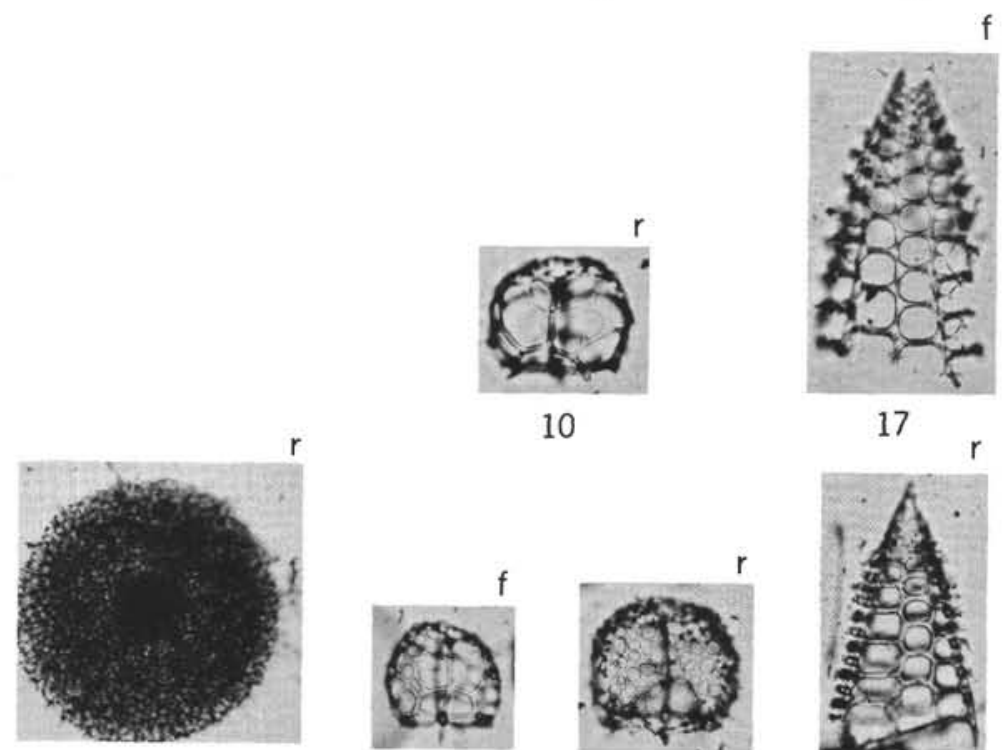

6

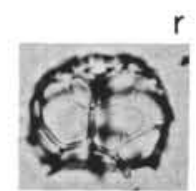

10
17
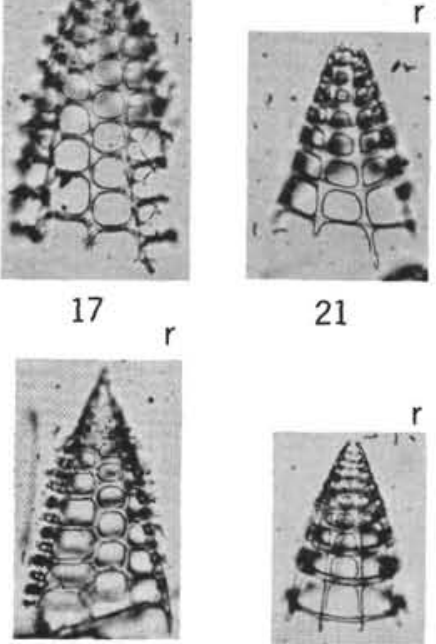

18
21

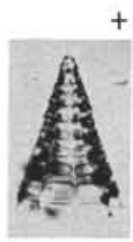

20

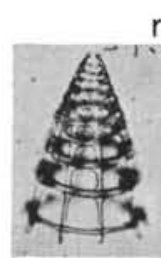

22
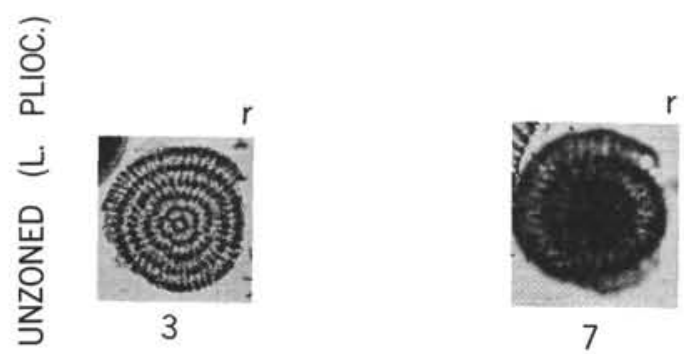

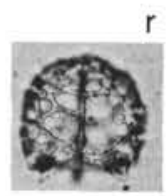

13

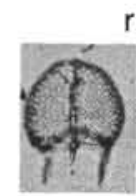

14

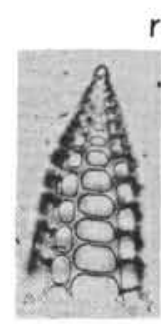

19

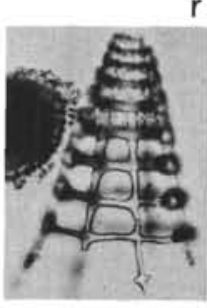

23 


\section{PLATE 3}

Figures 1-4 Cornutella profunda

1. S1. 1, T $67 / 4$

2. S1. $1, \mathrm{U} 25 / 3$

3. $11.2, \mathrm{~N} 23 / 4$

4. S1. $2, \mathrm{Y} 42 / 3$

Figures 5-8 Theocalyptra davisiana(?)

5. S1. $1, \mathrm{C} 64 / 0$

6. S1. $1, \mathrm{C} 63 / 0$

7. S1. $1, \mathrm{M} 53 / 3$

8. S1. 2, Q $21 / 4$

Figures 9-12, 28 Theocalyptra davisiana

9. S1. 1, U 29/2

10. S1. $1,029 / 3$

11. S1. $1, \mathrm{P} 37 / 0$

12. S1. $1, \mathrm{Q} 35 / 4$

28. S1. 1, G 31/0

Figures 13-16 Theocalyptra sp.

13. S1. $1,027 / 2$

14. S1. $1, \mathrm{Y} 28 / 1$

15. S1. 1, R $25 / 4$

16. S1. 1, L $45 / 4$

Figures 17-22 Clathrocyclas sp.

17. S1. 1, F $25 / 4$

18. S1. 1, F 29/3

19. S1. 1, T $28 / 3$

20. S1. 1, R $25 / 3$

21. S1. 2, E $48 / 0$

22. S1. $1, \mathrm{~N} 31 / 0$

Figures 23, 24 Stichophormis novena Haeckel 1887

23. S1. 1, P $66 / 0$

24. S1. 1, D 32/0

Figures 25-27 Stichopera pectinata group

25. S2. 2, N 63/3

26. S1. $1, \mathrm{D} 64 / 0$

27. S1. 1, G $31 / 0$ 
PLATE 3
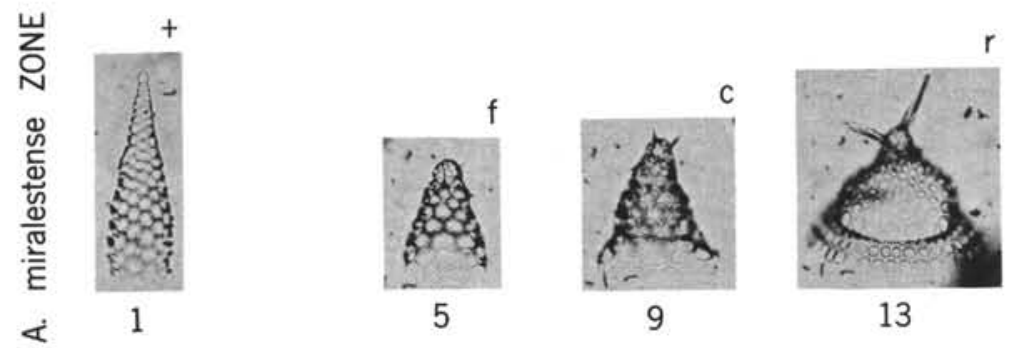

13

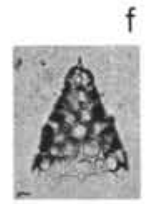

6

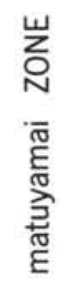

ن
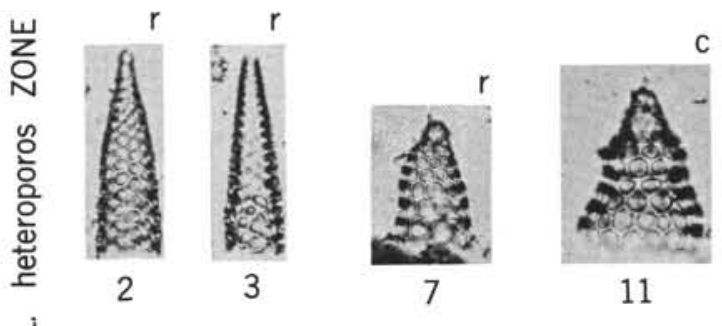

11
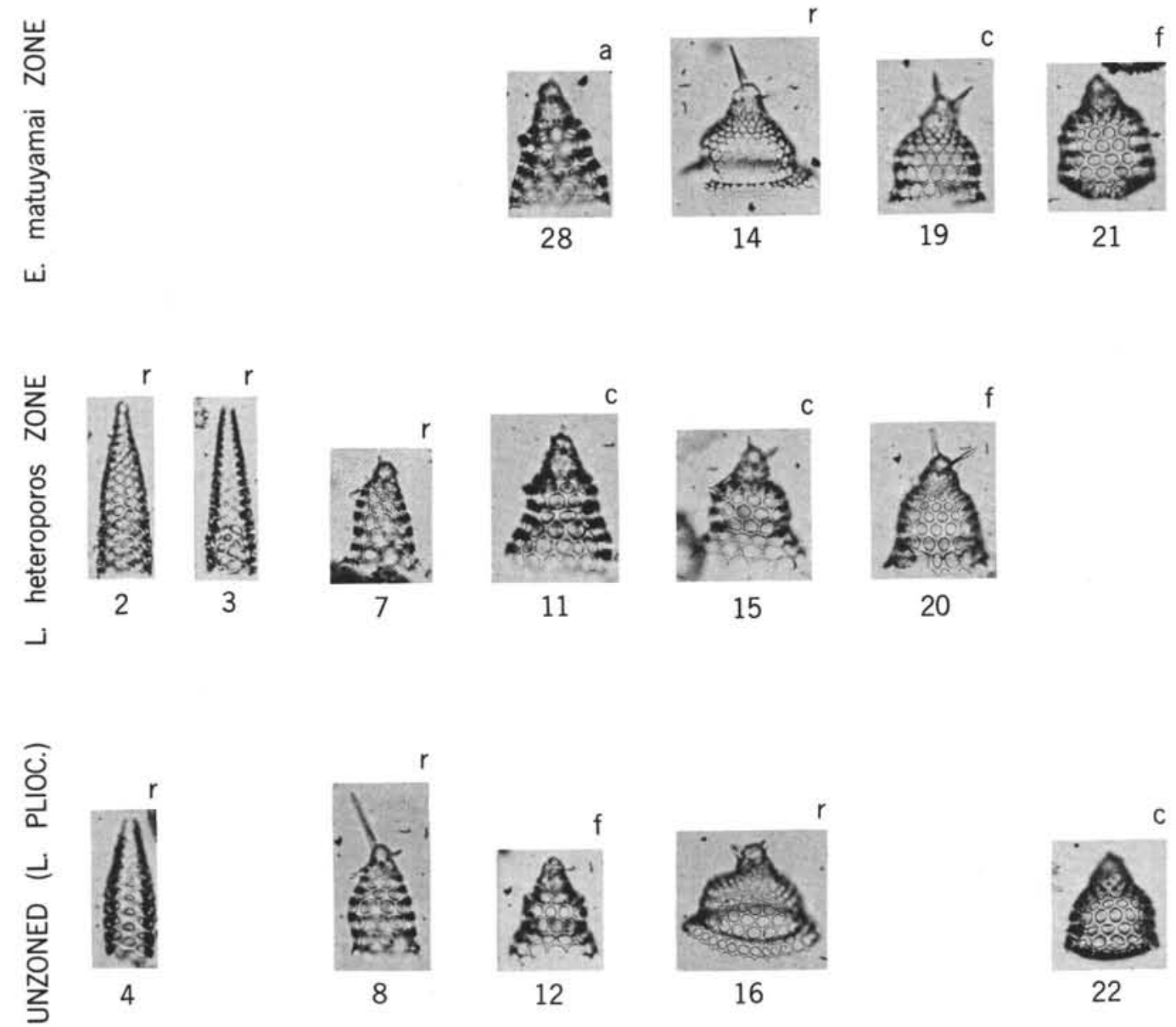

20
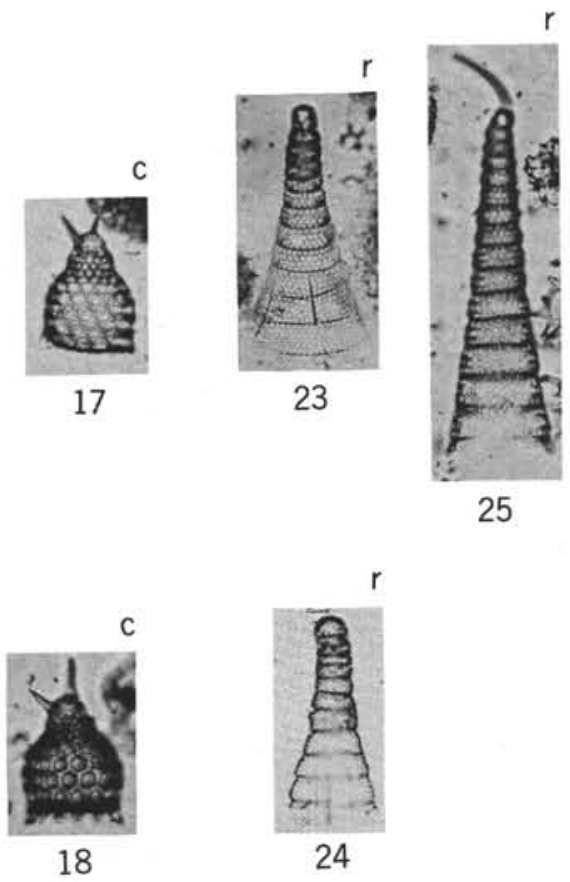

8

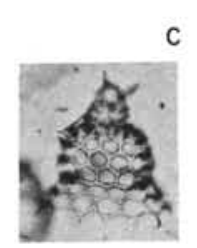

15
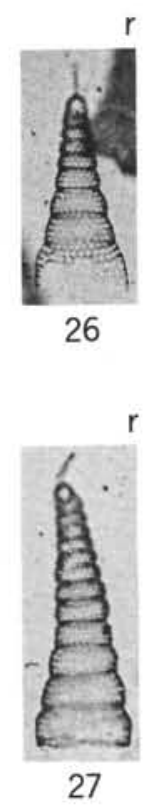


\section{PLATE 4}

Figures 1-4 Pterocanium korotnevi

1. S1. 1, U $38 / 0$

2. S1. $1, \mathrm{P} 28 / 3$

3. S1. $1, \mathrm{H} 70 / 2$

4. S1. $2, \mathrm{~S} 45 / 0$

Figures 5-8 Pterocanium trilobum

5. S1. 1, G 37/0

6. S1. $1, \mathrm{H} 30 / 0$

7. S1. $1, \mathrm{~V} 33 / 1$

8. S1. 1, Y $29 / 1$

Figures 9,10 Lychnocanoma sp. cf. L. grande

9. S1. 1, X 26/1

10. S1. $2, \times 27 / 1$

Figures 11-15 Dictyophimus crisiae

11. S1. 1, T 34/1

12. S1. 1 , E $24 / 0$

13. S1. 1, B $56 / 0$

14. S1. $2, \mathrm{G} 38 / 0$

15. S1. 1, H 39/3

Figures 16,18,19 Eucyrtidium calvertense

16. S1. 1, U 37/0

18. S1. 1, M $27 / 0$

19. S1. 1, J $68 / 2$

Figure $17 \quad$ Eucyrtidium matuyamai

S1. 2, J $28 / 4$

Figures 20-23 Eucyrtidium acuminatum

20. S1. $1, \mathrm{~K} 40 / 0$

21. S1. $1, \mathrm{Y} 60 / 3$

22. S1. $1, \mathrm{H} 43 / 0$

23.S1. $1,063 / 3$

Figures 24-26 Lipmanella dictyoceras

24. S1. 1, F 26/0

25. S1. 1, T 44/1

26. S1. 1, Y $64 / 0$

Figure 27 Stichocorys peregrina

173-14-3 (57-59), S1. 1, H 63/4 
岁
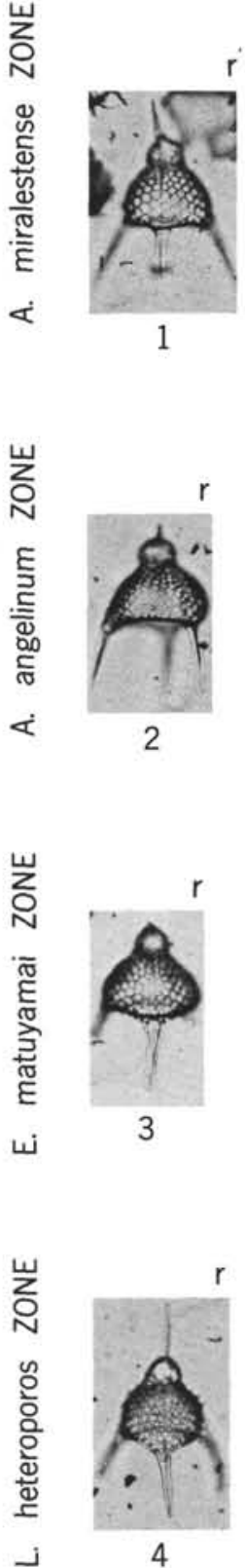

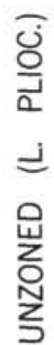

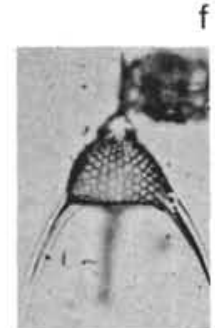

5

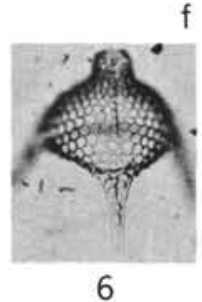

6
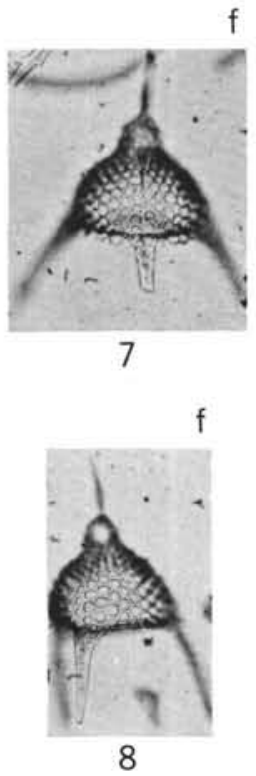

PLATE 4
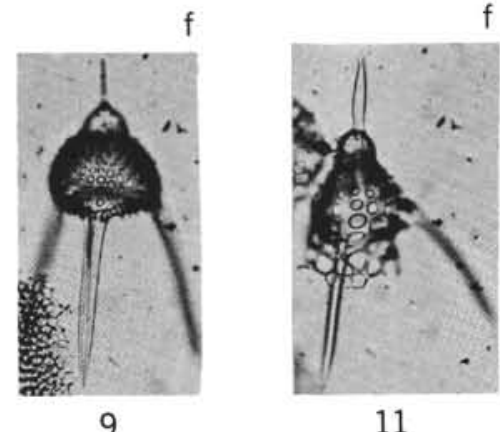

11

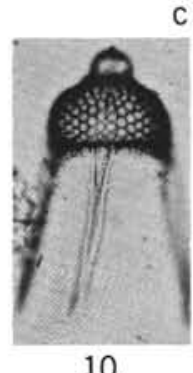

10

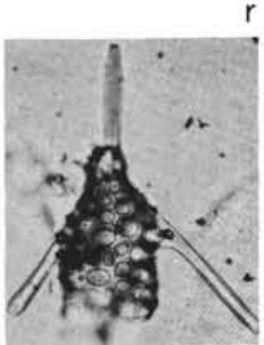

12

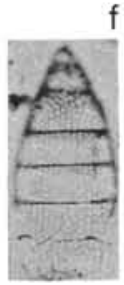

20

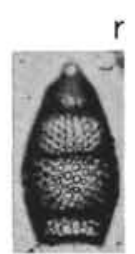

16

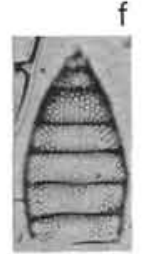

21

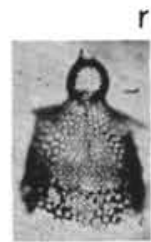

25

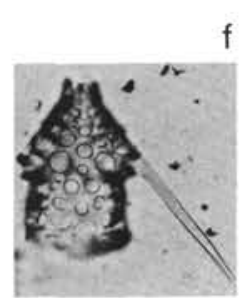

13

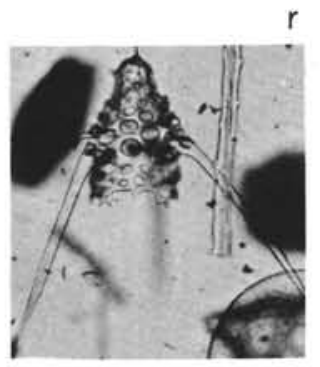

14

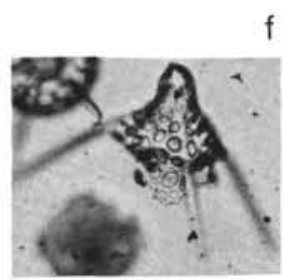

15

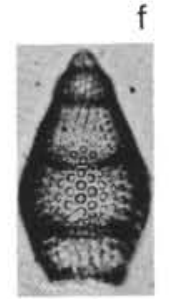

18

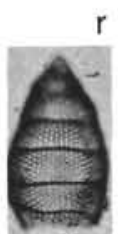

22

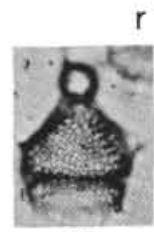

26 


\section{PLATE 5}

Figures 1-6 Carpocaniidae, gen. et spp. indet

$$
\begin{aligned}
& \text { 1. S1. 1, S 54/0 } \\
& \text { 2. S1. 1, L } 49 / 0 \\
& \text { 3. S1. 1, F } 49 / 1 \\
& \text { 4. S1. 2, K } 23 / 2 \\
& \text { 5. S1. 1, F } 41 / 2 \\
& \text { 6. S1. } 1 \text {, T } 27 / 1
\end{aligned}
$$

Figure $7 \quad$ Lamprocyclas maritalis polypora Nigrini 1970 S1. 2, U 32/2

Figure $8 \quad$ Theocorythium vetulum Nigrini 1971(?) S1. 2, Y $29 / 0$

Figures 9-11 Theoconus zancleus

9. S1. 1, N 28/3

10. S1. 1, Q $25 / 0$

11. S1. 2 , R $54 / 4$

Figures 12-14 Lamprocyrtis(?) hannai

12. S1. 1, D $65 / 4$

13. S1. $1, \mathrm{G} 38 / 2$

14. S1. 1, R 23/0

Figures 15,16 Lamprocyrtis haysi

15. S1. $1, \mathrm{~L} 52 / 3$

16. S1. 1, H $22 / 4$

Figures 17,18 Lamprocyrtis neoheteroporos

17. S1. $1,024 / 0$

18. S1. $1, \mathrm{U} 48 / 0$

Figures 19-21 Lamprocyrtis heteroporos

19. S1. 1, Y 27/1, L

20. S1. 1, Q 26/0

21. S1. 1, X 26/0

Figures 22, 23 Siphocampe corbula

22. S1. 1, V 55/1

23. S1. 1, B $40 / 3$

Figures 24-26 Spirocyrtis sp. aff. S. scalaris

24. S1. 1, T 53/0

25. S1. 2, Y $42 / 2$

26. S1. 2 , T $27 / 3$

Figures 27-30 Artostrobium auritum group

27. S1. 1, J 59/0

28. S1. 1, D 36/1

29. S1. 1, V $35 / 1$

30. S1. $1, \mathrm{C} 41 / 2$

Figures 31-35 Artostrobium miralestense

31. S1. 1, D 23/1

32. S1. 1, G 25/3

33. S1. 2, D 44/0

34. S1. 1, Q 31/1

35. S1. 1, U 25/2 
PLATE 5
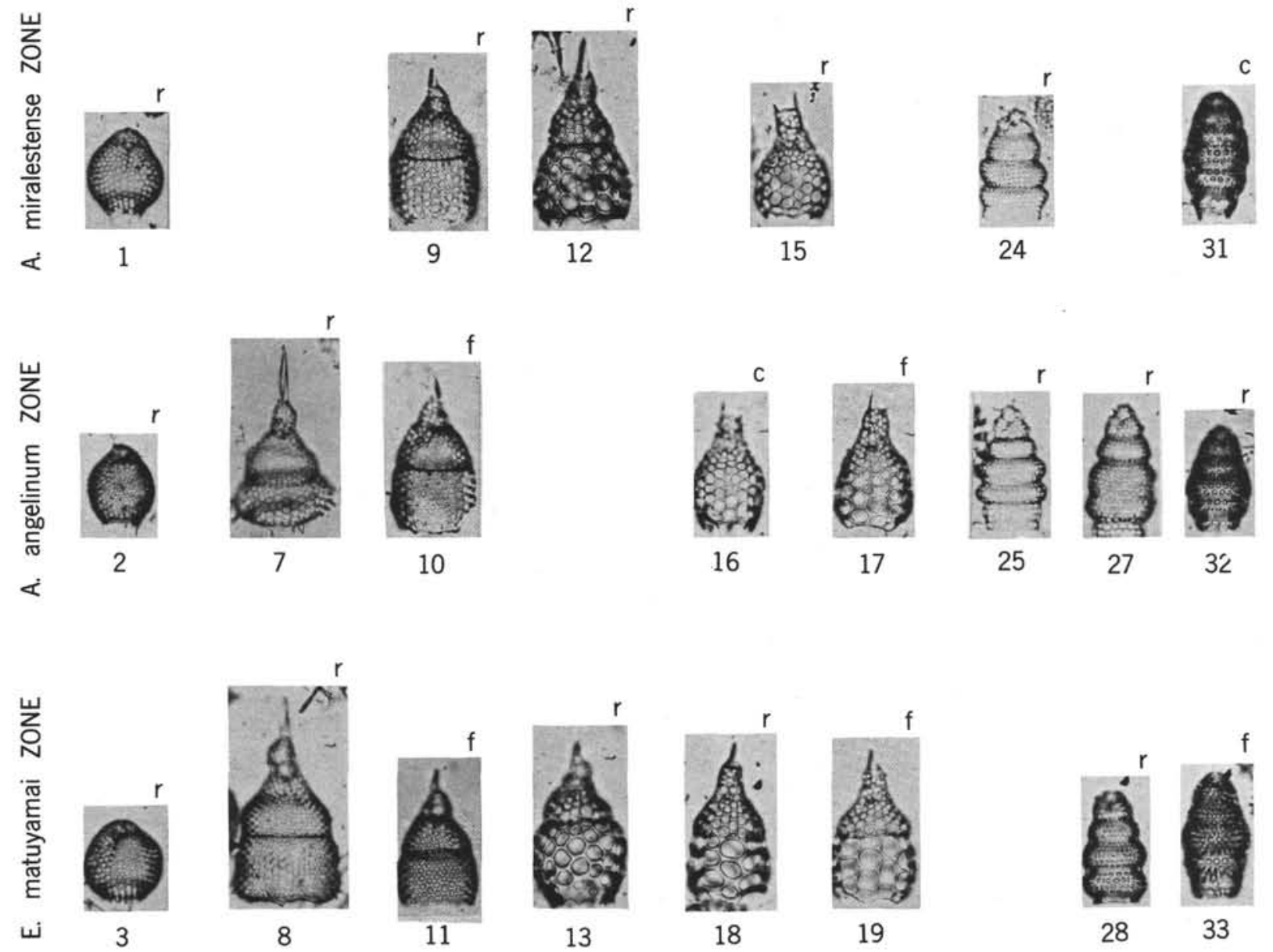

13

18

19
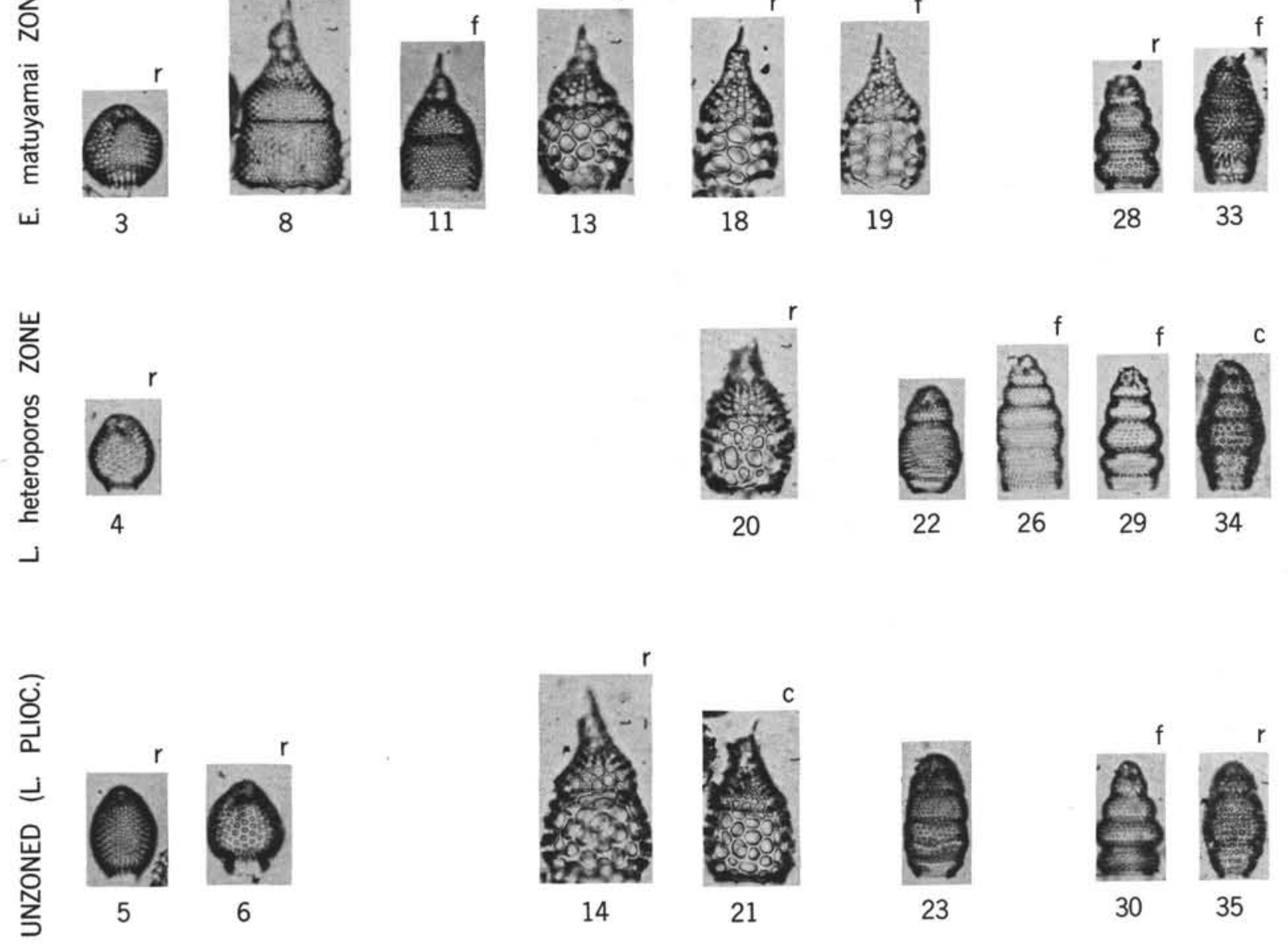


\section{PLATE 6}

Figures 1-3 Polysolenia sp.

1. S1. 1, C $22 / 4$

2. S1. 1, C $20 / 0$

3. S1. $2, \mathrm{G} 33 / 0$

Figures 4-8 Collosphaera spp.

4. S1. 1, C $21 / 2$

5. S1. $1, \mathrm{~V} 57 / 1$

6. S1. 1, U $52 / 0$

7. S1. $1, \mathrm{P} 31 / 4$

8. S1. $1, \mathrm{~V} 66 / 3$

Figures 9-13 Sphaeropyle robusta

9. S1. $1, \mathrm{~S} 63 / 3$

10. S1. $2, \mathrm{~S} 35 / 0$

11. S1. $1, \mathrm{M} 40 / 0$

12. S1. $1, \mathrm{C} 53 / 0$

13. S1. 1, C $38 / 2$

Figures 14-18 Axoprunum angelinum

14. S1. 2, U 34/1

15. S1. $2, \mathrm{X} 46 / 3$

16. S1. 1, R $23 / 0$

17. S1. 2 , C $31 / 0$

18. S1. 1, J 36/4

Figures 19-23 Stylacontarium sp. aff. S. bispiculum

19. S1. 1, A $57 / 0$

20. S1. $2, \mathrm{R} 48 / 4$

21. S1. $1, \mathrm{M} 31 / 0$

22. S1. $2, \mathrm{X} 71 / 0$

23. S1. 2 , J $22 / 0$ 


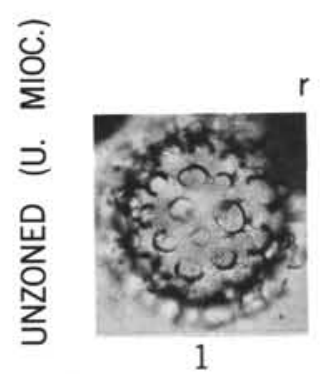

닝

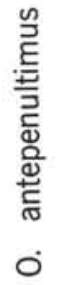

닝

흠

$\dot{0}$
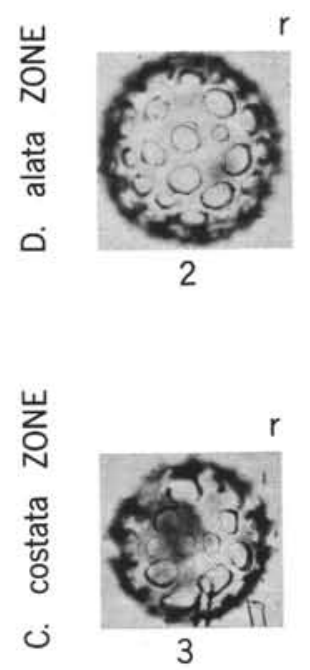
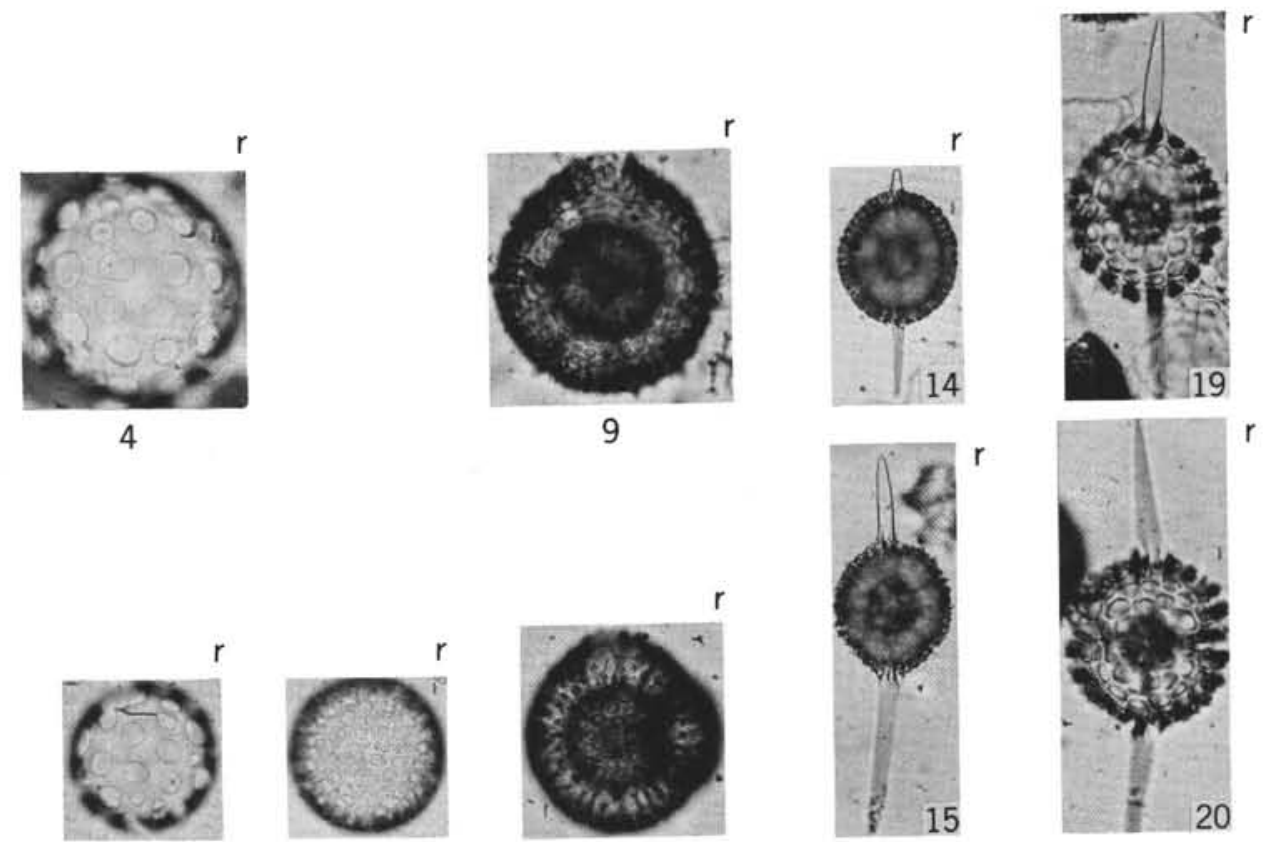

5
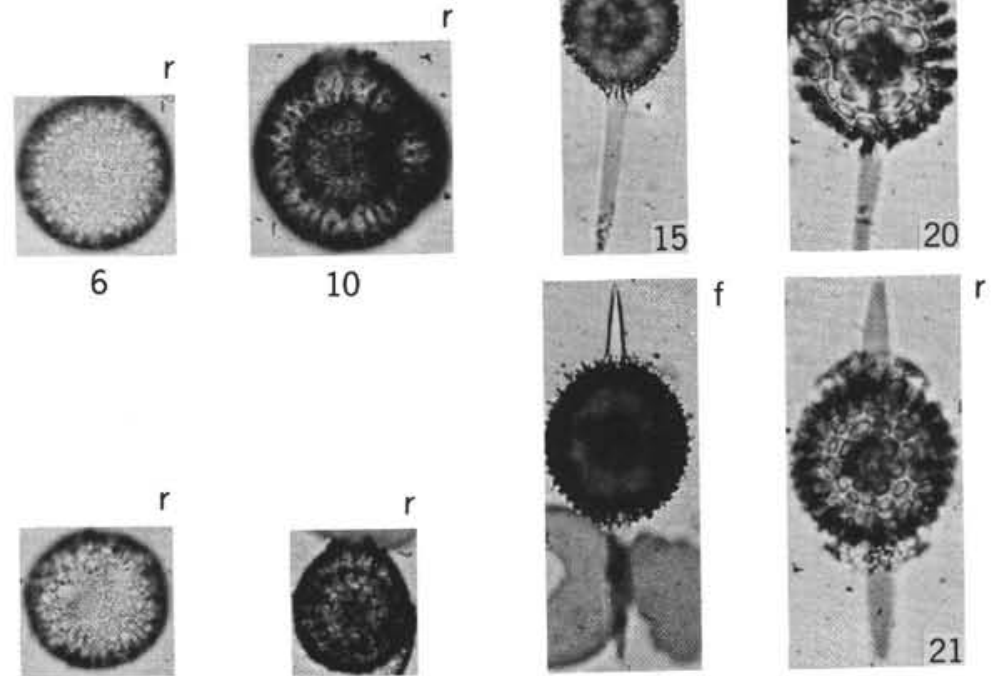

7

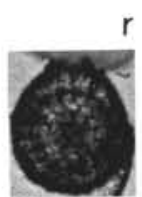

11

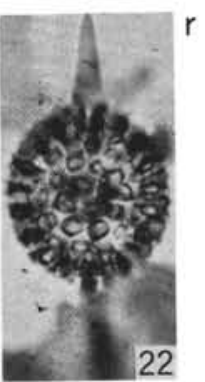

8

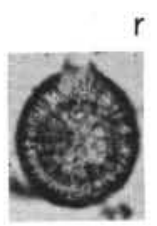

12
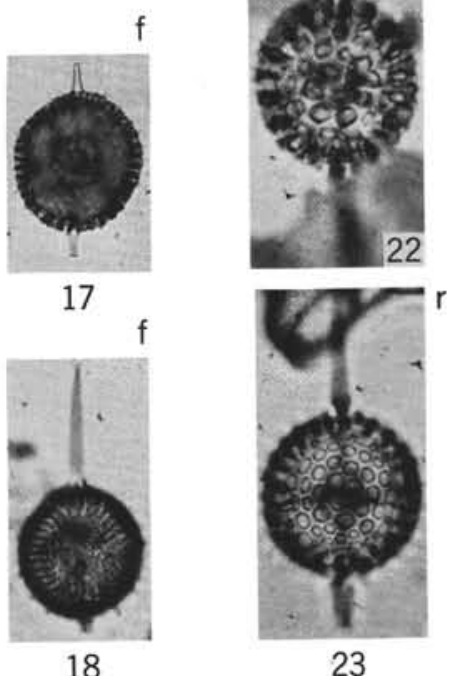

13

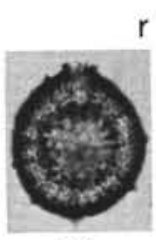


PLATE 7

Figures 1-5. Saturnalis circularis

1. S1. 1, E 37/1

2. S1. $1, \mathrm{C} 64 / 2$

3. S1. $1, \mathrm{Y} 40 / 1$

4. S1. 1, B $23 / 4$

5. S1. 1, T $26 / 3$

Figure 6

Ommatartus hughesi

S1. 1, Y 8/2, L

Figure 7

Cannartus laticonus

S1. 2, N 52/2

Figure $8 \quad$ Cannartus petterssoni

S1. 2, L 22/1

Figure 9 Cannartus mammiferus

S1. 1, 0 24/1

Figure $10 \quad$ Cannartus violina

S1. 2, M 56/4

Figures 11-17 Xiphospira sp. cf. X. circularis

11. S1. 2, E 52/4

12. S1. 2 , T $58 / 0$

13. S1. 1, R 36/1

14. S1. $1, \mathrm{H} 44 / 3$

15 S1. 1, J $38 / 0$

16. S1. $1, \mathrm{U} 42 / 2$

17. S1. $1, \mathrm{C} 45 / 1$

Figures 18-22 Spongocore puella

18. S1. 1, V 33/4

19. S1. 1, E $55 / 3$

20 S1. 1, W $36 / 0$

21. S1. 1, V 66/1

22. S1. $1, \mathrm{~S} 32 / 1$

Figures 23-25. Spirema sp.

23. S1. 1, E $54 / 0$

24. S1. 2, Y $69 / 3$

25. S1. 2, D 52/4 
PLATE 7
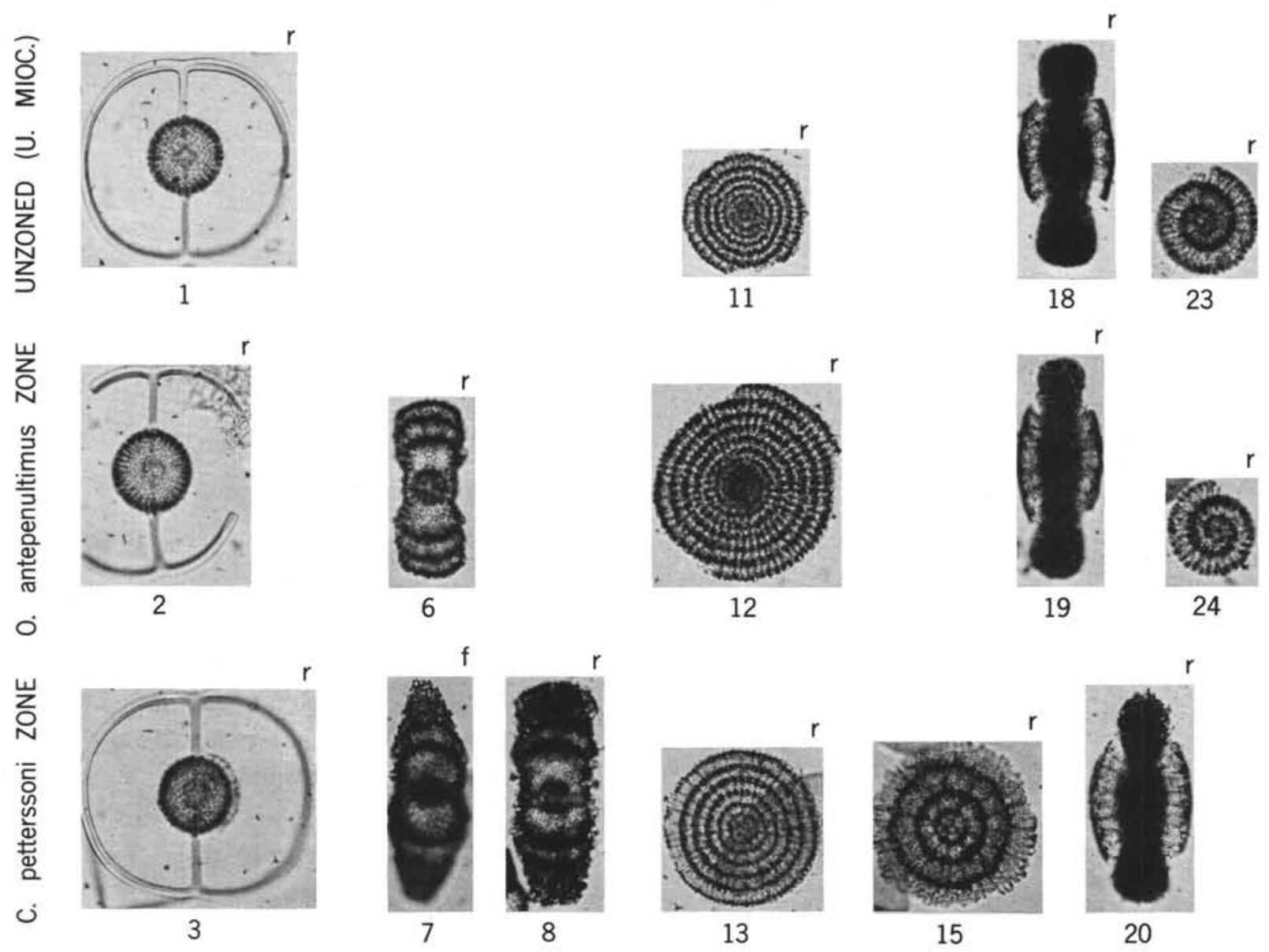

15
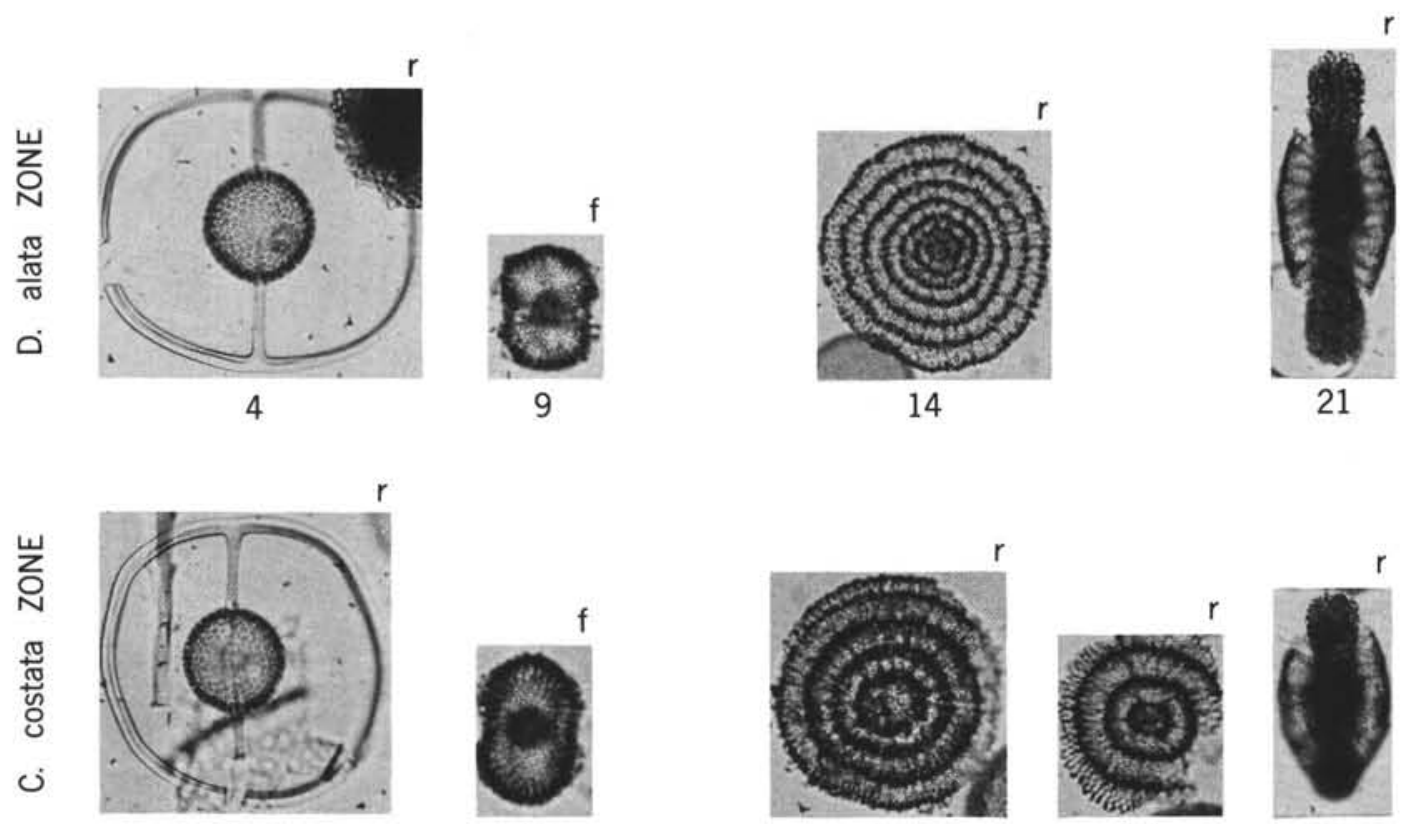

5
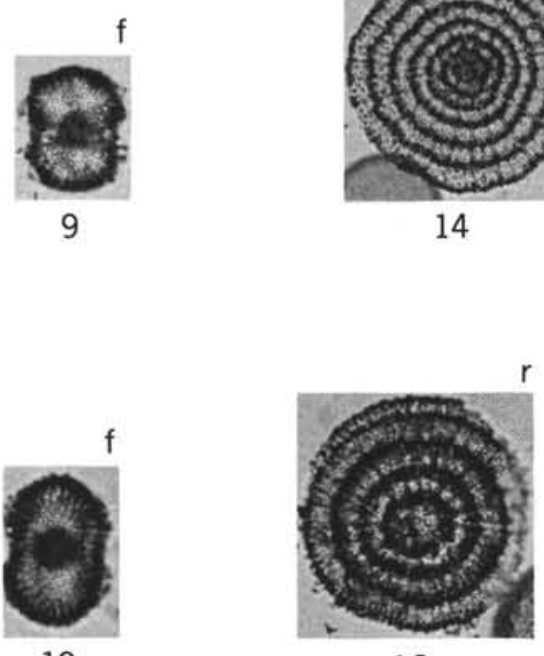

16
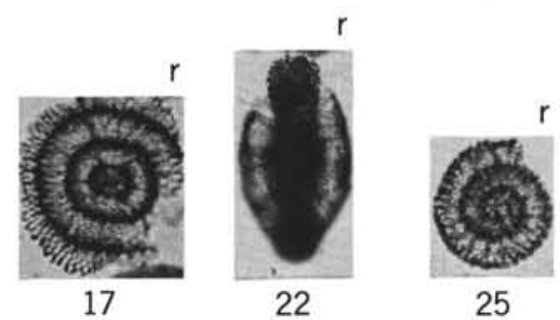


\section{PLATE 8}

Figures 1-26 Acanthodesmiidae, gen. et spp. indet.

1. S1. 1, S 22/0

2. S1. 2, D $25 / 2$

3. S1. $1, \mathrm{~V} 27 / 2$

4. S1. 2, T $42 / 4$

5. $\mathrm{S} 1,2, \mathrm{~K} 55 / 0$

6. S1. $1, \mathrm{~T} 23 / 0$

7. S1. 1 , T $54 / 2$

8. S1. $1, \mathrm{C} 53 / 1$

9. S1. 1, J 20/2

10. S1. 2, M 22/0

11. S1. 1, J 26/3

12. S1. 1, B $25 / 4$

13. S1. $2, \mathrm{~V} 66 / 0$

14. S1. 1, P 27/4

15. S1. 1, V 22/4

16. S1. $1,041 / 3$

17. S1. $1, \mathrm{H} 64 / 4$

18. S1. $2, \mathrm{~N} 45 / 0$

19. S1. 1, M $21 / 0$

20. S1. 2, E $33 / 3$

21. S1. $2, \mathrm{X} \mathrm{22/4}$

22. S1. 1, U 20/0

23. S1. 2, M $27 / 4$

24. S1. 1, T 27/2

25. S1. 1, X 23/4

26. S1. 1, P 63/1

Figure 27 Dorcadospyris simplex

S1. 1, L 22/0, 84X 
PLATE 8
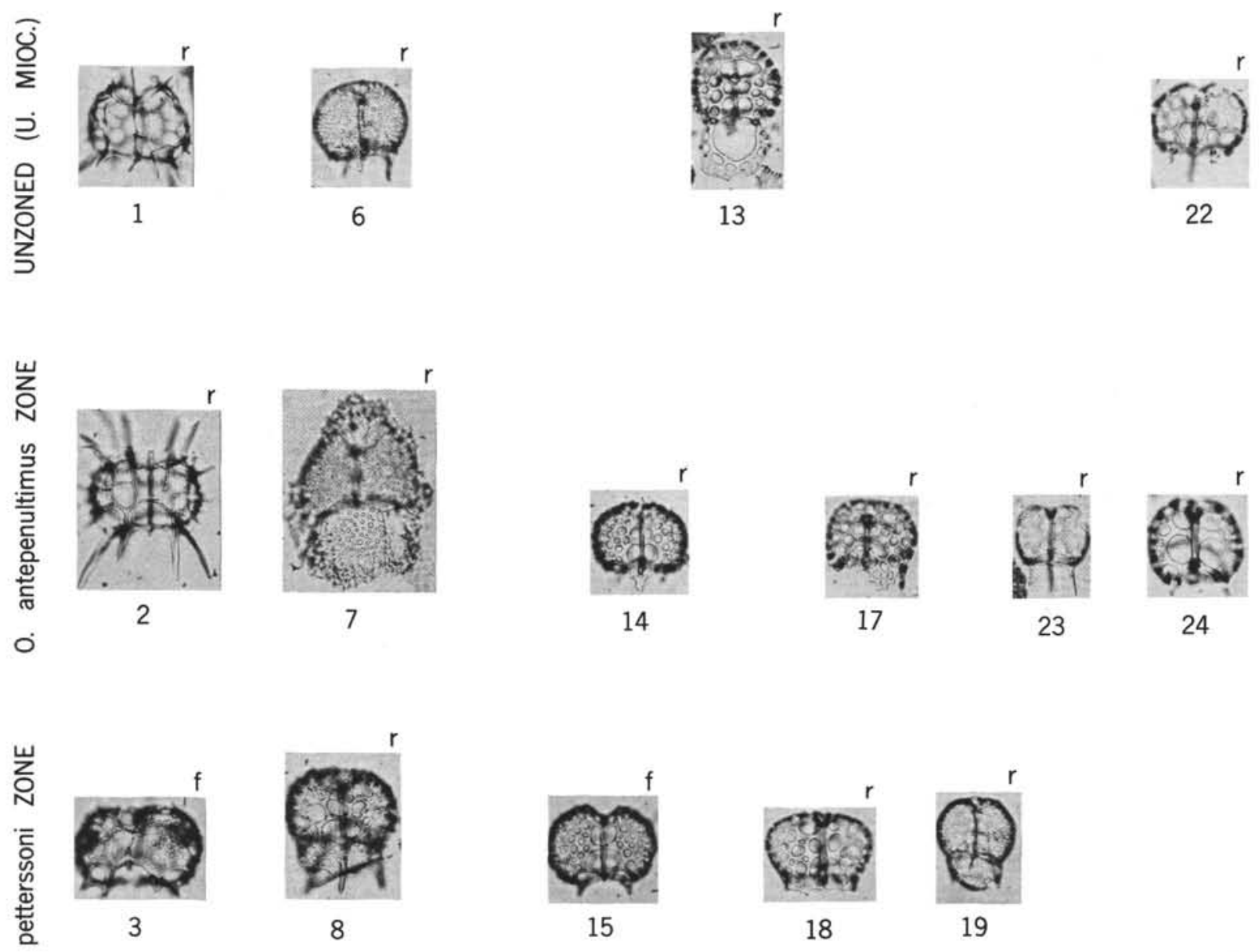

ن
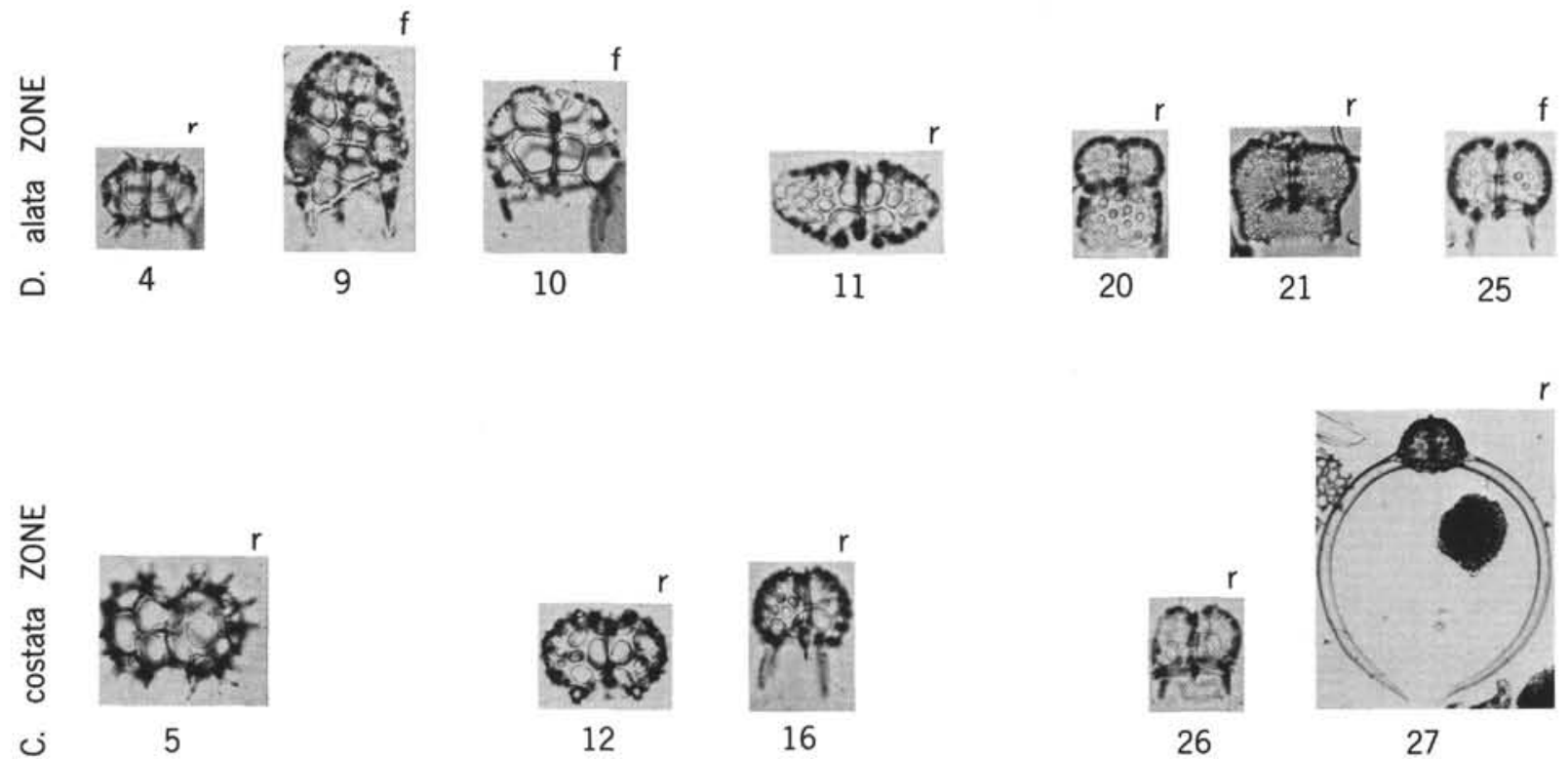


\section{PLATE 9}

Figures 1-3 Peripyramis circumtexta

1. S1. $1,063 / 1$

2. S1. $1,053 / 1$

3. S1. 1, Q 51/1

Figures 4,6 Bathropyramis woodringi(?)

4. S1. 1, M 61/2

6. S1. 1, L 20/2

Figures 5,7 Bathropyramis woodringi

5. S1. $2, \mathrm{X} 32 / 3$

7. S1. $2, \mathrm{H} 42 / 2$

Figures 8-17 Cornutella profunda
8. S1. 1, F 49/3
9. S1. $1, \mathrm{~V} 49 / 0$
10. S1. $2, \mathrm{~S} 41 / 1$
11. S1. $2, \mathrm{H} 58 / 0$
12. S1. $1,024 / 0$
13. S1. $1, \mathrm{E} 45 / 1$
14. S1. $1,025 / 1$
15. S1. $2, \mathrm{G} 41 / 0$
16. S1. $2, \mathrm{P} 36 / 3$
17. S1. 1, M $25 / 3$

Figures 18-22 Theocalyptra sp.

18. S1. 2, V 33/0

19. S1. $1, W 42 / 4$

20. S1. 1, M 20/1

21. S1. 2, Z 29/0

22. S1. 2 , T $50 / 0$

Figures 23-25 Clathrocyclas cabrilloensis

23. S1. $1, \mathrm{C} 65 / 2$

24. S1. $1, \mathrm{R} 25 / 0$

25. S1. $1, \mathrm{M} 65 / 2$

Figures 26-31 Clathrocyclas spp

26. S1. 1, E 22/0

27. S1. 2 , T $39 / 0$

28. S1. $2, \mathrm{U} 41 / 0$

29. S1. 1, L $68 / 4$

30. S1. $1, \mathrm{~S} 38 / 2$

31. S1. 1, E $25 / 4$ 

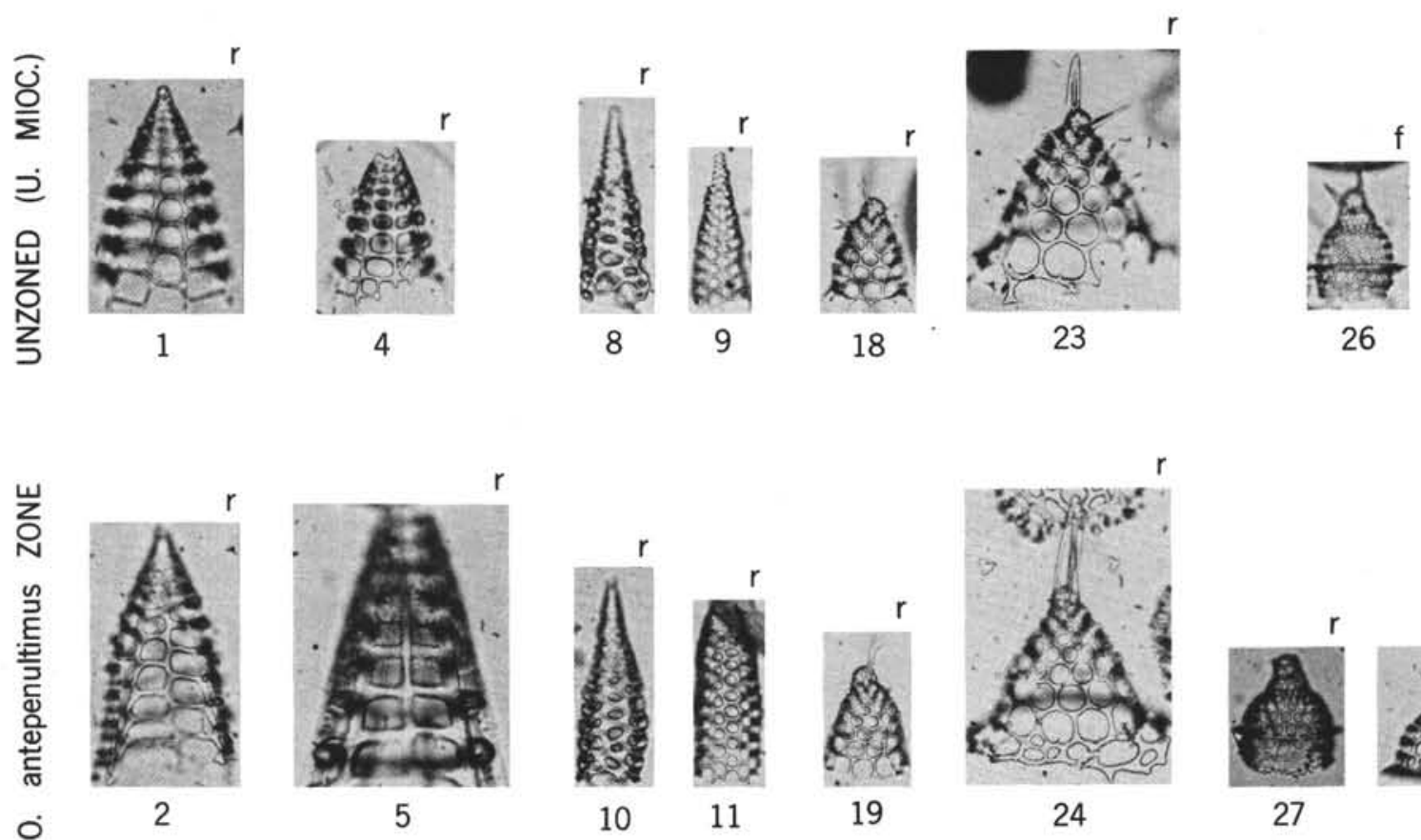

5
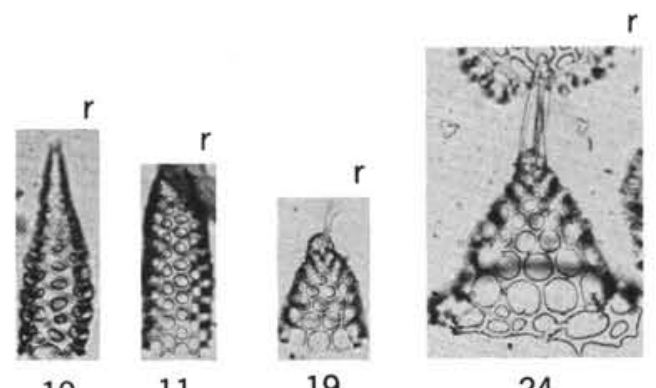

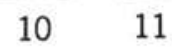

19

24

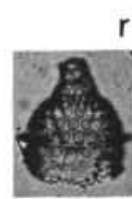

27

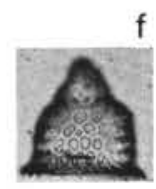

25

29
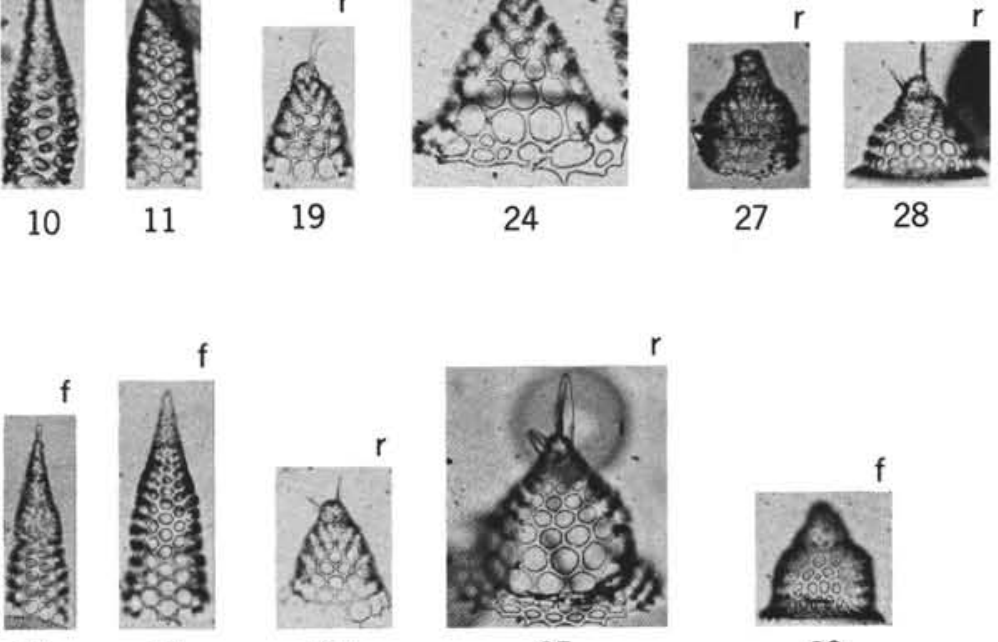

13

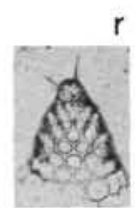

20
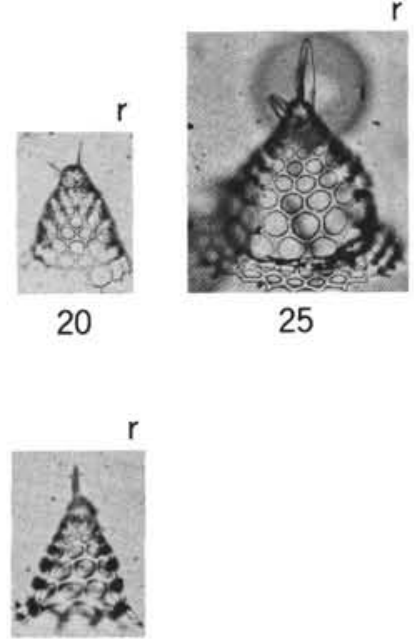

21

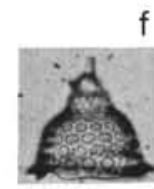

30

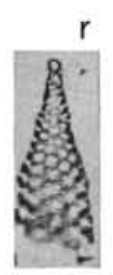

17

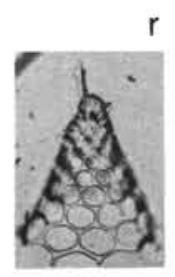

22 


\section{PLATE 10}

Figures 1-5 Stichopera pectinata group

1. S1. 2, B $62 / 0$

2. S1. $1, \mathrm{~K} 28 / 2$

3. S1. $1, \mathrm{G} 53 / 2$

4. S1. 1, B $19 / 4$

5. S1. 1, D 35/4

Figures 6-9 Pterocanium korotnevi(?)

6. S1. $1, \times 32 / 4$

7. S1. $1, \mathrm{M} 56 / 4$

8. S1. 1, P 39/4

9. S1. $1, \mathrm{~N} 32 / 2$

Figures 10-14 Lychnocanoma grande

10. S1. 2, L 32/4

11. S1. 1, V 39/1

12. S1. $1, \mathrm{~F} 47 / 0$

13. S1. $2,061 / 0$

14. S1. 2, R 32/1

Figures 15-17 Pterocanium(?) sp.

15. S1. $1, \mathrm{~S} 24 / 4$

16. S1. 2 , V $52 / 0$

17. S1. 1, Y $62 / 3$

Figures 18-20 Dictyophimus crisiae

18. S1. 1 , W $60 / 3$

19. S1. $1, \mathrm{R} 67 / 3$

20. S1. 2 , Y $52 / 2$

Figures 21-23 Eucyrtidium sp.cf. E. acuminatum

21. S1. $1, \mathrm{~S} 26 / 0$

22. S1. $1, \mathrm{~N} 22 / 1$

23. S1. $2, \mathrm{P} 23 / 0$

Figures 24-26 Lipmanella(?) sp.

24. S1. 1, Q 66/1

25. S1. 1, Q 70/1

26. S1. $2, \mathrm{U} 40 / 4$ 
PLATE 10

Ð
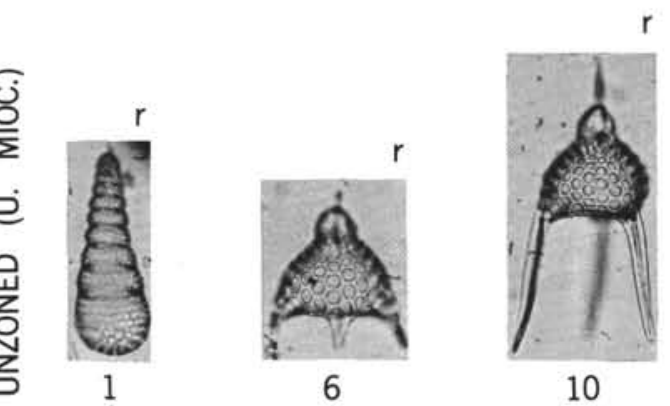

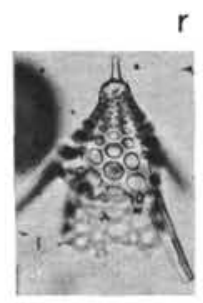

18

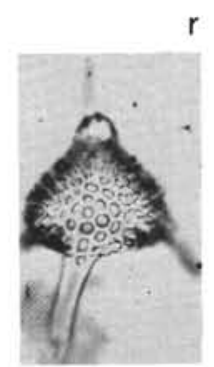

15

19

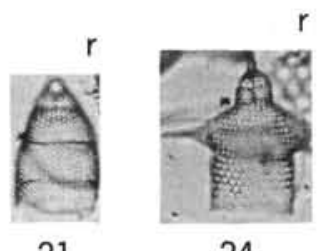

21

24

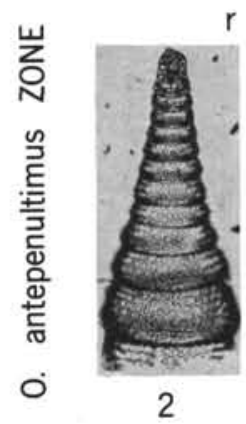

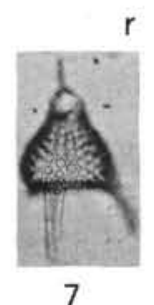

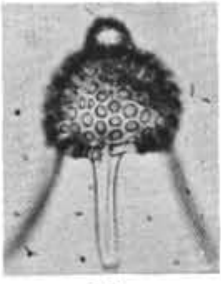

11

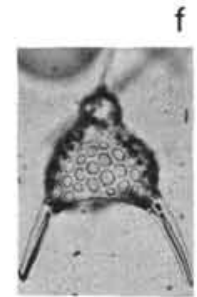

8

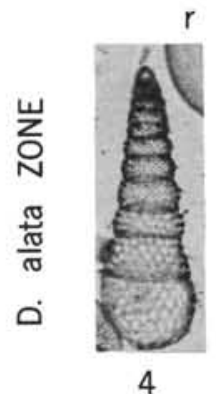

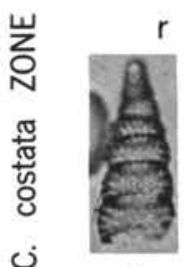

5

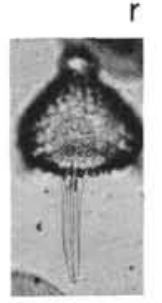

12

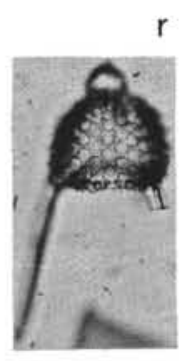

13

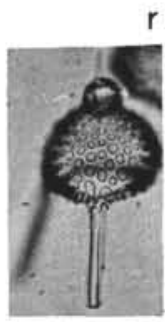

14

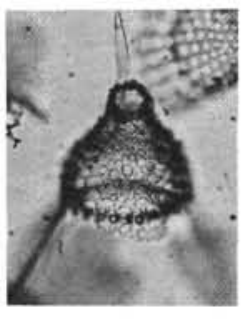

16

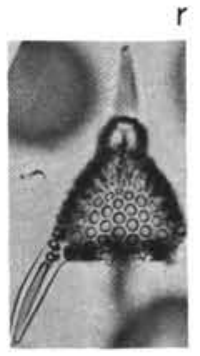

17
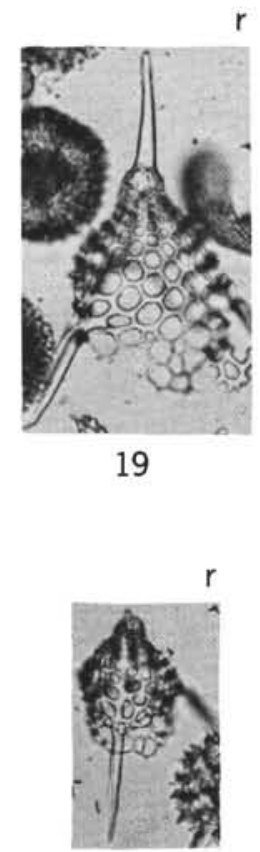

20

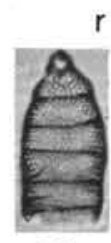

22

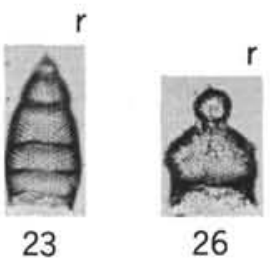


PLATE 11

Figures 1-5 Eucyrtidium calvertense

1. S1. 1, P 52/0

2. S1. $1, \mathrm{P} 68 / 2$

3. S1. $1, \mathrm{~J} 53 / 2$

4. S1. 2, Z 39/0

5. S1. 1, M $24 / 0$

Figure 6

Eucyrtidium inflatum(?)

S1. 2, S 26/2

Figure 7

Eucyrtidium inflatum

S1. 1, R 55/0

Figures 8-10

Stichocorys delmontensis

8. S1. $1, \mathrm{~K} 49 / 0$

9. S1. 1, L $31 / 4$

10. S1. $2, \mathrm{~S} 42 / 1$

Figure $11 \quad$ Stichcorys diploconus

S1. 2, G 61/0

Figures 12-15 Cyrtocapsella tetrapera

12. S1. 1, Q 38/4

13. S1. 2, C $23 / 1$

14. S1. 1, J 20/0

15. S1. $1, \mathrm{U} 62 / 2$

Figures 16-18 Cyrtocapsella cornuta

16. S1. 2, W $33 / 2$

17. S1. 1, D $62 / 0$

18. S1. 2, K $29 / 1$

Figures 19,20 Cyrtocapsella japonica

19. S1. 2, X $60 / 0$

20. S1. 1, M 39/1

Figures $2122 \quad$ Lithopera bacca

21. S1. 1, M 68/4

22. S1. 1, J $35 / 1$

Figure 23 Lithopera neotera

S1. 1, U 20/0

Figure $24 \quad$ Lithopera thornburgi

S1. 1, H 66/0

Figure 25 Lithopera renzae

S1. 1, P 20/2

Figures 26-28 Theocorys redondoensis

26. S1. 2, X 61/0

27. S1. $2, \mathrm{~J} 46 / 2$

28. S1. 2, Y $64 / 0$

Figure 29 Stichocorys peregrina

S1. 1, F 30/2 
PLATE II
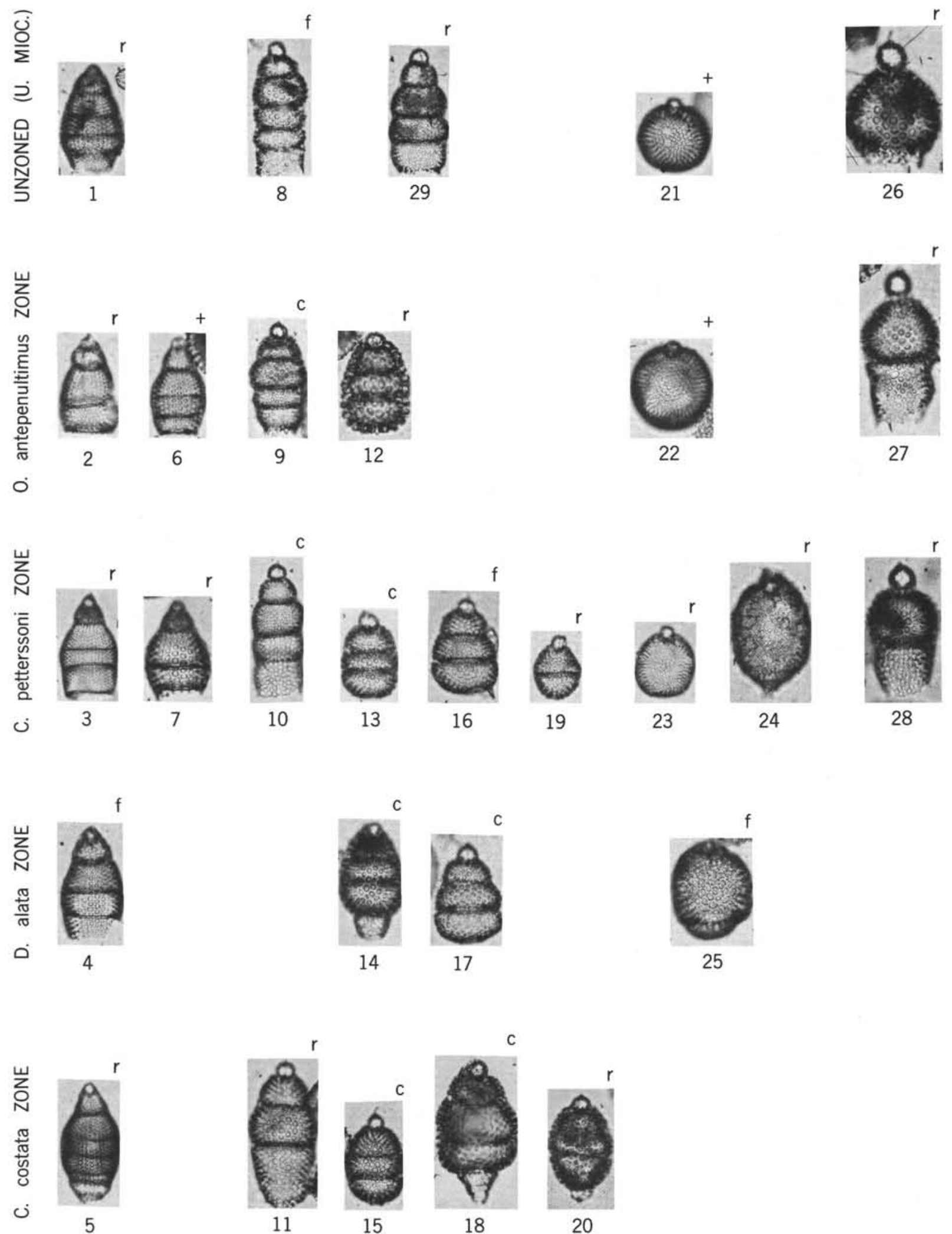

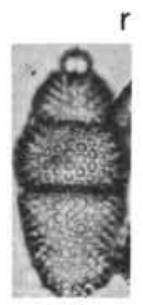

11

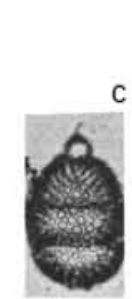

15
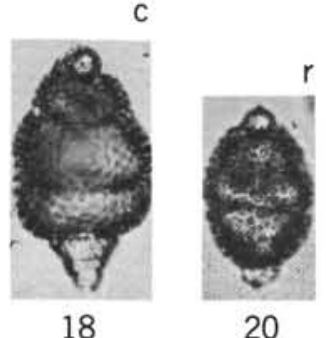
PLATE 12

Figures 1-6 Carpocaniidae, gen. et spp. indet.

1. S1. $1, \mathrm{~N} 25 / 1$

2. S1. 2 , W $41 / 1$

3. S1. $1, \mathrm{H} 34 / 4$

4. S1. $2, \mathrm{Y} 61 / 3$

5. S1. 2, P. $26 / 4$

6. S1. 2, M 30/3

Figures 7-9 Theoconus zancleus(?)

7. S1. $1, \mathrm{~N} 27 / 0$

8. S1. $1, \mathrm{H} 65 / 1$

9. S1. $2, \mathrm{~S} 64 / 2$

Figures 10-14 Lamprocyrtis(?) hannai

10. S1. 1, D $41 / 2$

11. S1. $1, \mathrm{~F} 66 / 4$

12. S1. 2, S $25 / 3$

13. S1. $2, \mathrm{~V} 65 / 2$

14. S1. 1, R $53 / 0$

Figures 15-18 Sethocorys spp

15. S1. 1, T 39/1

16. S1. $1, \mathrm{~S} 22 / 2$

17. S1. 1, Q 23/1

18. S1. 1, Z 5/1, L

Figures 19, 20 Lithomitra lineata group

19. S1. $1, \mathrm{~V} 44 / 0$

20. S1. $1,040 / 0$

Figures 21-23 Siphocampe corbula(?)

21. S1. 1 , D $41 / 4$

22. S1. 1, X $28 / 3$

23. S1. 1 , R $53 / 0$

Figures 24-27 Artostrobium auritum group

24. S1. 1, S $20 / 0$

25. S1. 2, G 53/4

26. S1. 1, L 23/1

27. S1. 1, E $55 / 0$

Figures 28-31 Artostrobium miralestense

28. S1. 1, F 22/1

29. S1. 2 , G $58 / 0$

30. S1. $2, X 71 / 0$

31. S1. 2 , C $20 / 2$

Figure $32 \quad$ Calocycletta sp.

S1. 1, X 20/2 
PLATE 12

흘

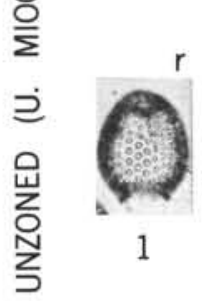

닛

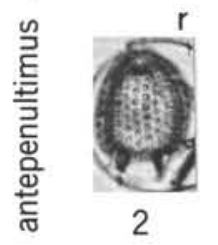

$\circ$
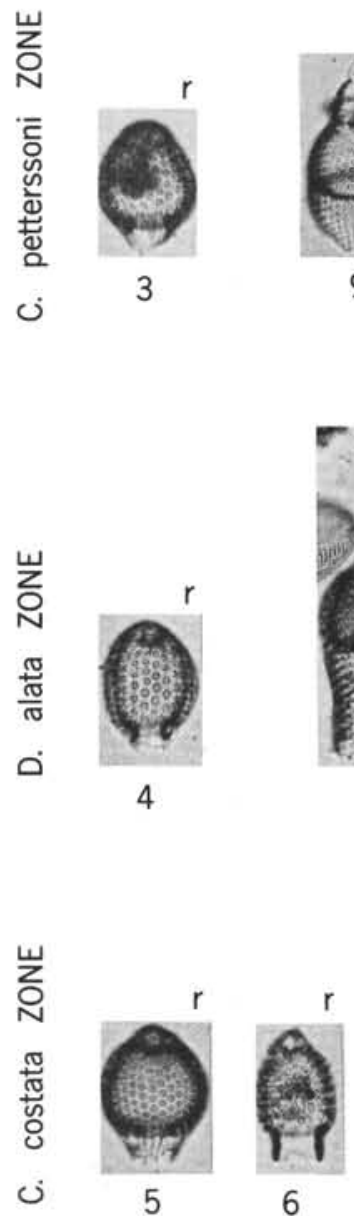
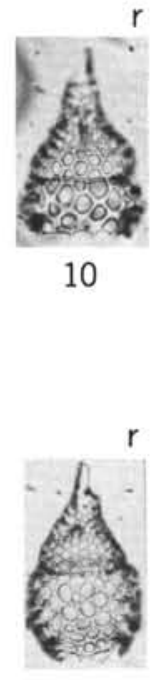

11

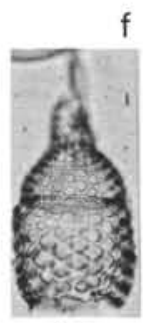

12

9
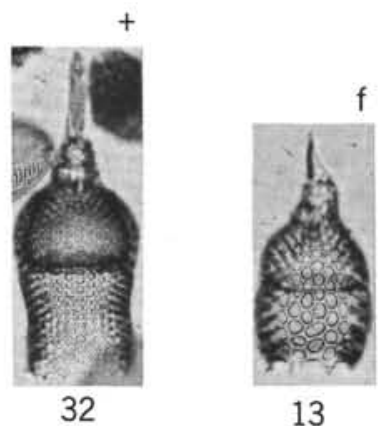

13

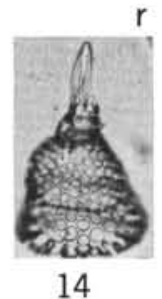

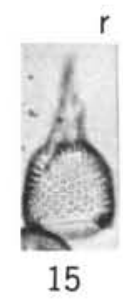
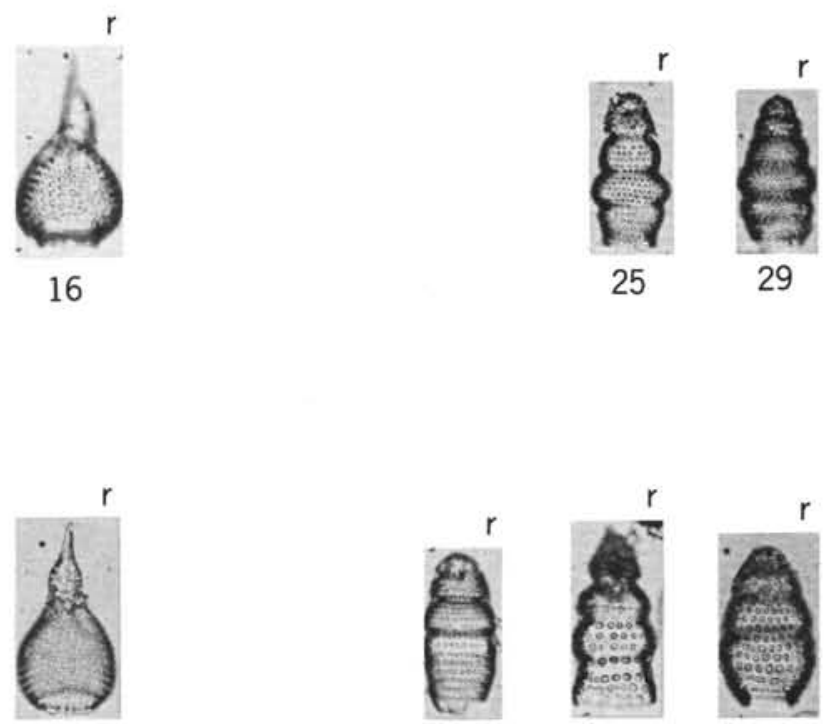

17
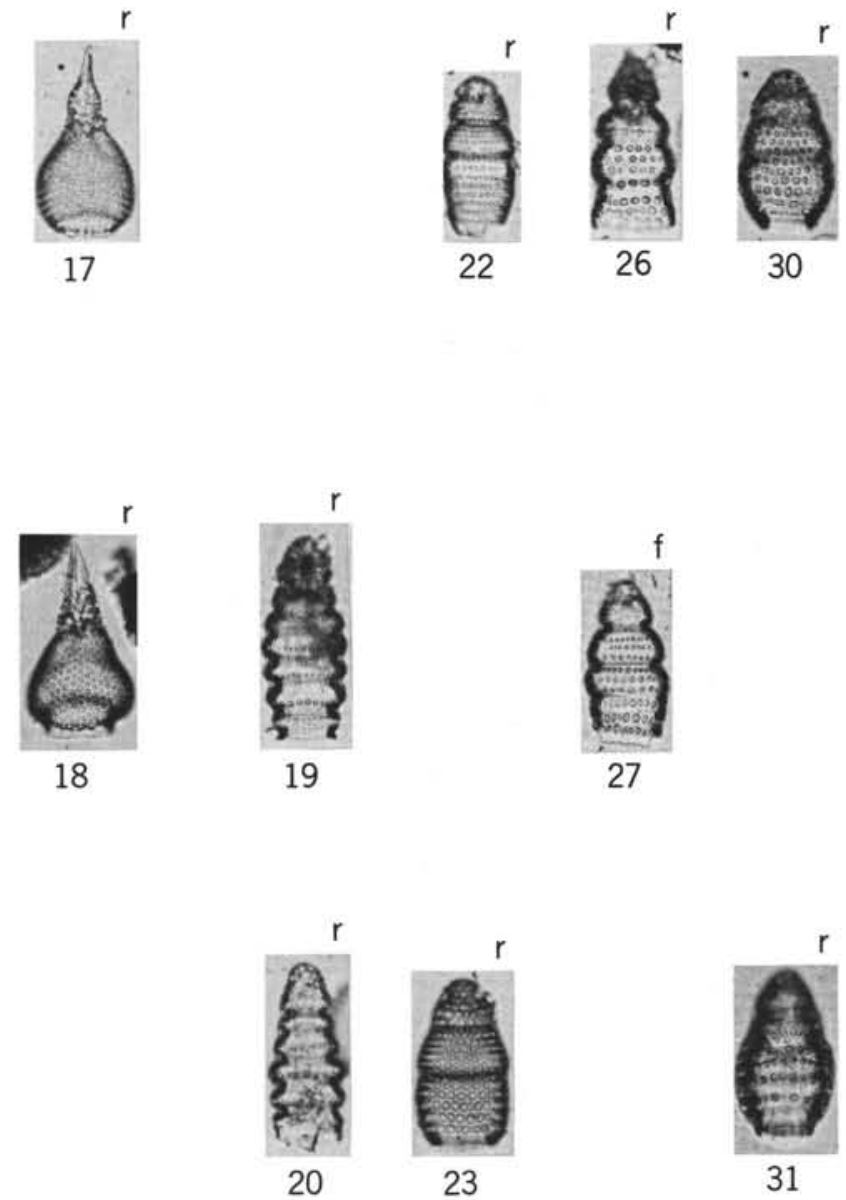


\section{PLATE 13}

Figures 1-5 Sphaeropyle robusta

1,2,3. Paratype, 177A-6-4 (70-72), S1 . 1, T 70/0

4,5. Holotype, 173-16-1 (70-72), S1. 1, T 35/4

Figures 6-8 Sphaeropyle langii

173-6-2 (55-57), S1. 2, $041 / 0$

Figures 9,10 Stichocorys peregrina

9. 173-14-3 (57-59), S1. 2, K 70/1

10. 173-14 3(57-59), S1. 1, G 60/3

Figure 11 Stichocorys armata

173-28-3 (57-59), S1. 1, O 47/2

Figure 12 Stichocorys diploconus

173-31-1 (128-120), S1. 2, J 65/3 
PLATE 13

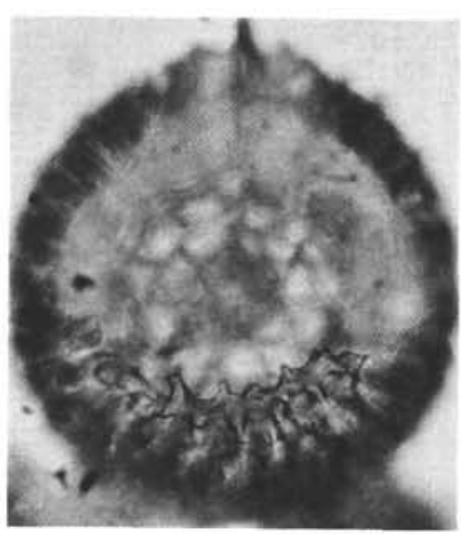

1

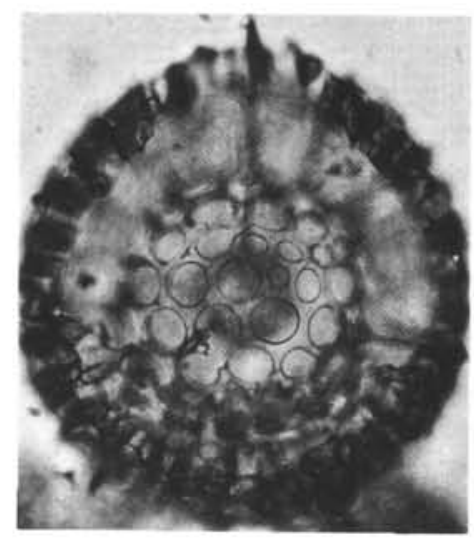

2

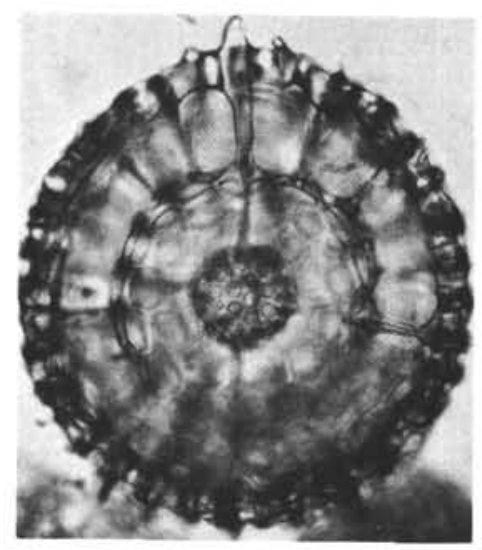

3
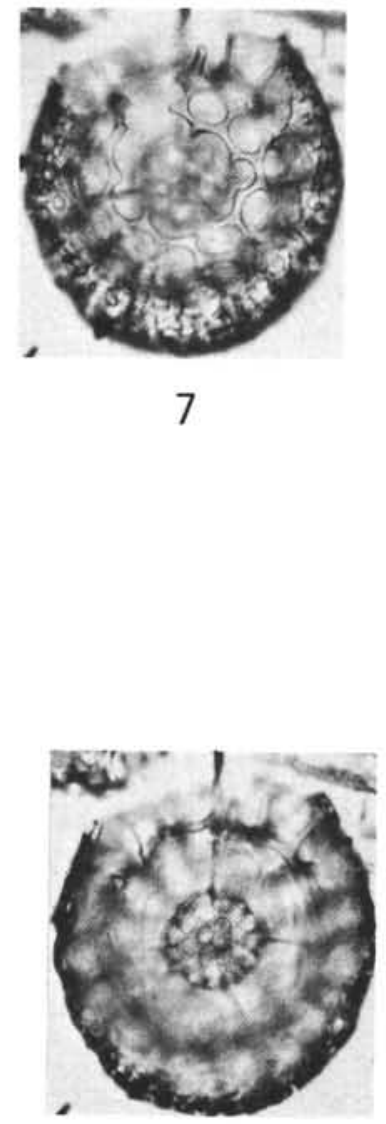

8

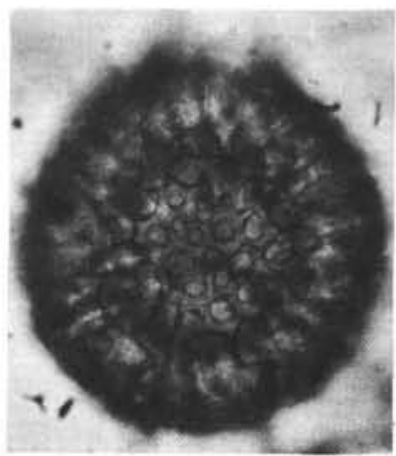

4

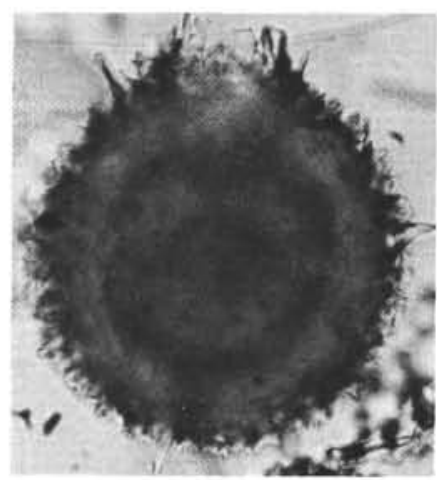

5

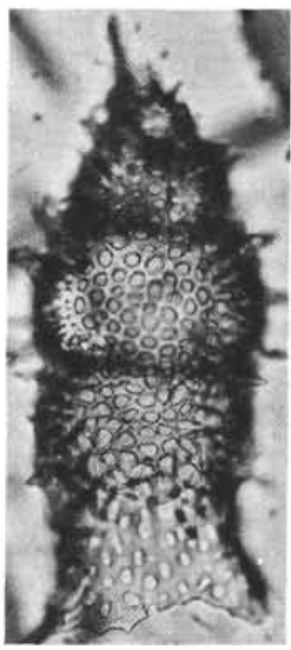

11

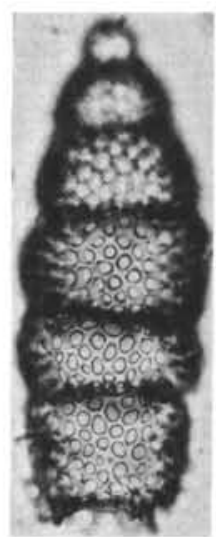

10

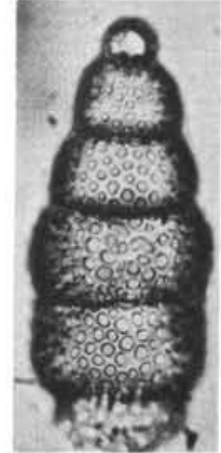

9

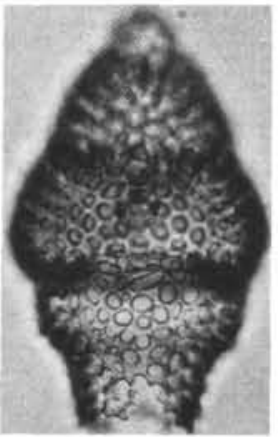

12 
PLATE 14

Figures 1-4 Stylacontarium acquilonium

1,2. 173-11-3 (57-59), S1. 1, T 32/0

3,4. 173-9-3 (57-59), S1, 2, L 35/0

Figures 5-8 Stylacontarium sp. aff. S. bispiculum 5,6. 173-15-3 (57-59), S1. 2, J 51/4

7,8. 173-19-4 (57-59), S1. 2, N 68/4 
PLATE 14

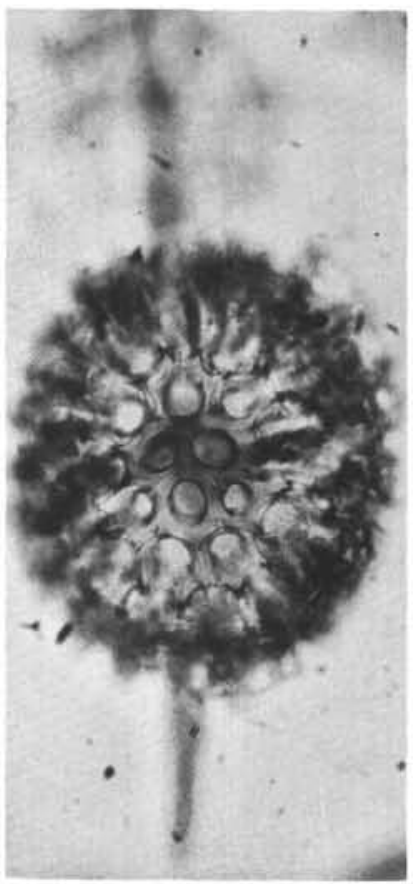

1

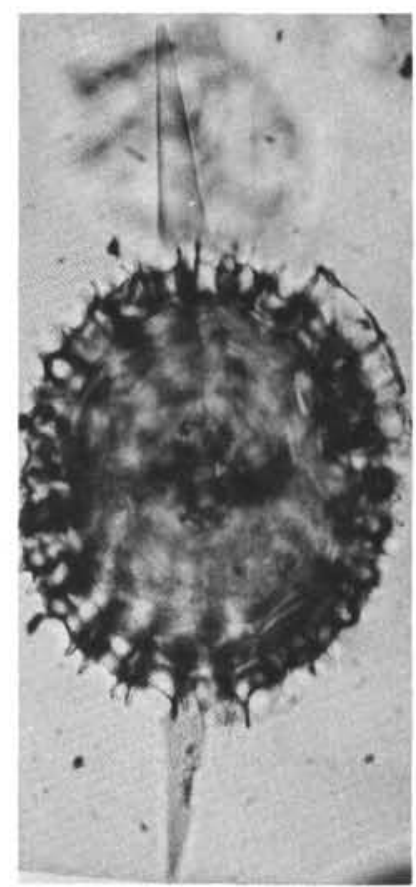

2

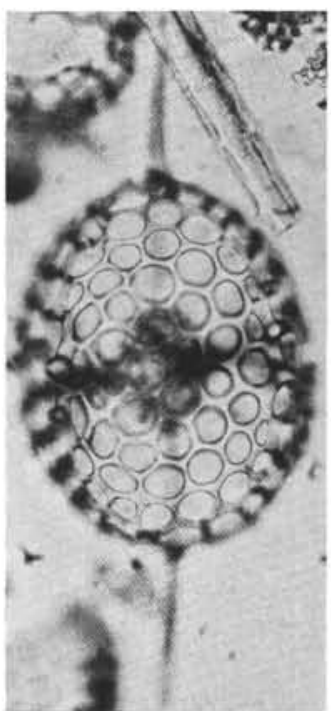

3

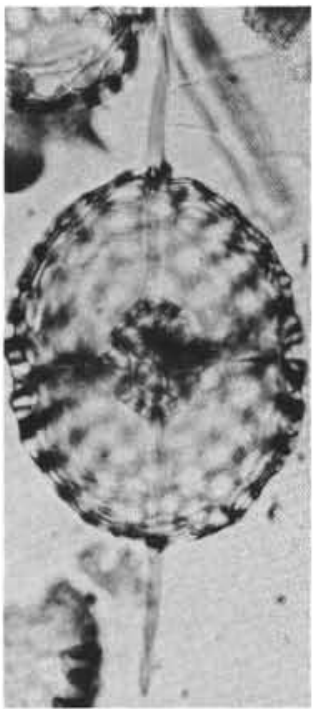

4

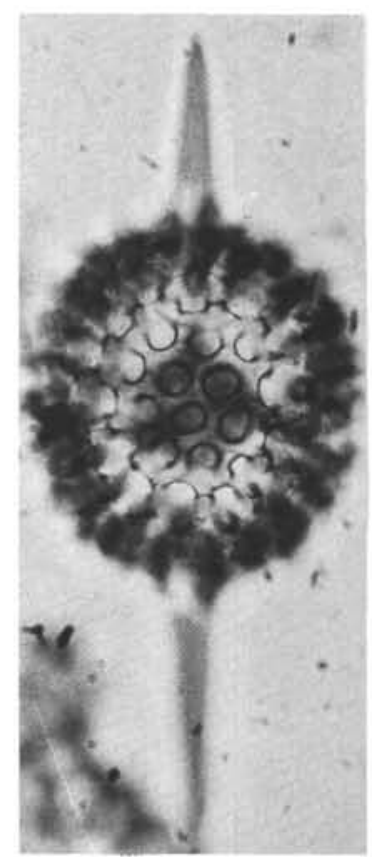

5

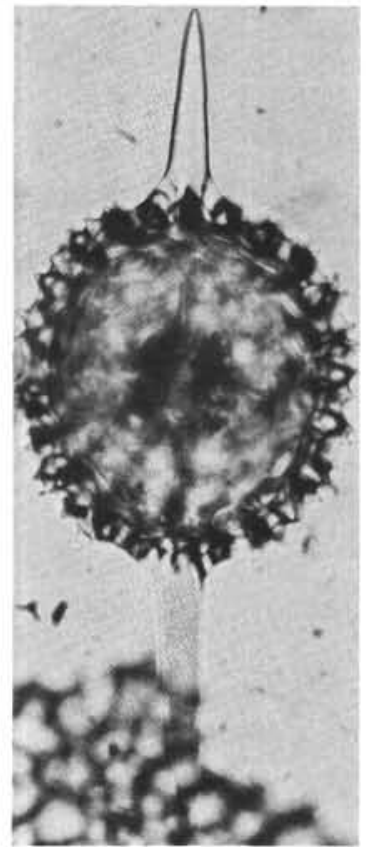

6

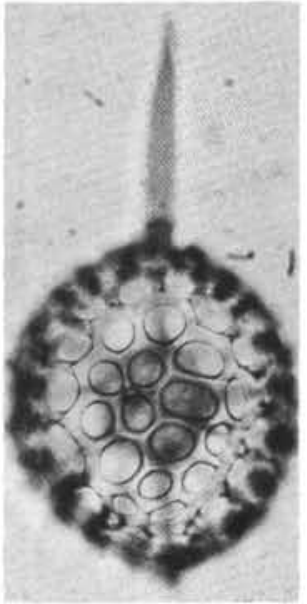

7

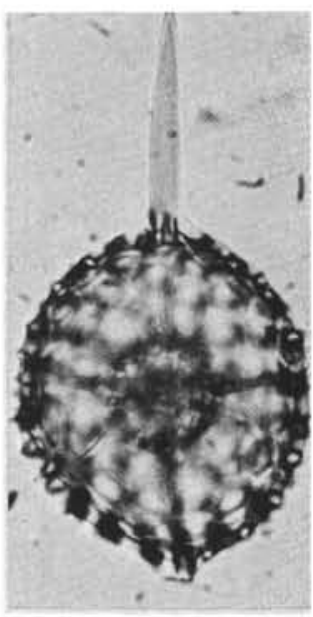

8 


\section{PLATE 15}

Figures 1-3 Lamprocyrtis haysi

1. Holotype, 173-2-2 (45-47), S1. 1, S 29/2

2. 173-2-5 (65-67), S1. 1, D 85/2

3. S.I.O., Core DWBG $12,6-8 \mathrm{~cm}, \mathrm{~S} 1$. 1, F $58 / 3$

Figures 4,5 Lamprocyrtis neoheteroporos

4. 173-5-5 (57-59), S1. 1, F 25/2

5. Holotype, 173-5-3 (57-59), S1. 1, X 34/2

Figure 6 Lamprocyrtis heteroporos

6. 173-14-2 (92-94), S1. 1, M 29/2

Figures 7-10 Eucyrtidium inflatum

7,8. Holotype, 173-22-2 (57-59), S1. 1, T 45/3

9,10. 173-25-5 (50-52), S1. 1, W 69/0

Figures 11-14 Stylacontarium bispiculum

11,12. S.I.O. Core DWBG $68,7-9 \mathrm{~cm}$, S1. 1 , D $38 / 4$

13,14. S.I.O. Core DWBG 68, 7-9 cm, S1. 2, Z 42/2 
PLATE 15

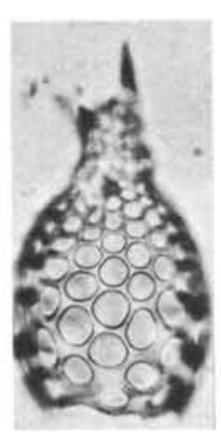

1

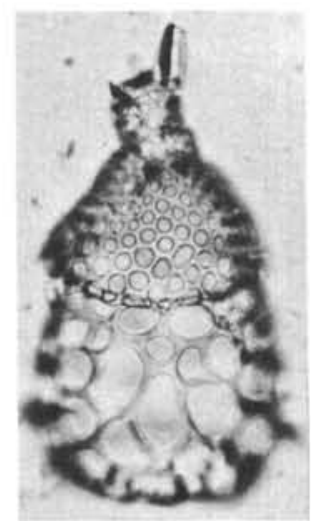

6

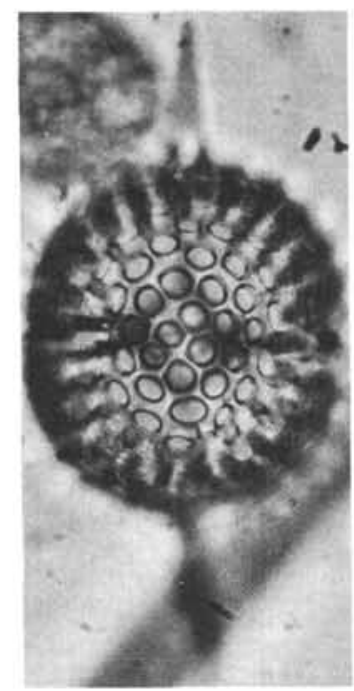

11

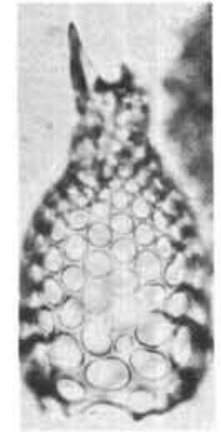

2

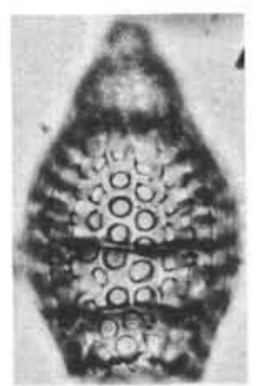

7

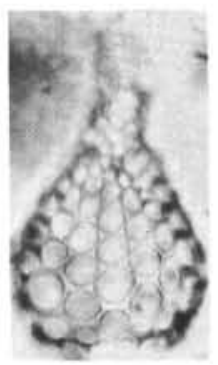

3

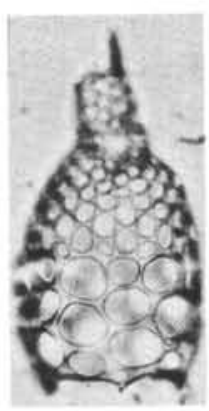

4

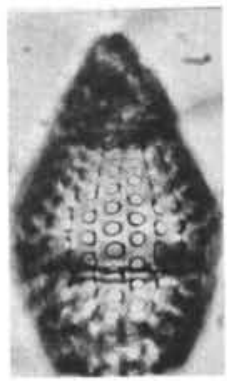

9

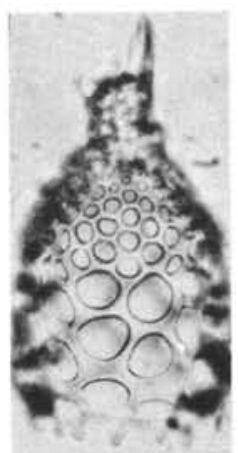

5

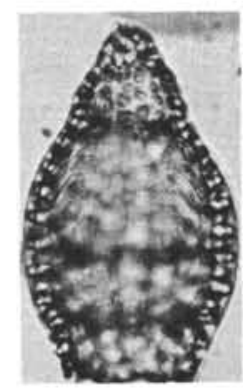

8

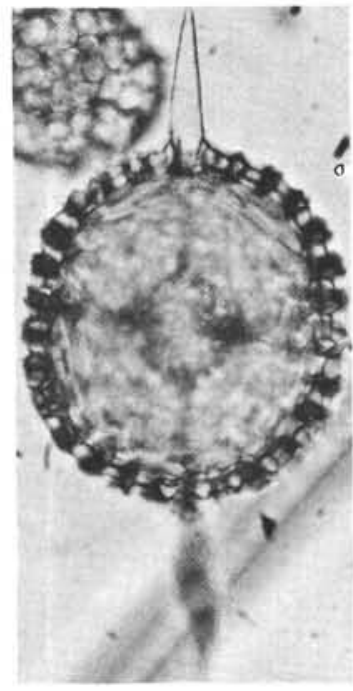

12

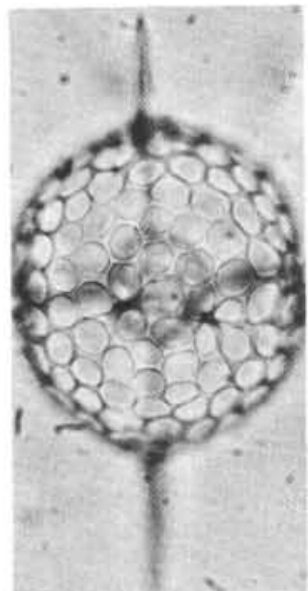

13

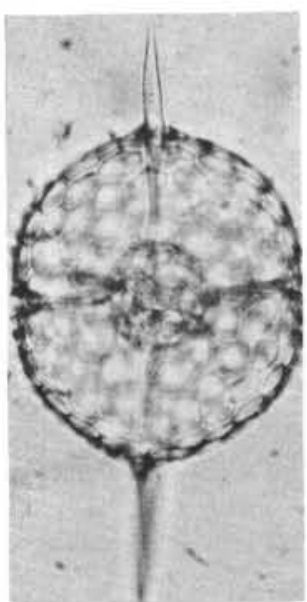

14

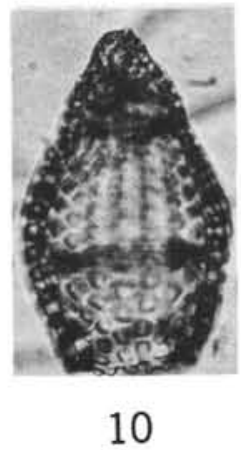

